\title{
CLIMATIC DATA FOR MIRROR LAKE, WEST THORNTON, NEW HAMPSHIRE 1984
}

By

A. M. Sturrock, D. C. Buso, J. L. Scarborough, T. C. Winter

U.S. GEOLOGICAL SURVEY

Open-File Report $\quad 86-134$

Prepared in cooperation with

CORNELL UNIVERSITY and the

INSTITUTE OF ECOSYSTEM STUDIES,

THE NEW YORK BOTANICAL GARDEN

Lakewood, Colorado 1986 
UNITED STATES DEPARTMENT OF THE INTERIOR

DONALD PAUL HODEL, Secretary

GEOLOGICAL SURVEY

Dallas L. Peck, Director

For additional information write to:

Thomas C. Winter U.S. Geological Survey

Box 25046, MS 413

Denver Federal Center

Lakewood, CO 80225
Copies of this report can be purchased from:

Open-File Services Section Western Distribution Branch U.S. Geological Survey Box 25425, MS 306 Denver Federal Center Lakewood, CO 80225

Telephone: (303)236-7476 
CONTENTS

Page

Abstract-1

Introduction-10

Data collection and presentation-

Acknowledgments -

Reference-1-

TABLES

Page

Table 1. Summary of 1984 energy-budget data: Raft station--(1) daily average lake-surface water temperature, (2) daily average dry- and wet-bulb air temperatures, (3) daily average vapor pressure at water surface and 2 meters above water surface, (4) Bowen ratio, and (5) daily average wind speed at three levels above the lake surface; Land station--daily shortand 1ong-wave radiation-1

2. Summary of 1984 data: Raft-station temperature--(1) daily average lake-surface water temperature, (2) daily average dry-bulb air temperature, (3) daily maximum and minimum dry-bulb air temperatures and the time they occurred, and

(4) daily average wet-bulb air temperature-1...

3. Summary of 1984 data: Raft-station wind speed--(1) daily average wind speed at 1,2 , and 3 meters above lake surface, (2) daily maximum and minimum wind speed at the level and

the time they occurred-1...

4. Summary of 1984 data: Land-station radiation--(1) daily total short-wave solar radiation, (2) daily maximum short-wave solar radiation and the time it occurred, (3) daily total long-wave atmospheric radiation, and (4) daily maximum and minimum long-wave radiation and the time they occurred--.-- 


\section{METRIC CONVERSION FACTORS}

Multiply

meter

kilometer

centimeter

millibar

millibar

mile per hour

calories per square centimeter

per minute

calories per square centimeter

per day

calories per square centimeter

per day
$B y$

3.281

0.621

0.394

0.0145

1.0197

1.609

$1.433 \times 10^{-3}$

278.96

25.913

To obtain

feet

mile

inch

pounds per square inch

grams per square centimeter

kilometer per hour

watts per square meter

watts per square meter

watts per square foot

To convert degrees Celsius $\left({ }^{\circ} \mathrm{C}\right)$ to degrees Fahrenheit $\left({ }^{\circ} \mathrm{F}\right)$ use the following formula: $\left({ }^{\circ} \mathrm{C} \times 9 / 5\right)+32={ }^{\circ} \mathrm{F}$. 
CLIMATIC DATA FOR MIRROR LAKE, WEST THORNTON, NEW HAMPSHIRE, 1984

By A. M Sturrock, D. C. Buso,

J. L. Scarborough, and T. C. Winter

\begin{abstract}
Research on the hydrology of Mirror Lake, West Thornton, New Hampshire, includes study of evaporation. Presented here are those climatic data needed for energy-budget and mass-transfer evaporation studies, including: watersurface temperature, dry-bulb and wet-bulb air temperatures, vapor pressure at and above the water surface, wind speed, and short- and long-wave radiation. Data are collected at raft and land stations.
\end{abstract}

\title{
INTRODUCTION
}

Climatic data are being collected at Mirror Lake, West Thornton, New Hampshire, as part of a continuing study of the hydrology of the lake by the U.S. Geological Survey, Cornell University, and the Institute of Ecosystem Studies, The New York Botanical Garden. Mirror Lake is one of several lakes in different parts of the United States that have been selected for intensive study of hydrological and related chemical and biological processes. The rationale for selection of Mirror Lake is given by Winter (1984).

\section{DATA COLLECTION AND PRESENTATION}

Data presented here are being collected principally for studies of evaporation; therefore, data are collected only during the time the lake is ice-free. Data for 1984 were collected from April 23 (Julian day 114) to November 30 (Julian day 335). Within each table of data, the data are grouped according to energy-budget periods; the periods are defined by the dates thermal surveys were made in the lake. For example, the first energy-budget period for 1984 is Julian days 114 through 127.

Climatic instruments are located on a raft near the middle of the lake and at a land station. Instruments on the raft include anemometers at 1,2 , and 3 meters above the water surface, a thermistor psychrometer with dry- and wet-bulb temperature sensors fixed at 2 meters above the water surface, and a water-temperature sensor located beneath the raft at a depth of about 1 centimeter. Data from the above sensors are recorded by a digital data logger located on the raft. The data logger scans the sensors every minute and calculates hourly and daily averages and totals. In addition, maximum and 
minimum values and the times they occur are saved and recorded at midnight of each day for selected sensors. Additional analog instruments for measuring water-surface temperature and wind speed also are located on the raft. These are used to backup the primary instruments for quality control and for filling in missing data.

The land station consists of short- and long-wave radiometers, located at the U.S. Forest Service Hubbard Brook Station, about 0.4 kilometer west of Mirror Lake. These data also are recorded by a digital-data logger that operates similarly to the one on the raft. A backup hygrothermograph that records air temperature and relative humidity is located on the shore of Mirror Lake. Calibration checks with laboratory-quality thermometers and motorized psychrometers are made weekly. Vapor pressure of water $\left(e_{0}\right)$ is calculated using water-temperature data and assumes the air is completely saturated at the air-water interface.

Data presented here are daily summaries. For periods during which the primary instruments were not operating properly, daily values were obtained by regression using data from backup instruments, provided a satisfactory statistical relationship could be established. Data used to establish regressions were selected so they bracketed the period of missing or inadequate data. Only table 1 , which is considered to be the primary source of data for evaporation studies, includes values obtained by regression.

Although only daily values are reported here, hourly values also were recorded. Hourly values are voluminous and expensive to reproduce, but they are available for all or part of the period of record, on request to $T$. C. Winter.

\section{ACKNOWLEDGMENTS}

We are grateful to Robert Pierce and Wayne Martin of the U.S. Forest Service for allowing us to place the land station at Hubbard Brook Experimental Forest Headquarters. We also thank Polly Ann Frost for permission to place the hygrothermograph on her property. Partial funding for this study is from a National Science Foundation grant to G. E. Likens (Institute of Ecosystem Studies, The New York Botanical Garden) and F. H. Bormann (Yale University).

\section{REFERENCE}

Winter, T. C., 1984, Geohydrologic setting of Mirror Lake, West Thornton, New Hampshire: U.S. Geological Survey Water-Resources Investigations Report $84-4266,61 \mathrm{p}$. 

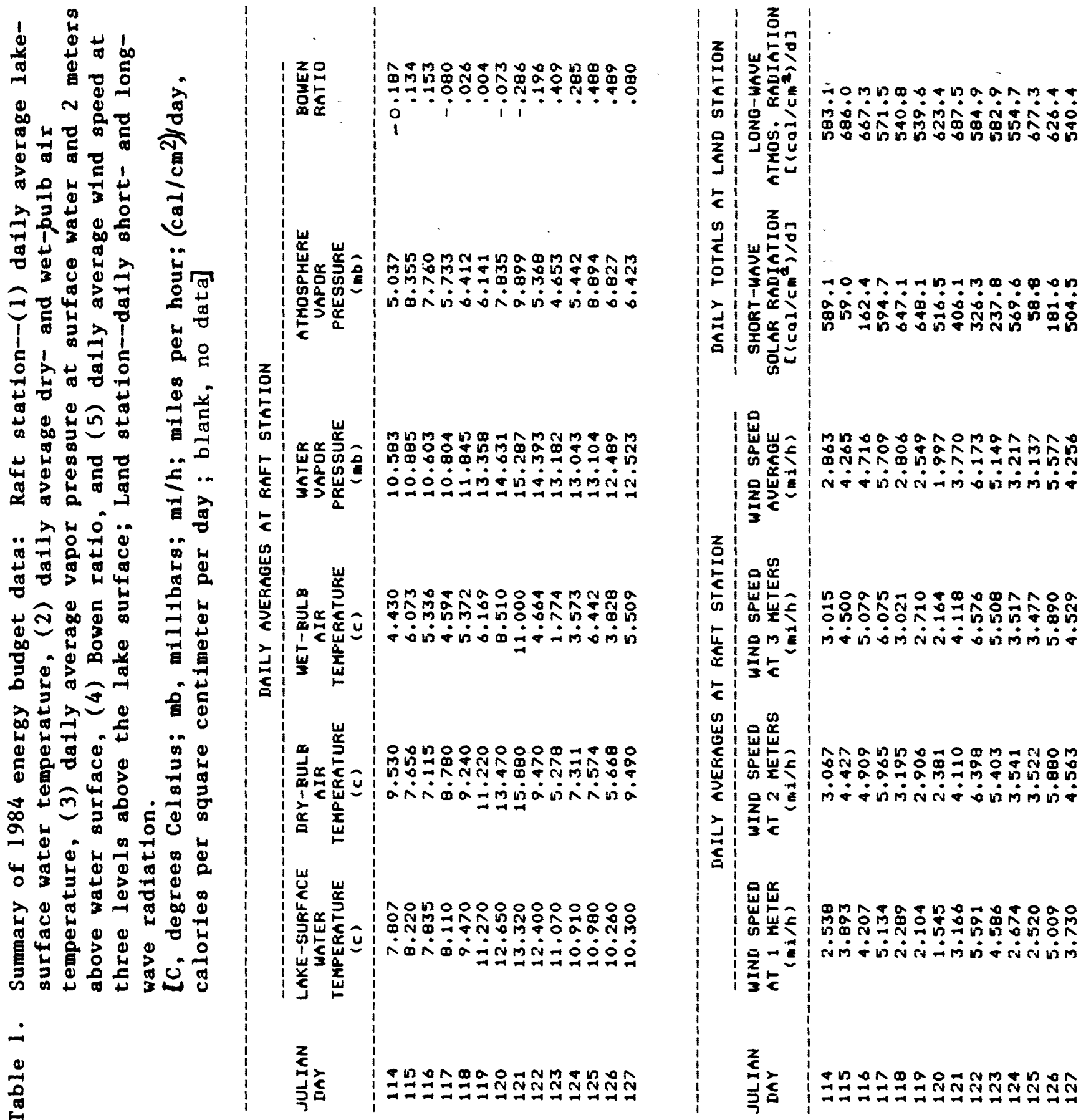

(3) 

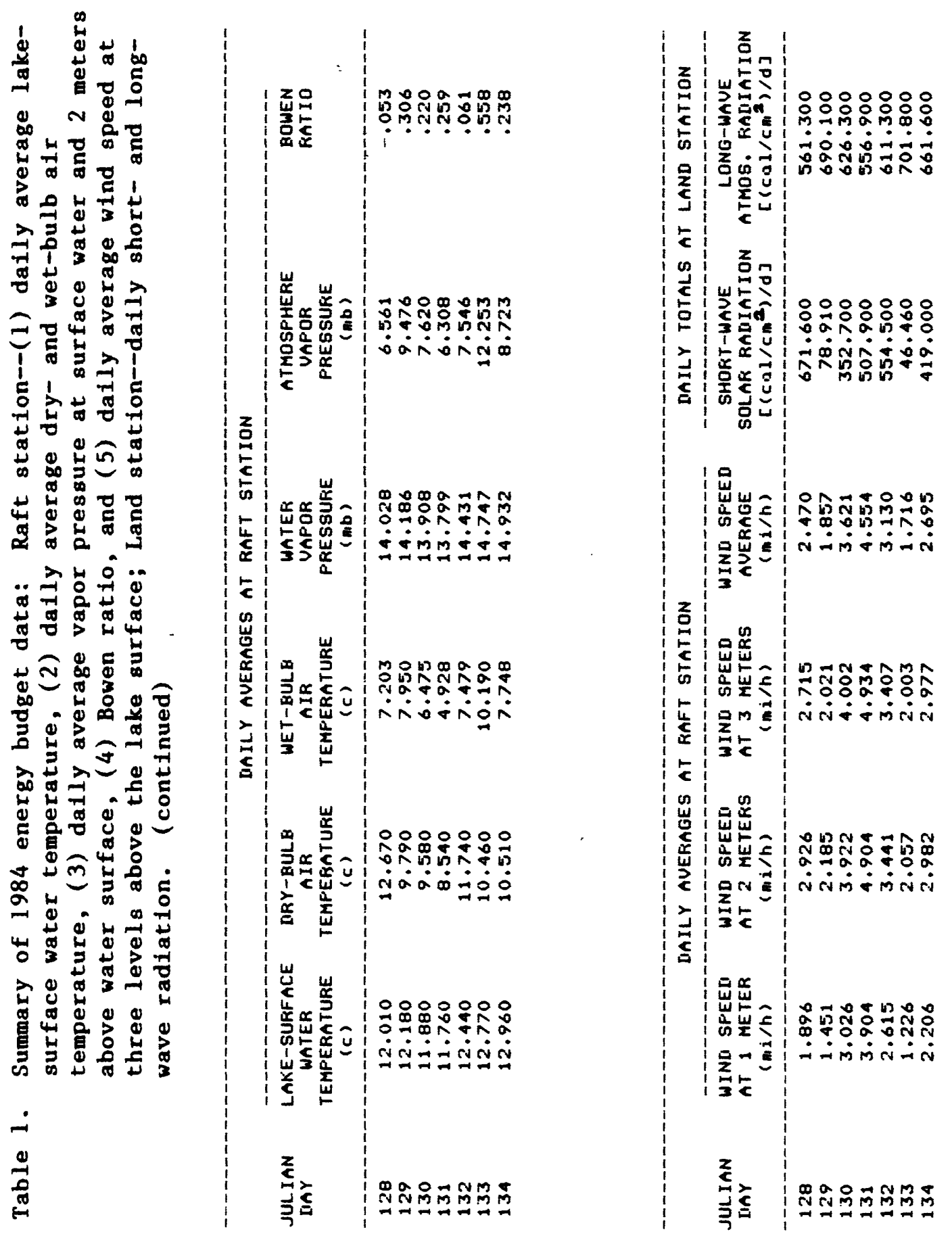

(4) 


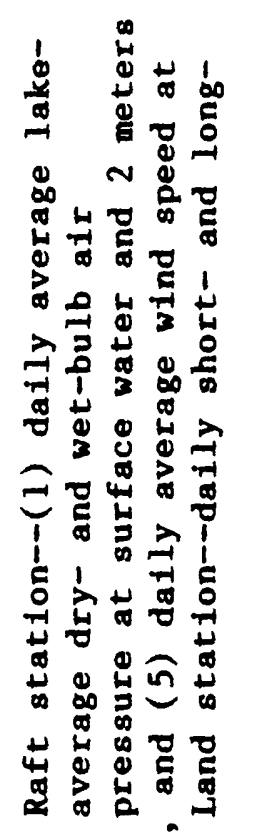

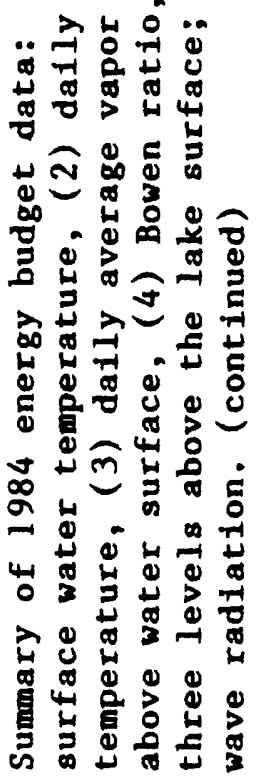

車

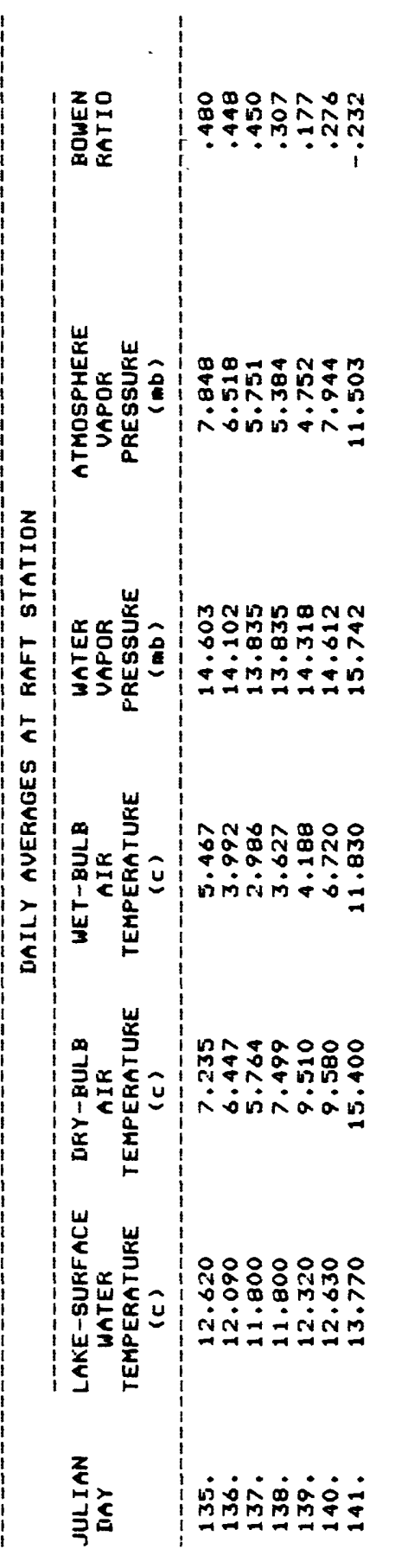

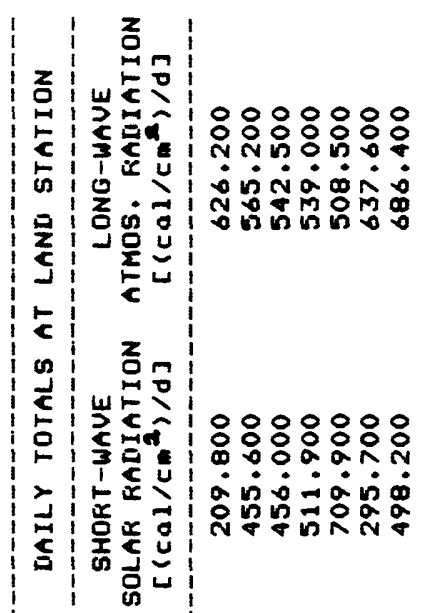
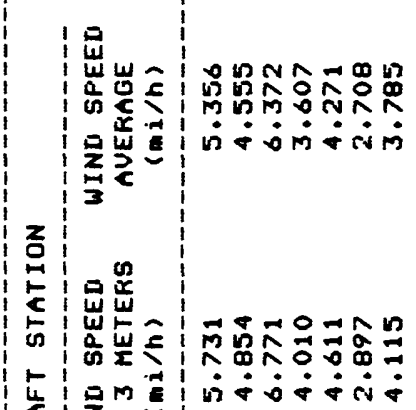

占可专

(c)

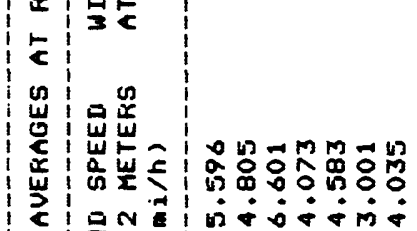

C UN

c

娄

c

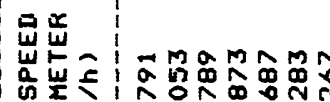

|ि-

帘舟

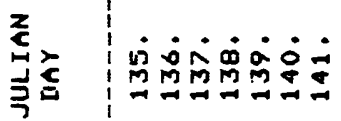



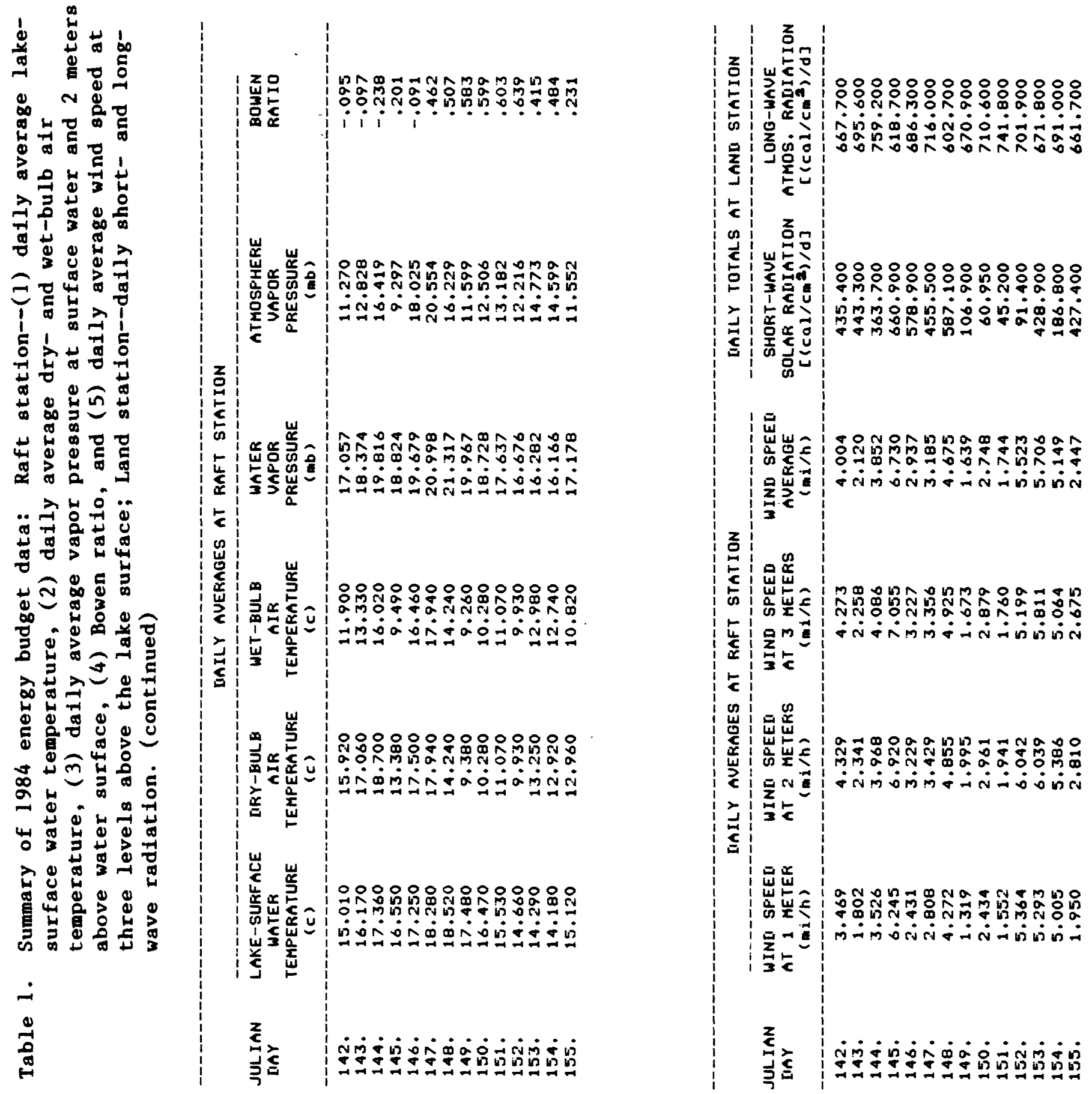

(6) 

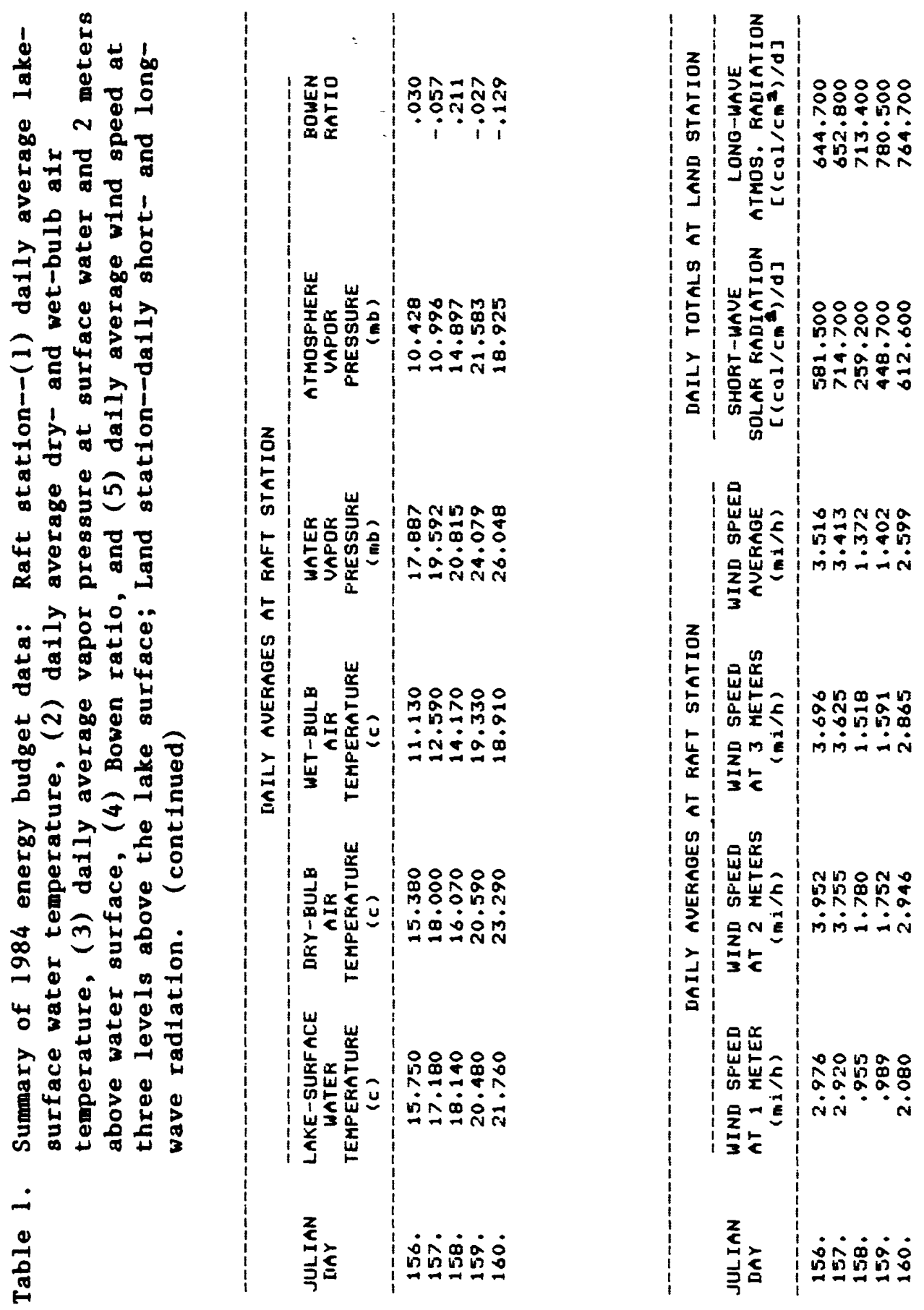

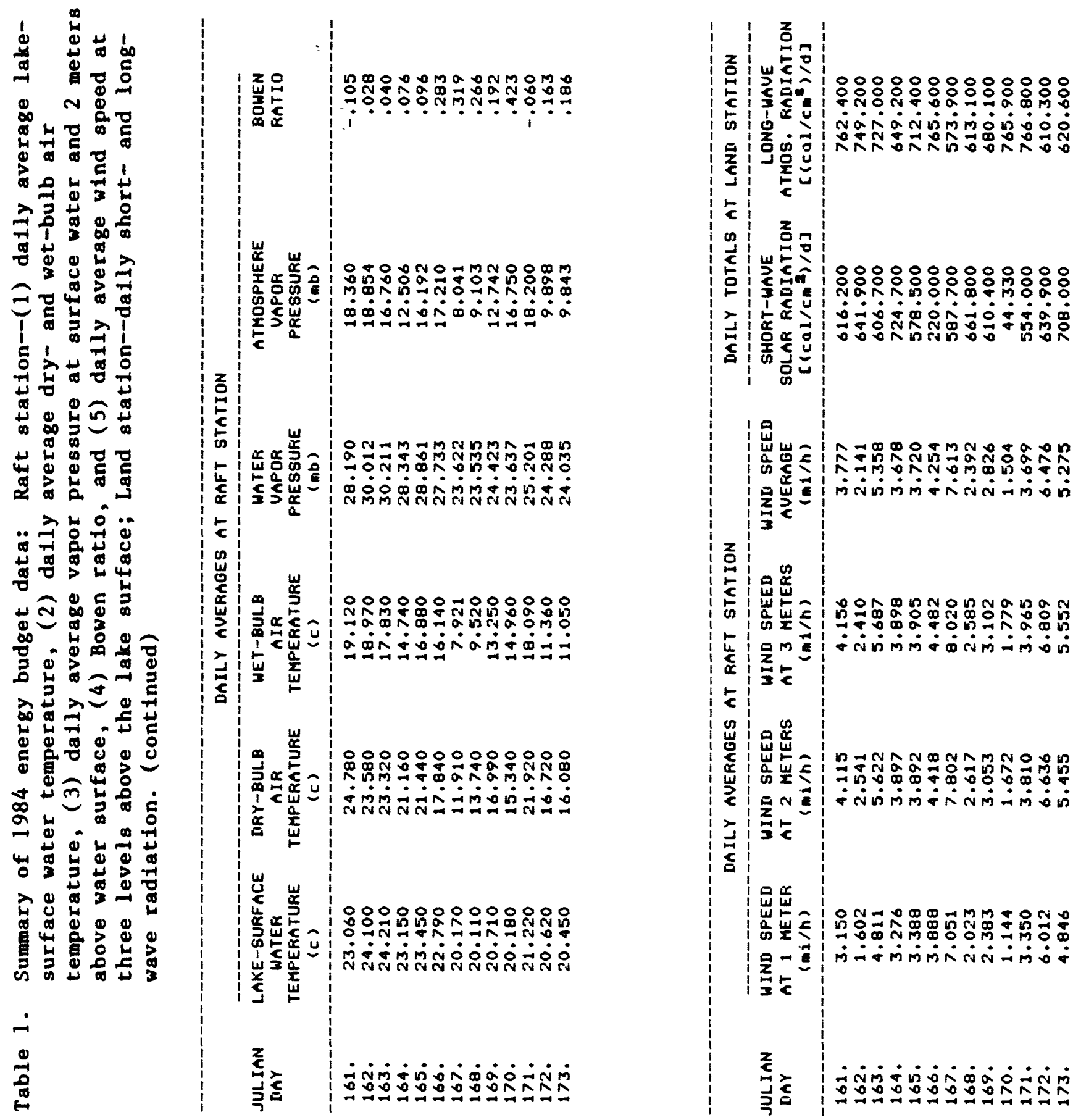

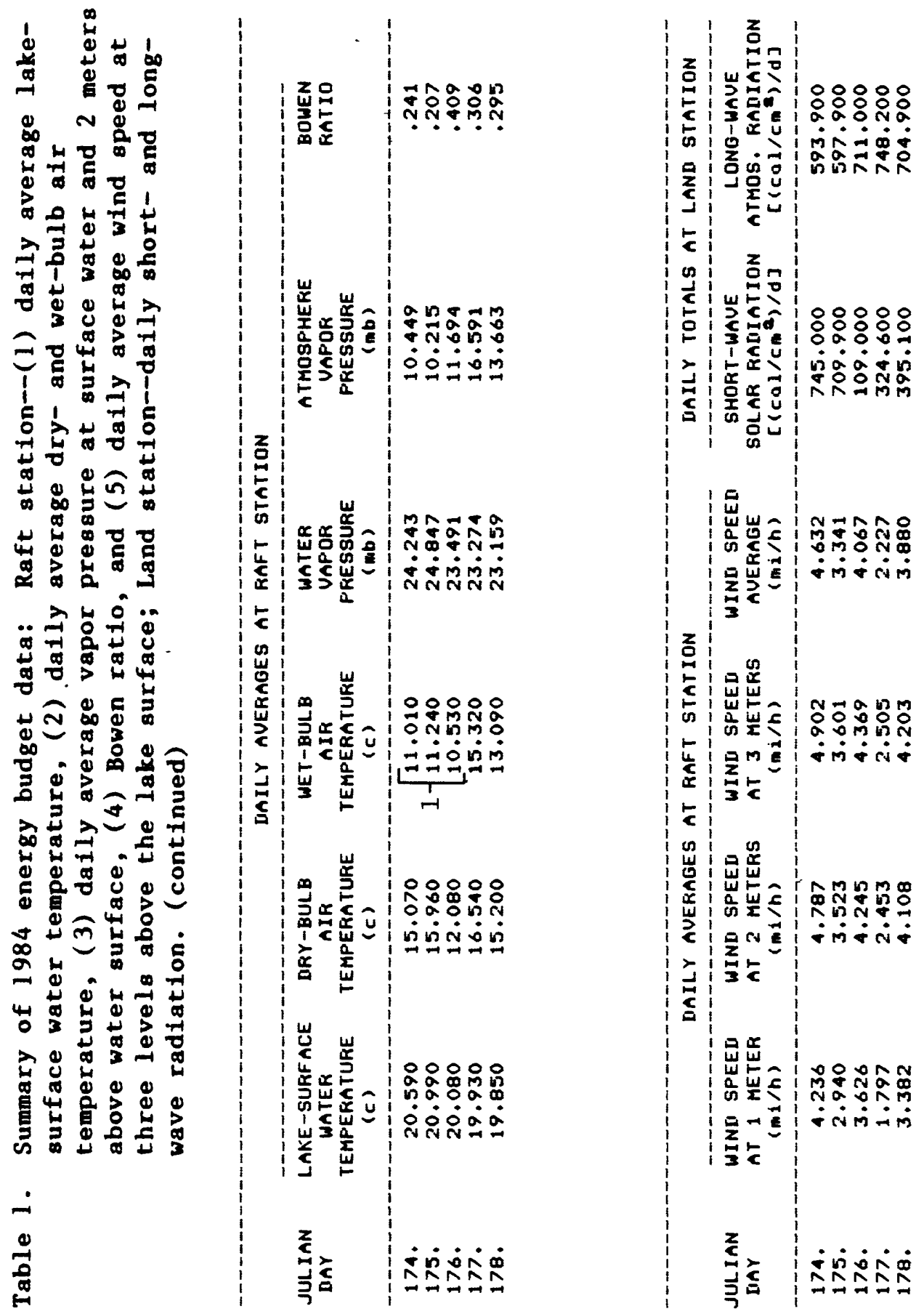

(9) 


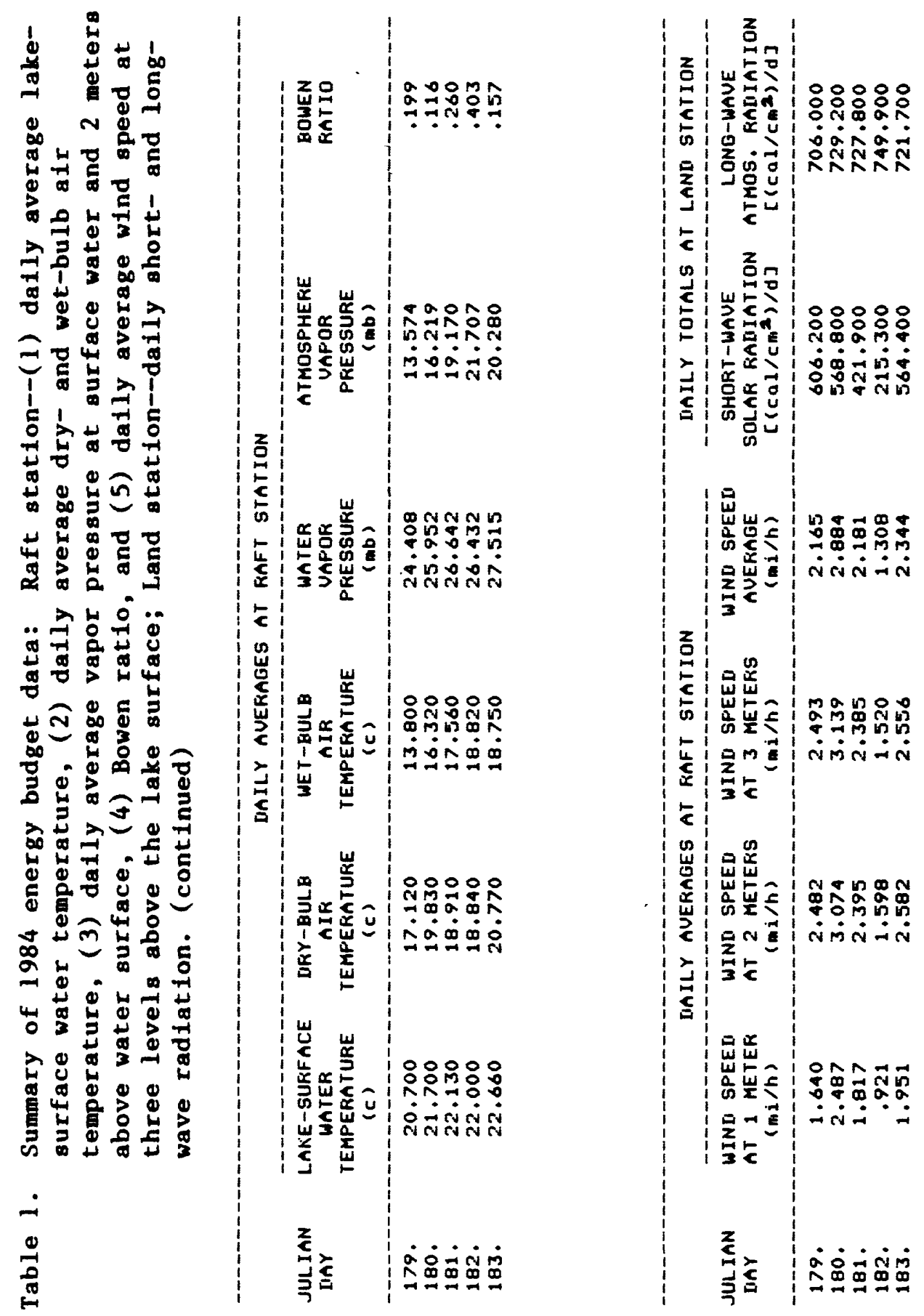



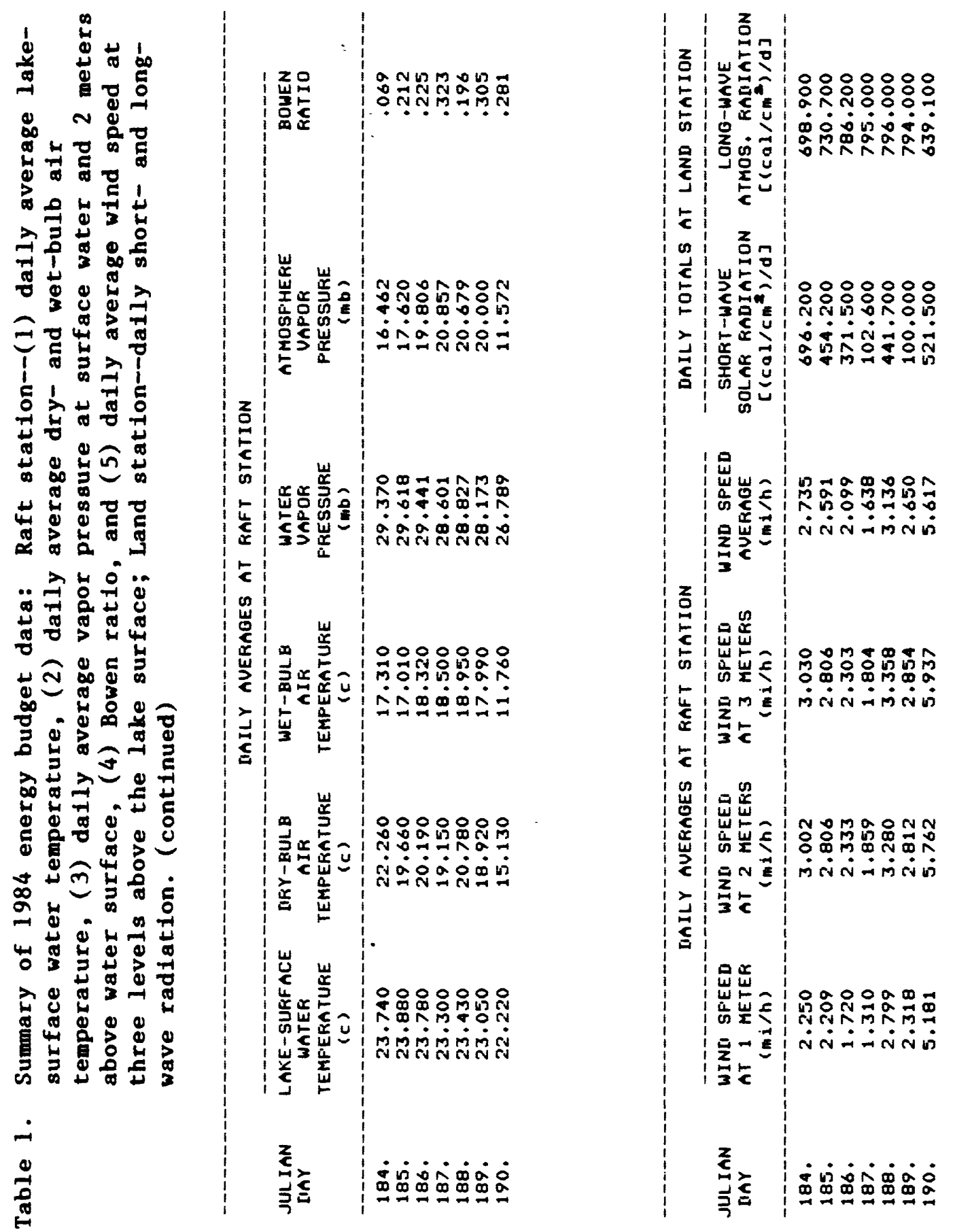

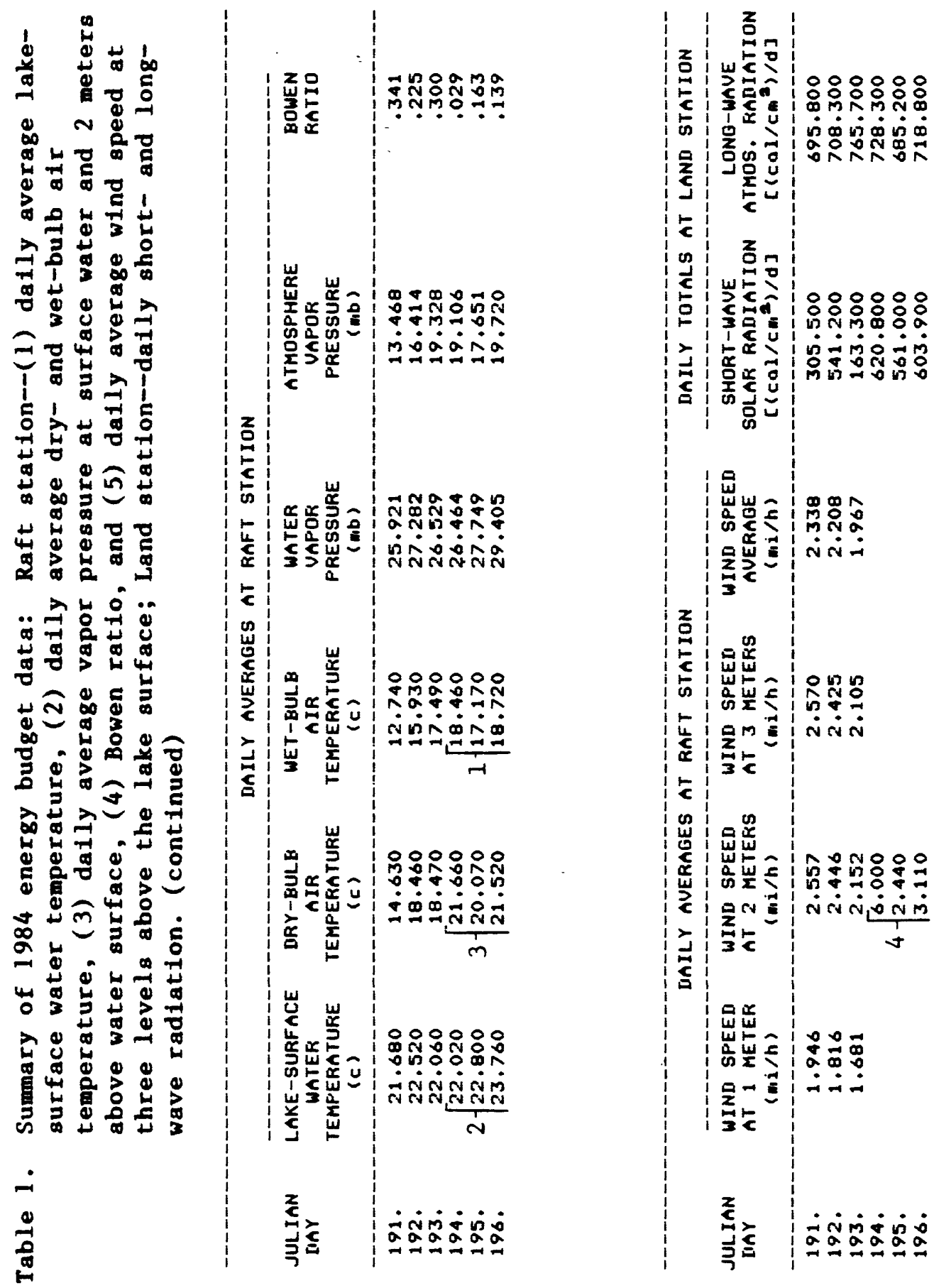

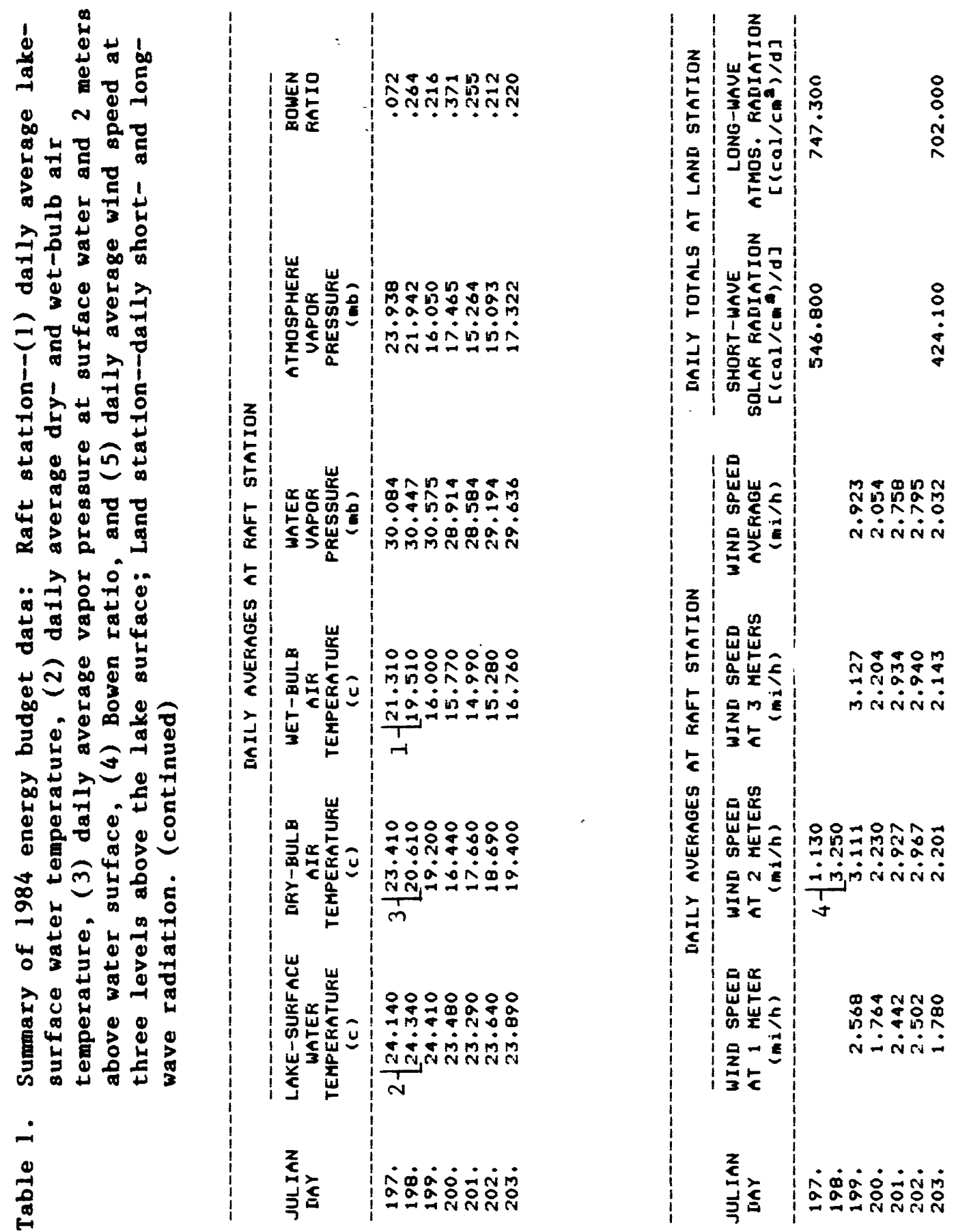

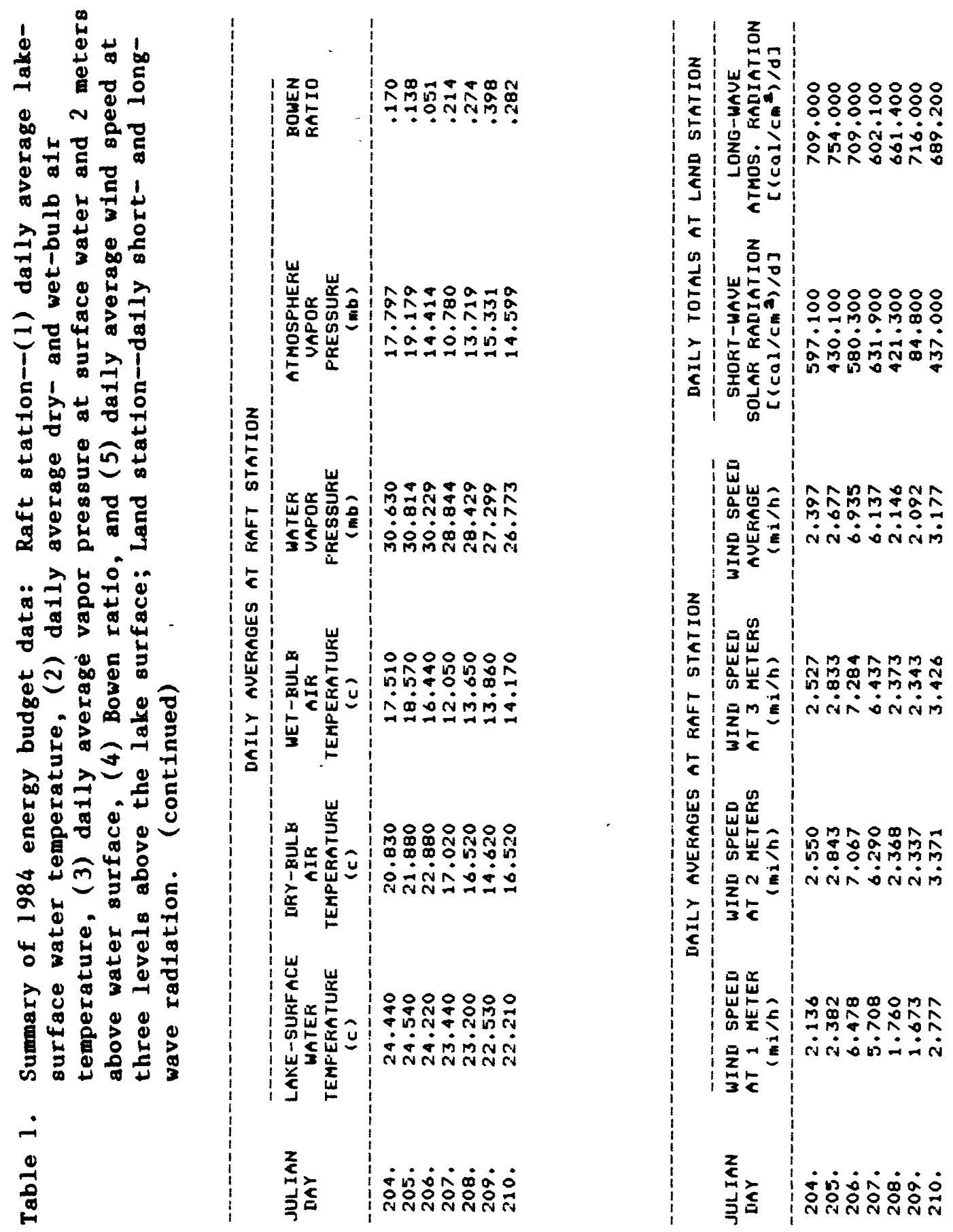

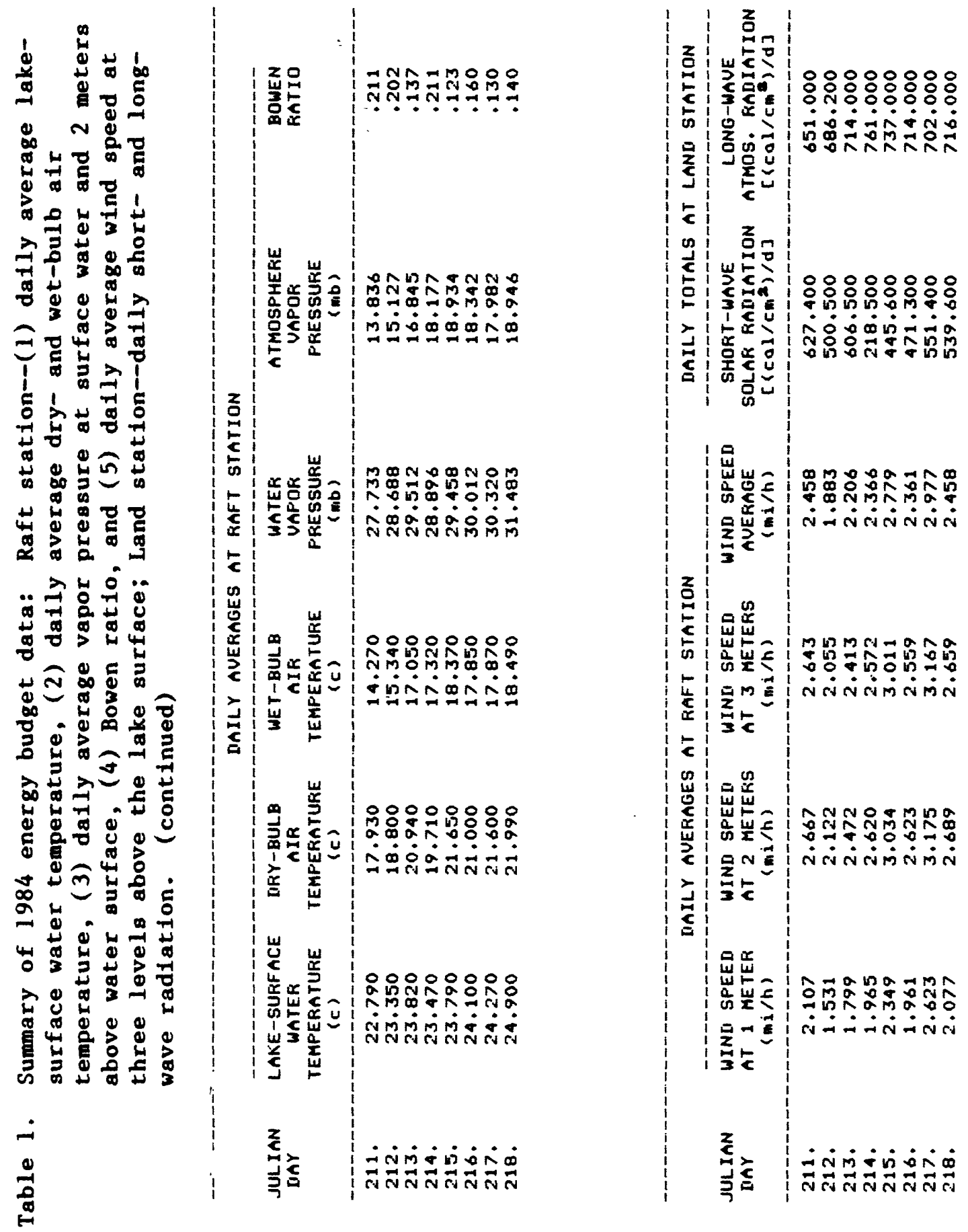

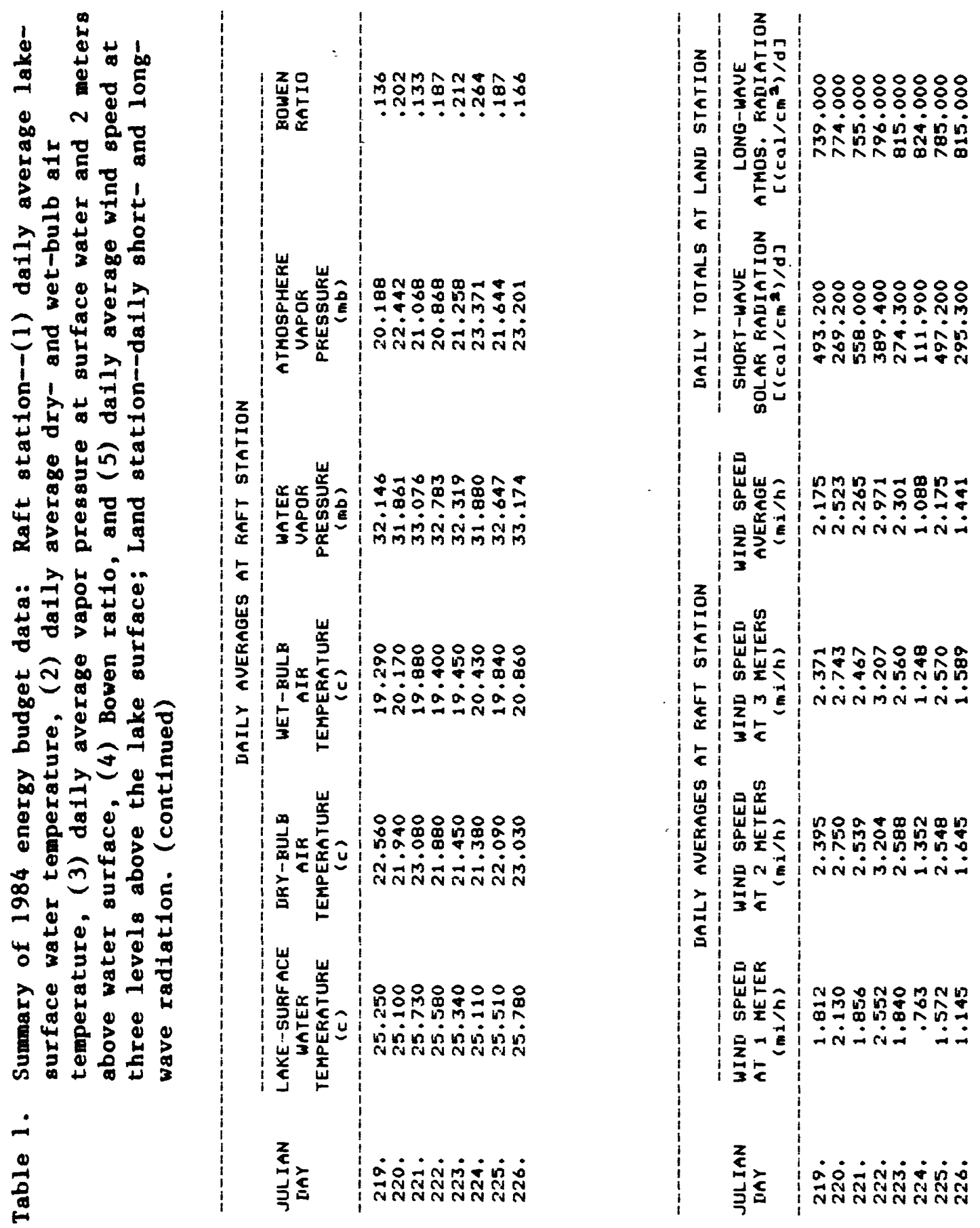

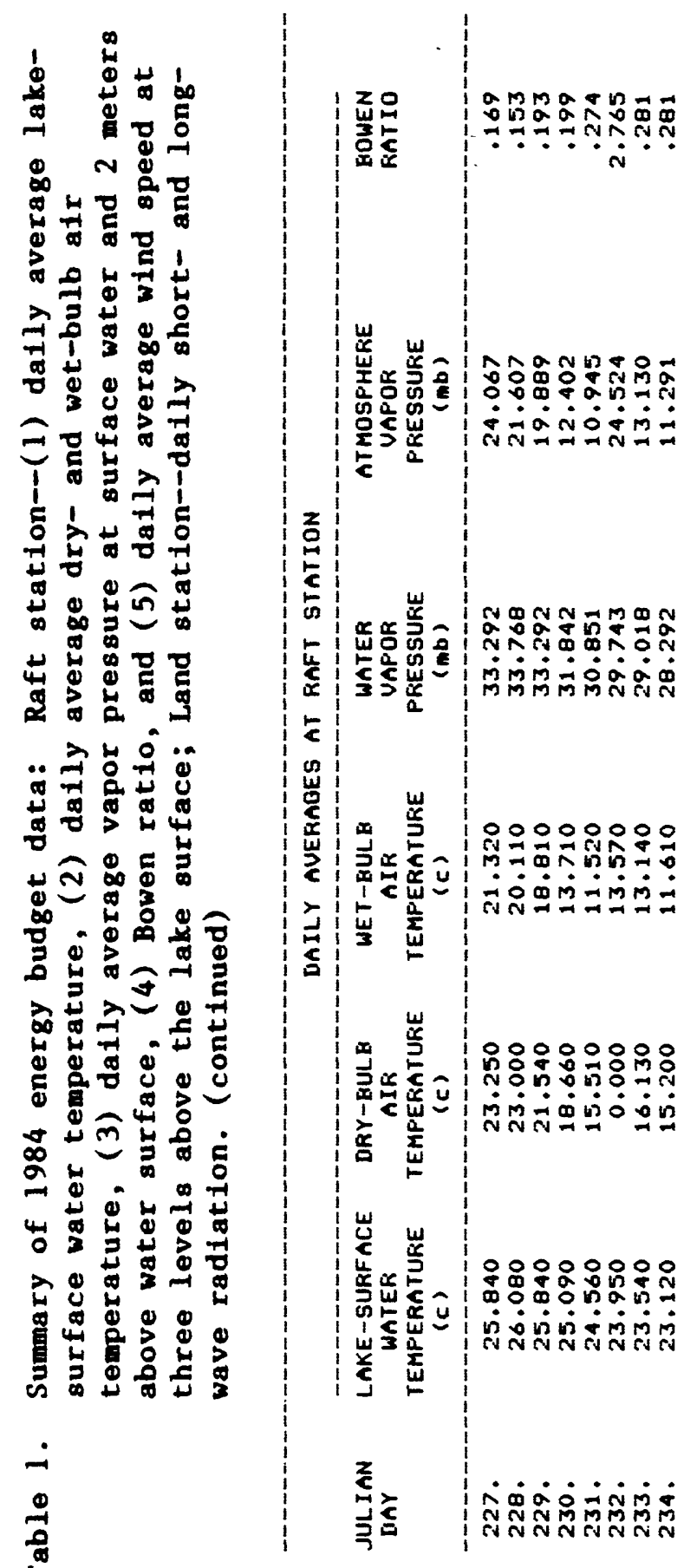

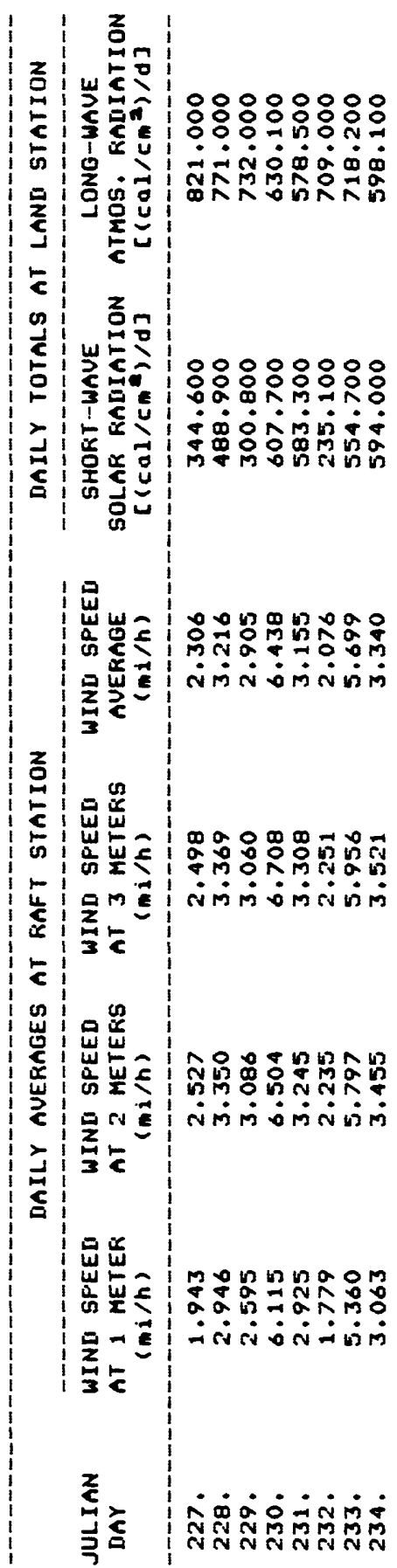



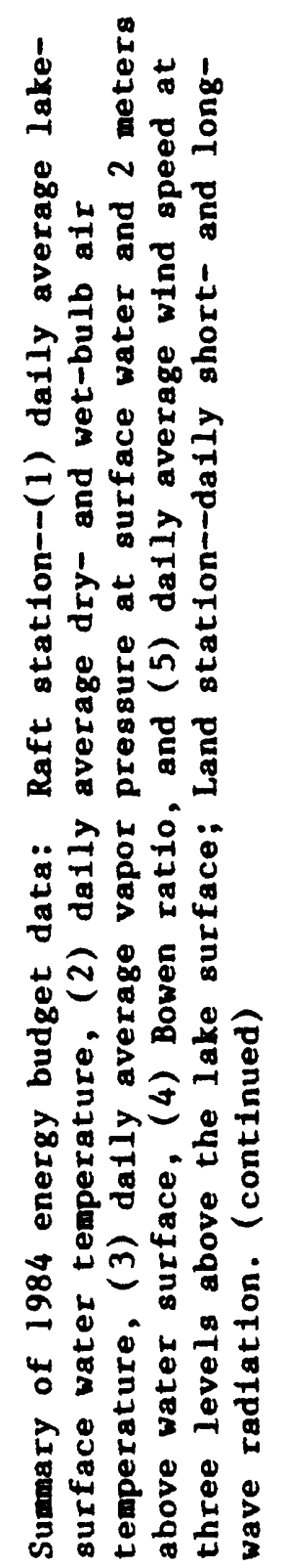

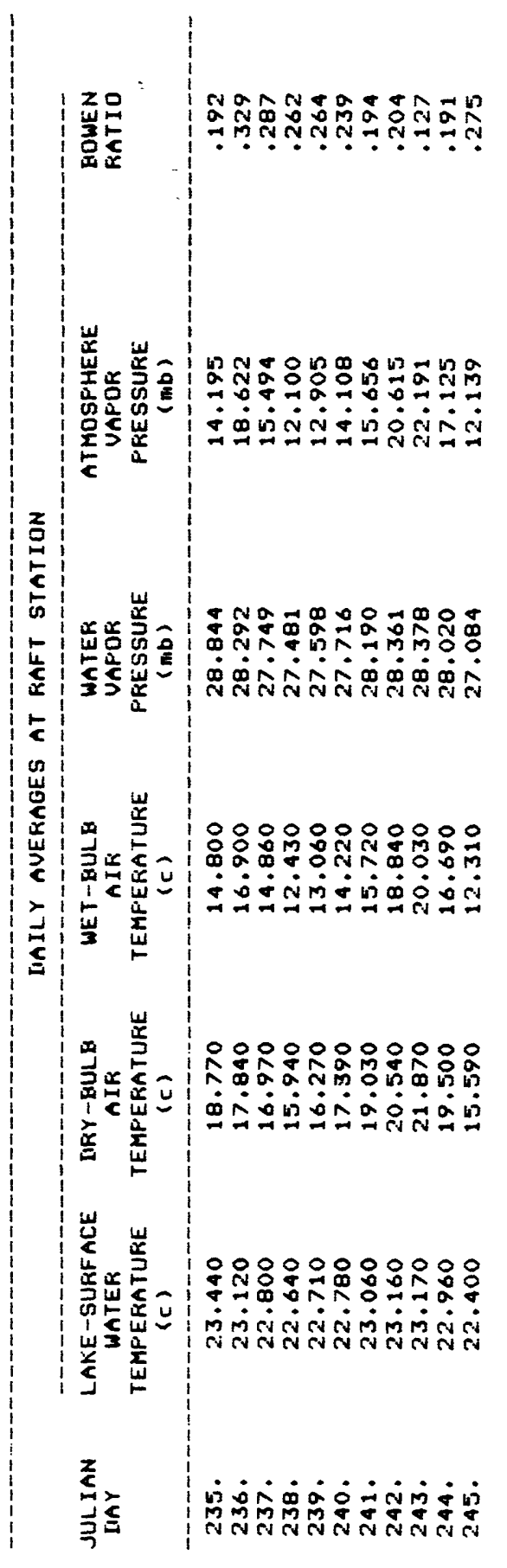

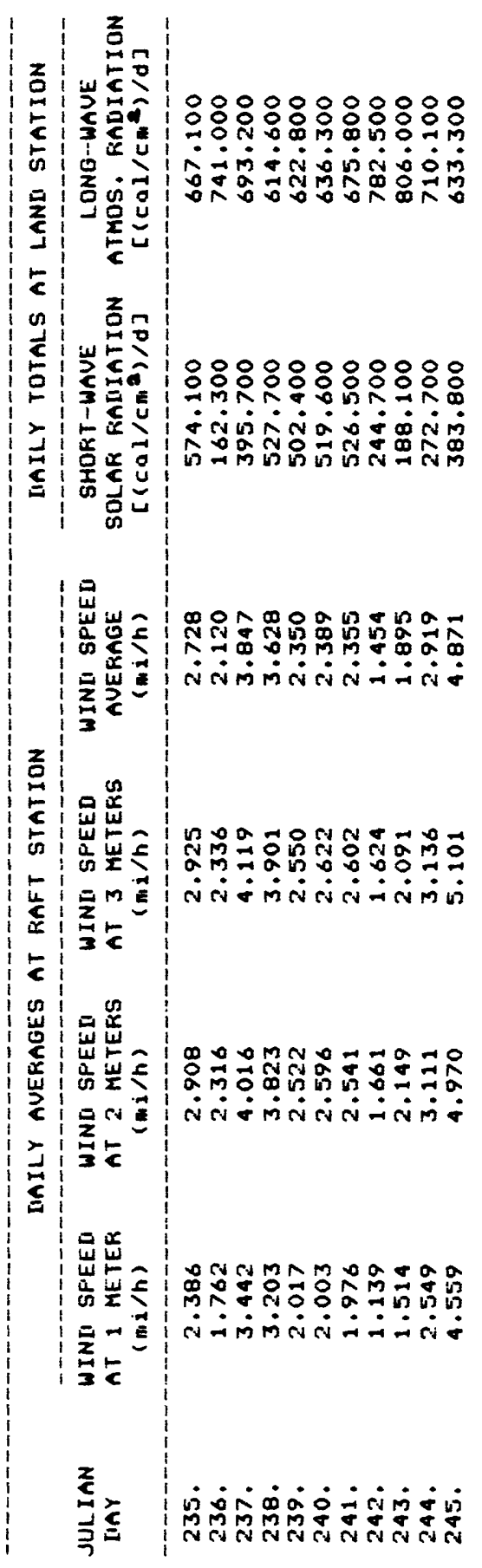



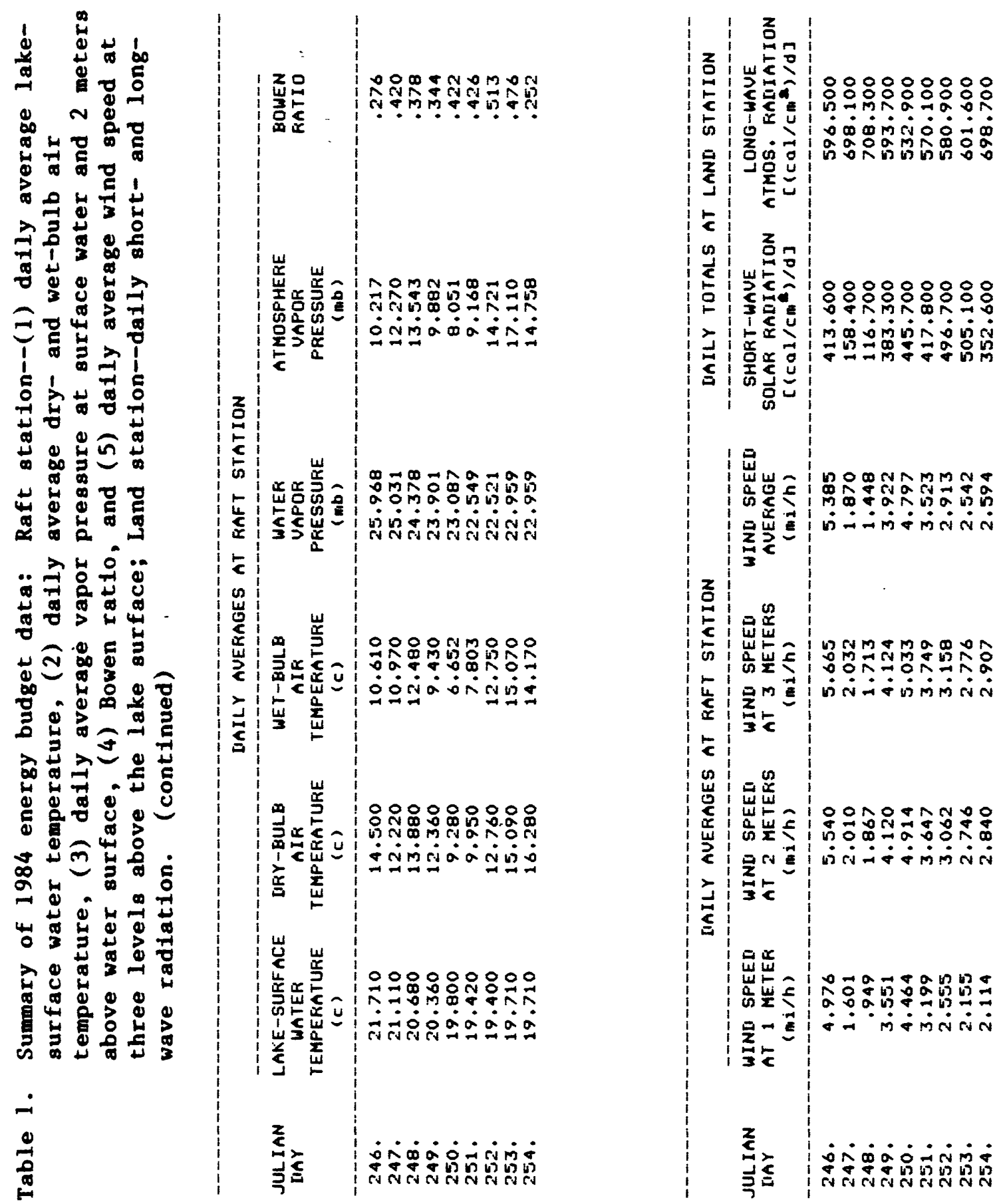

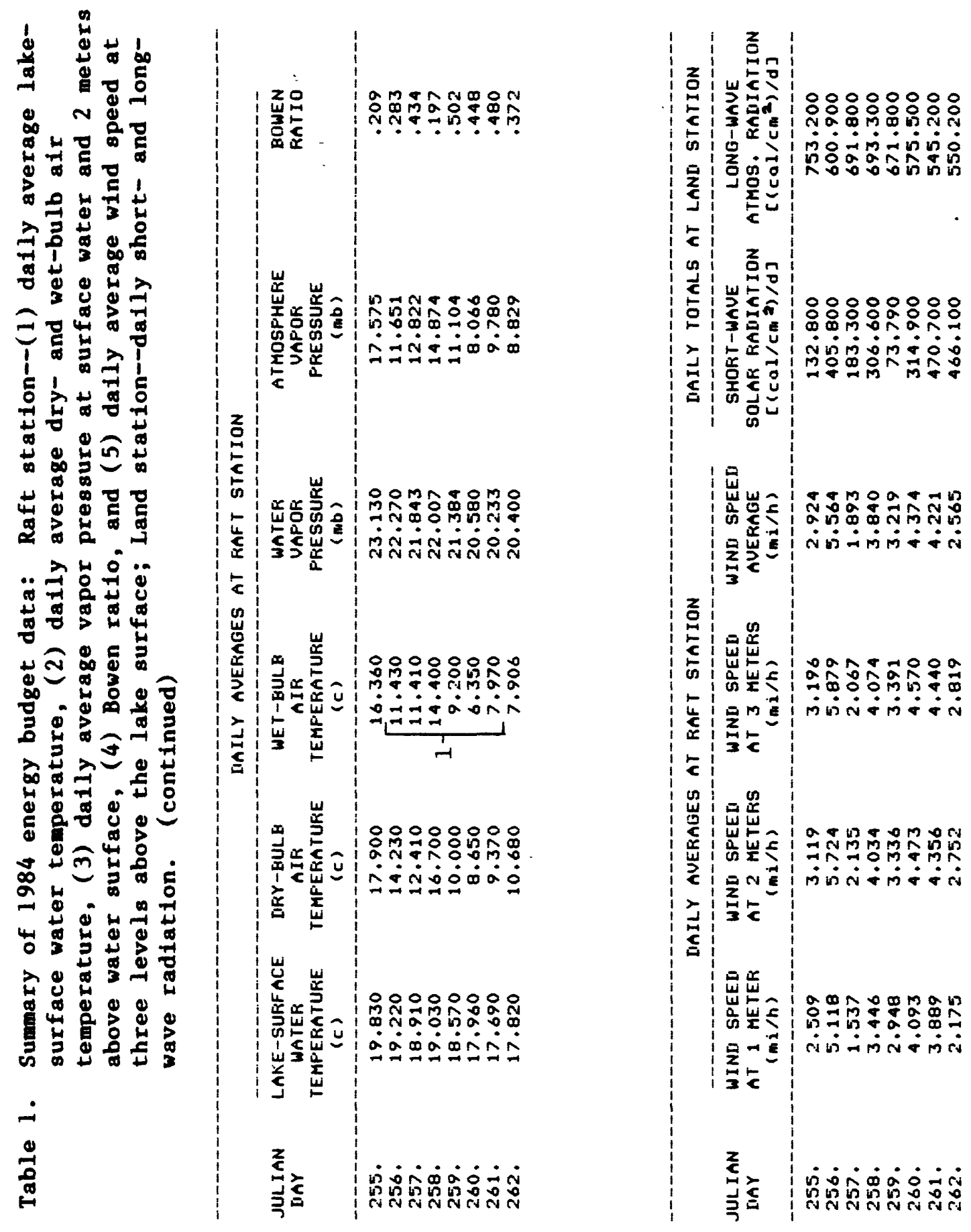

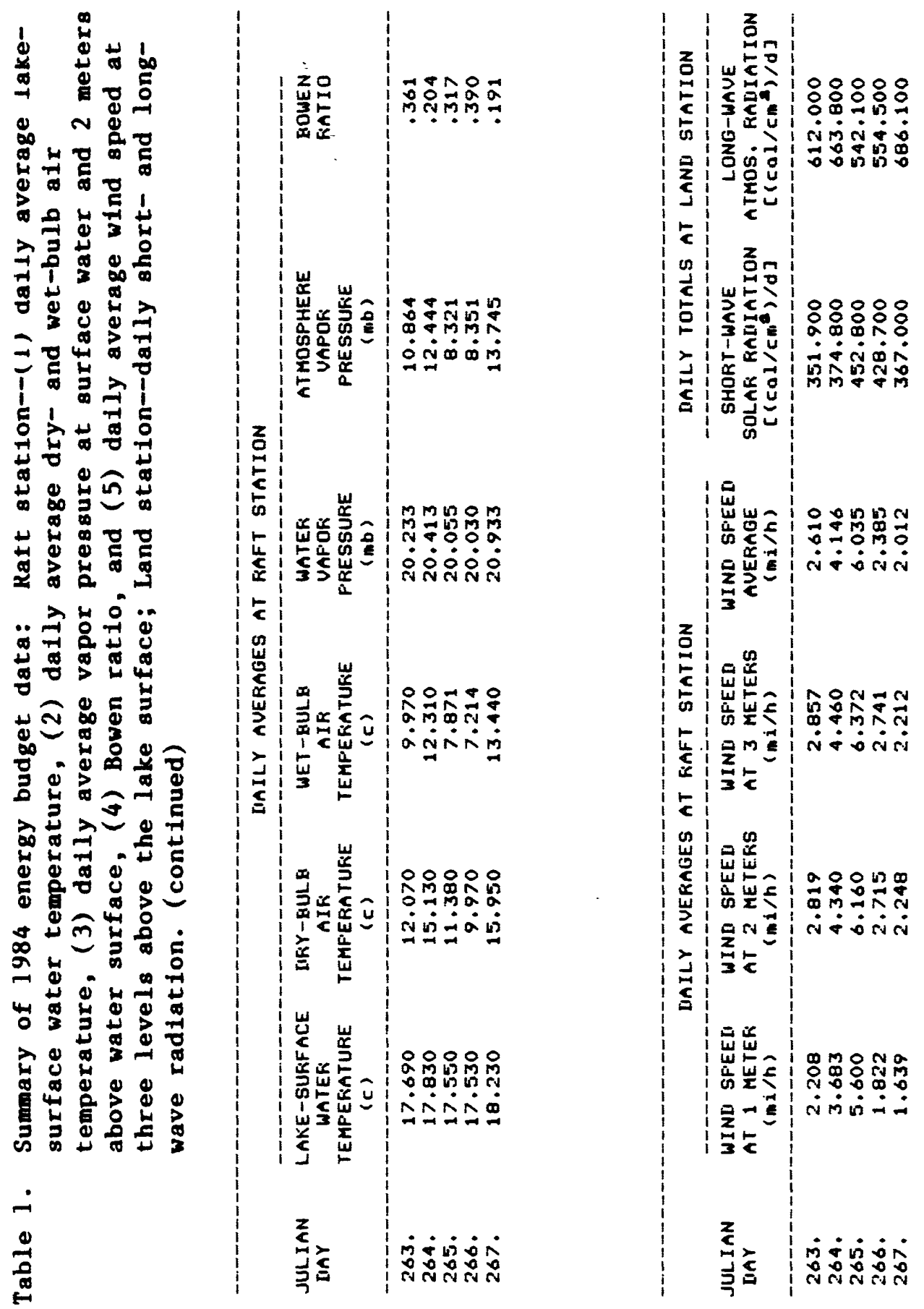



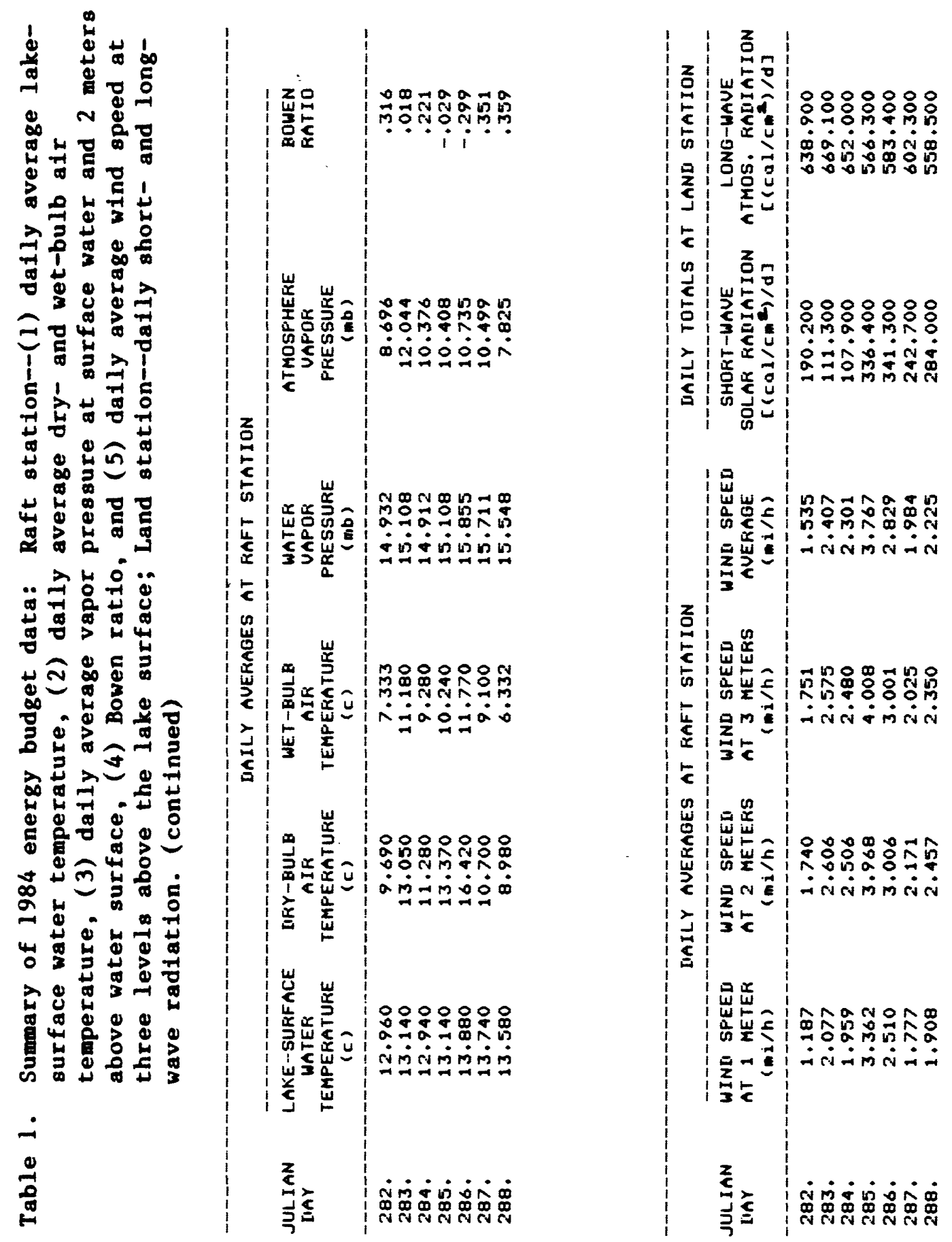

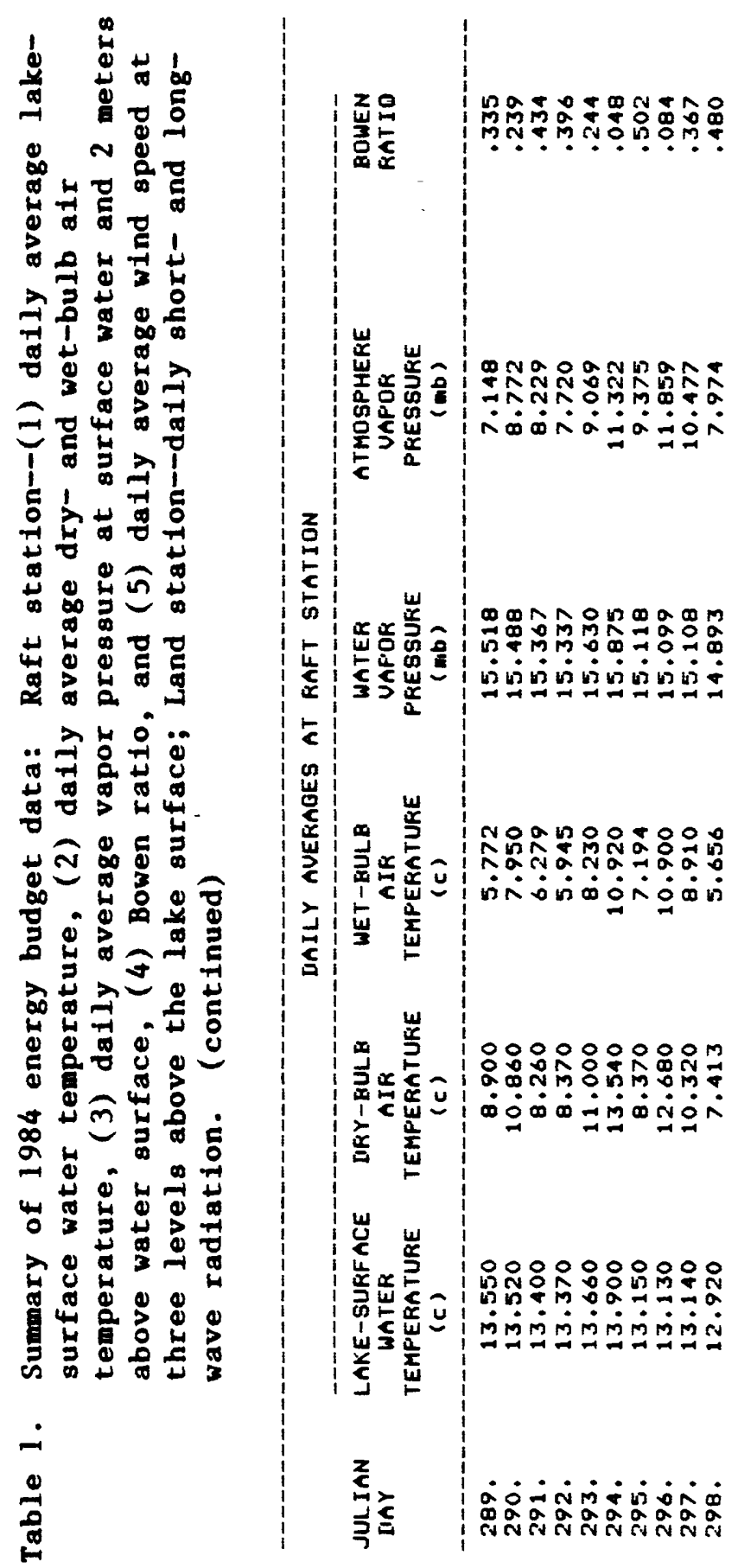

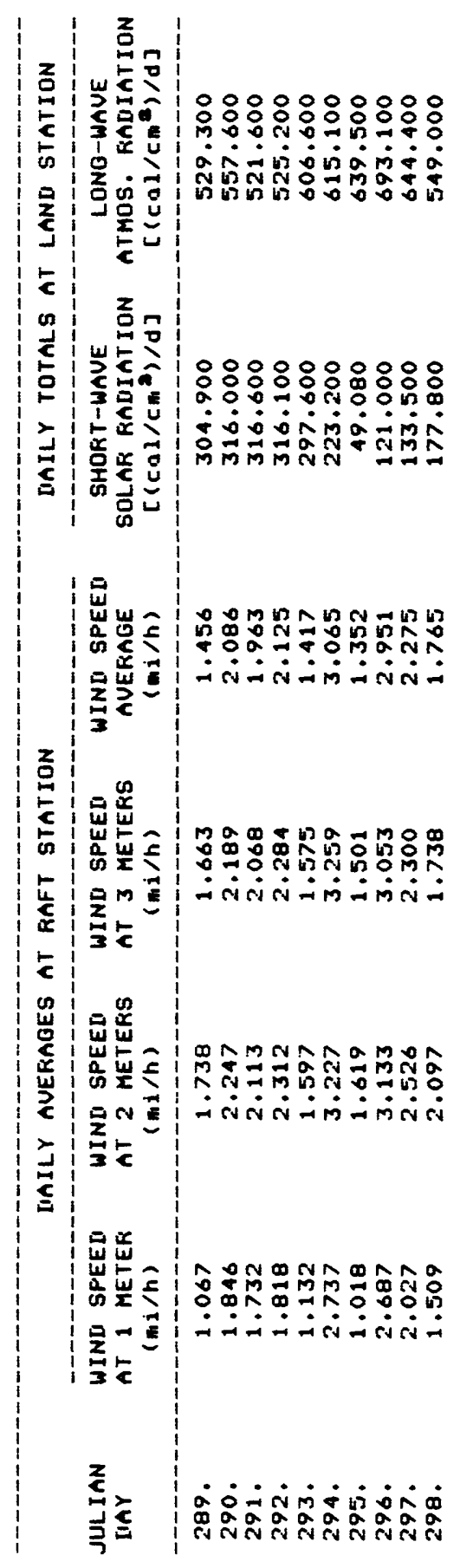



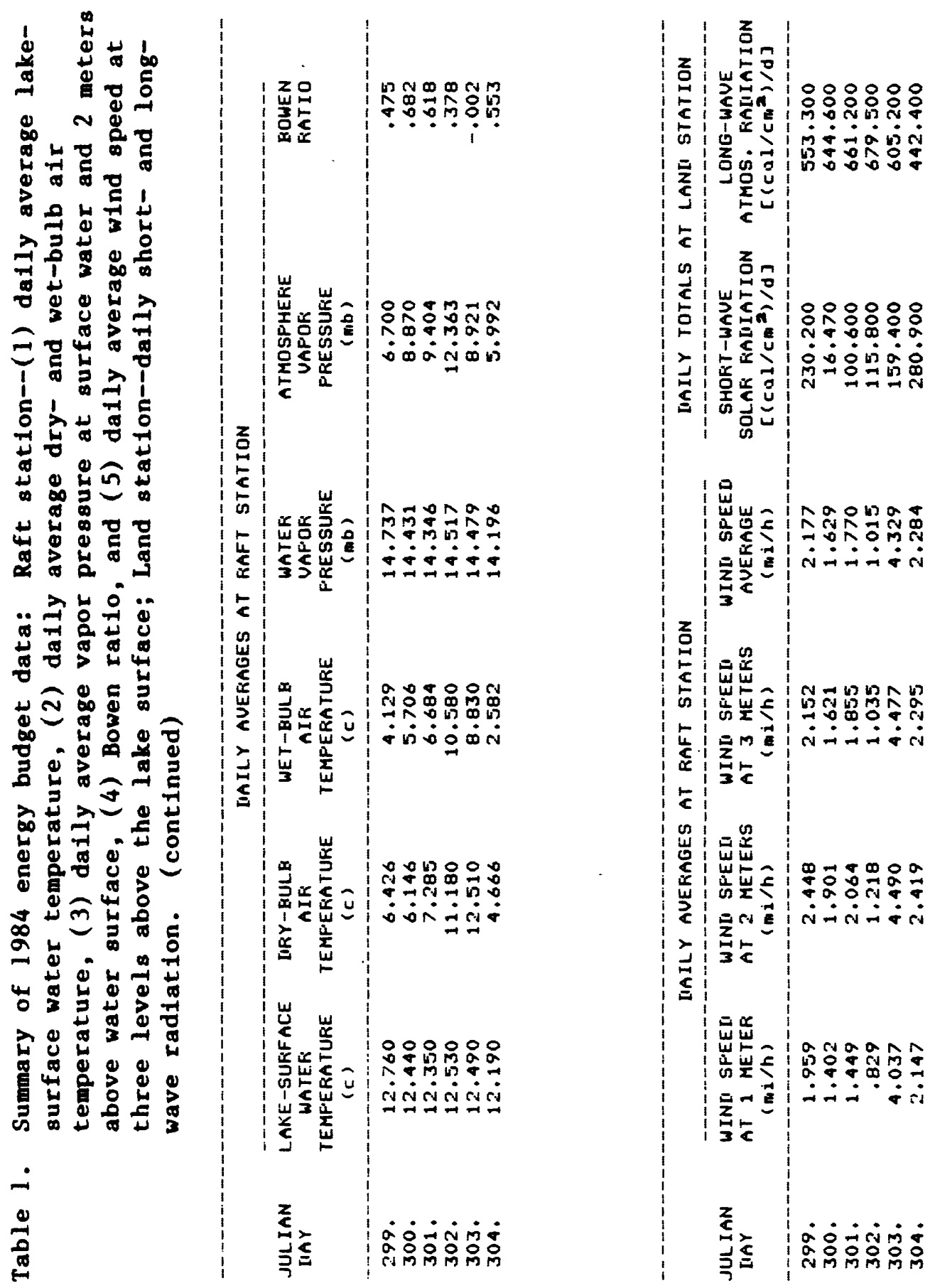


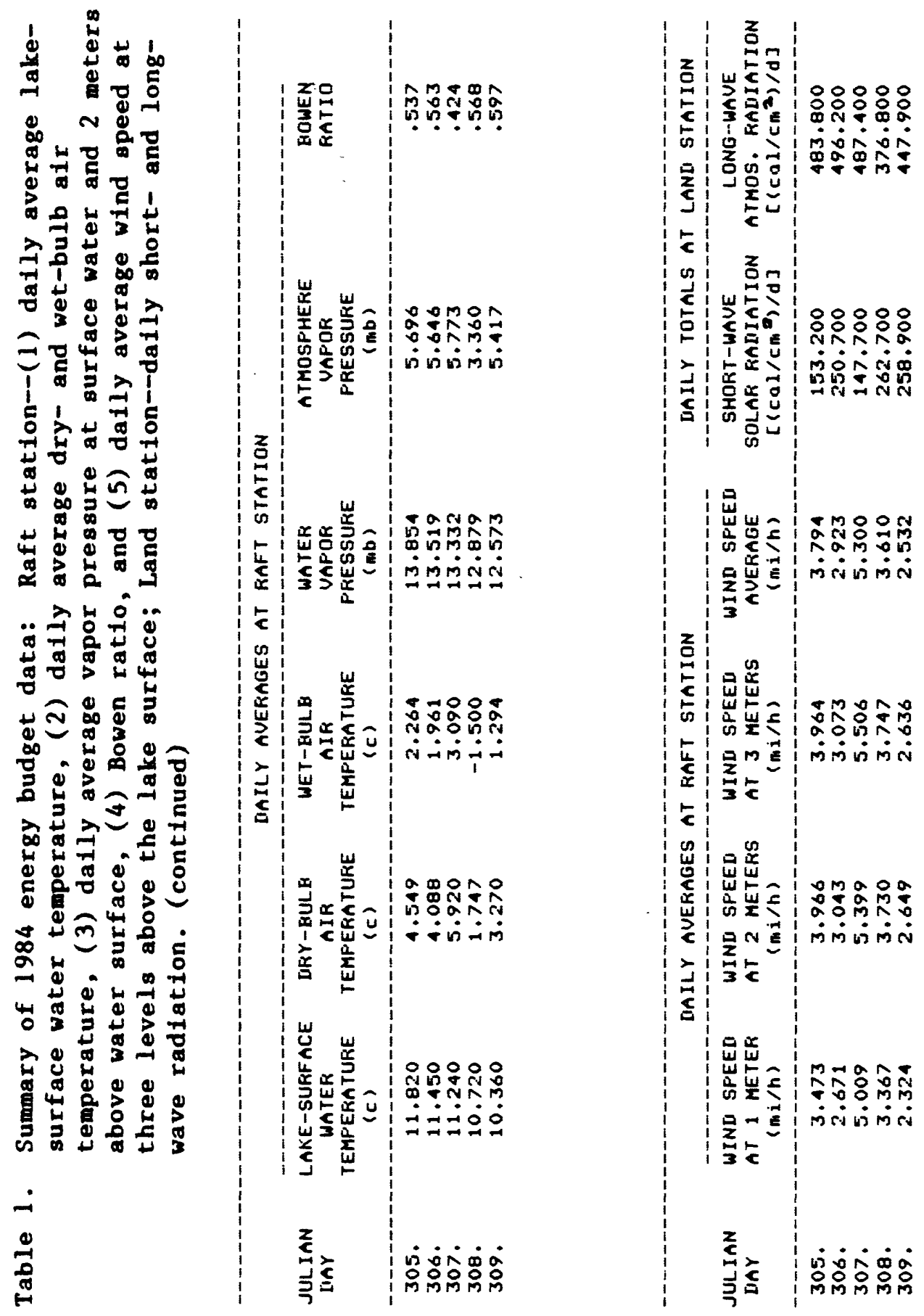



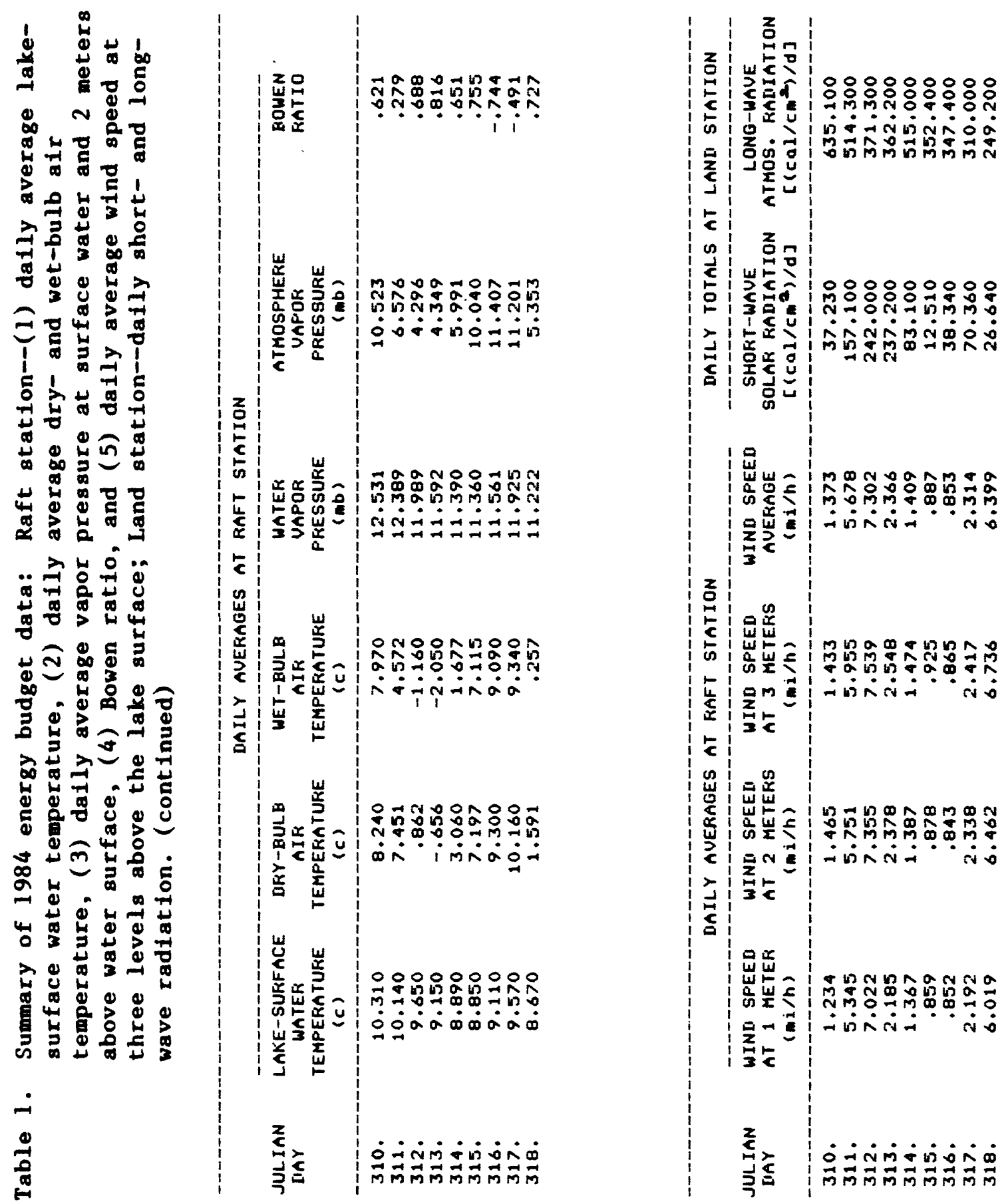

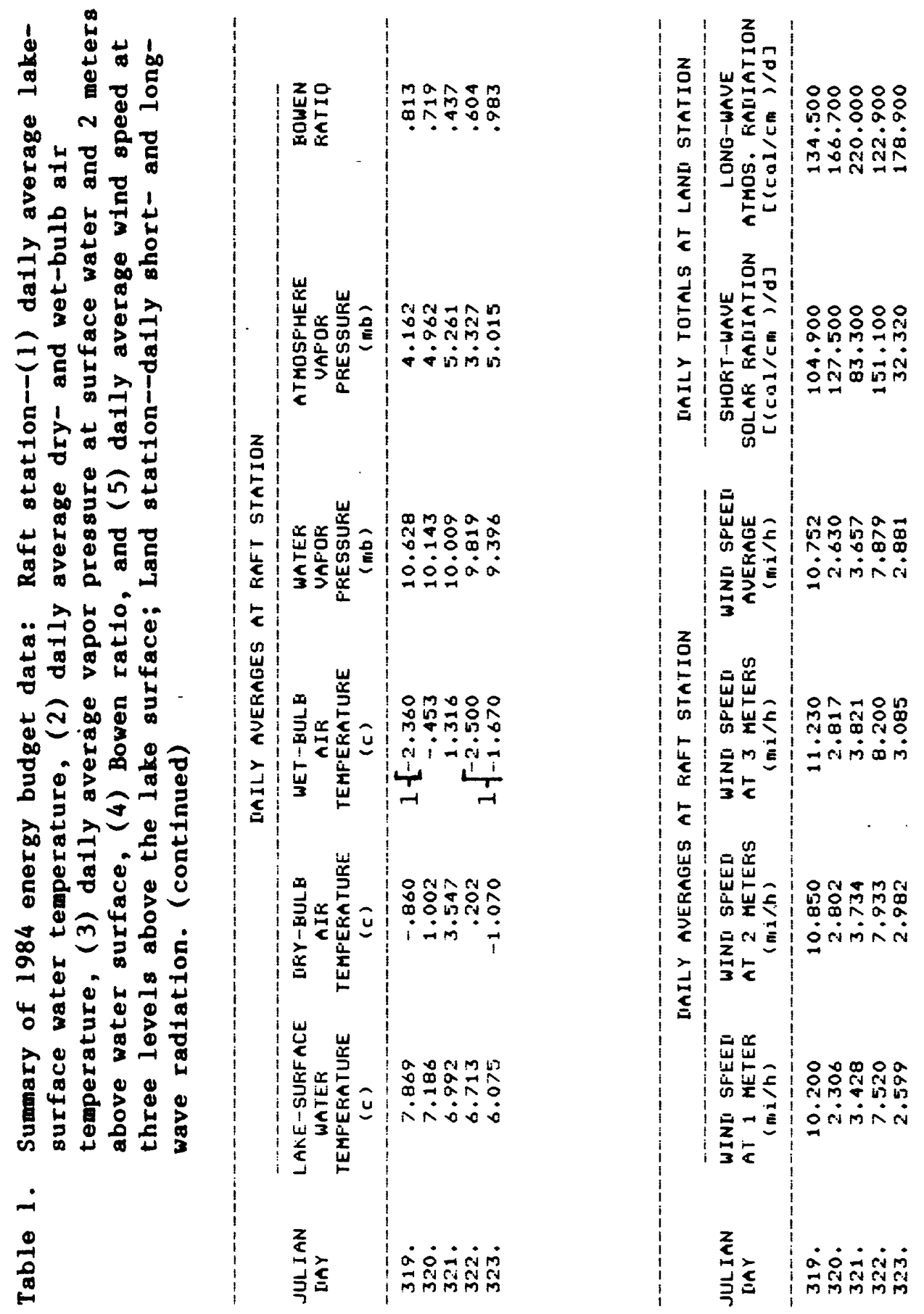

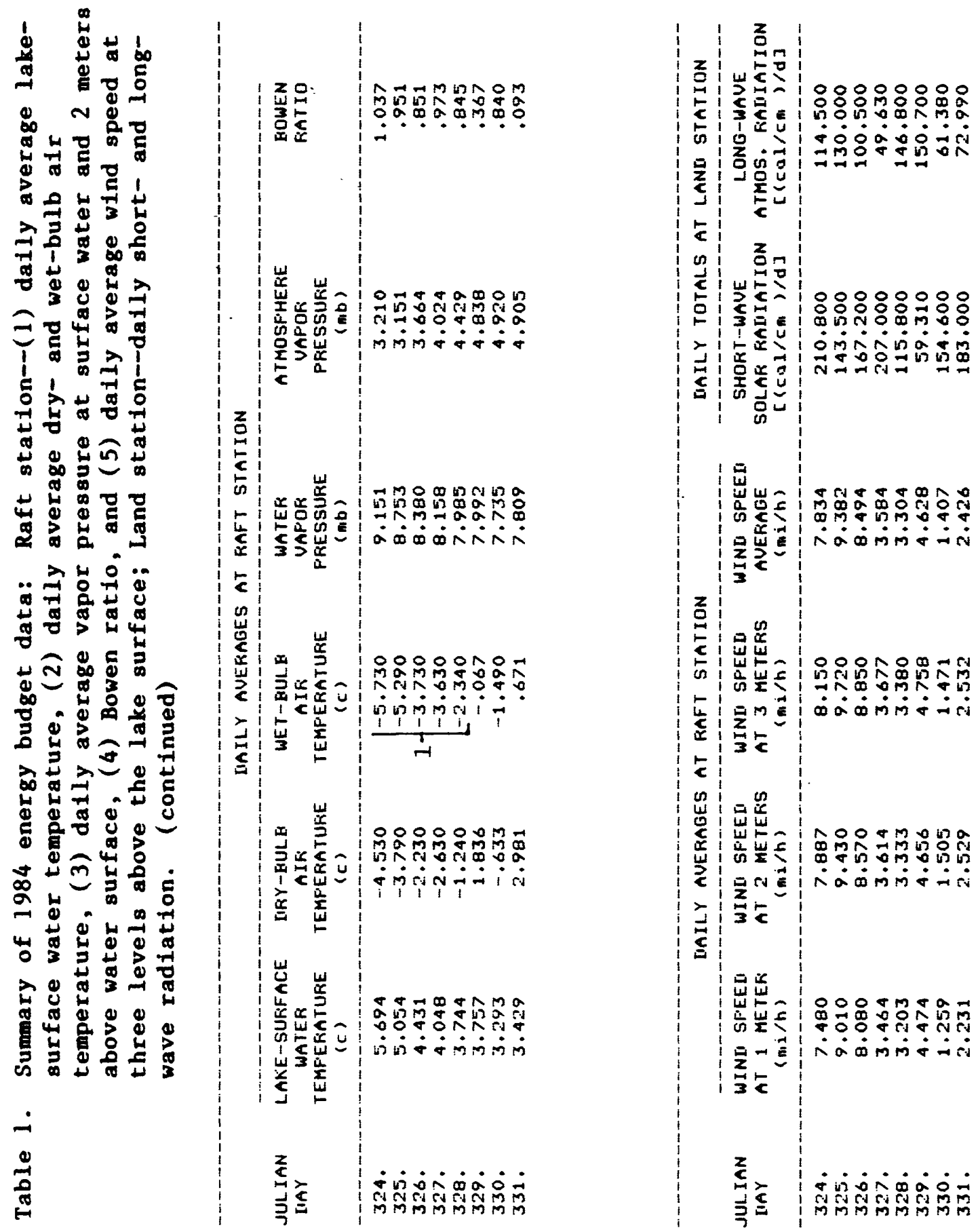

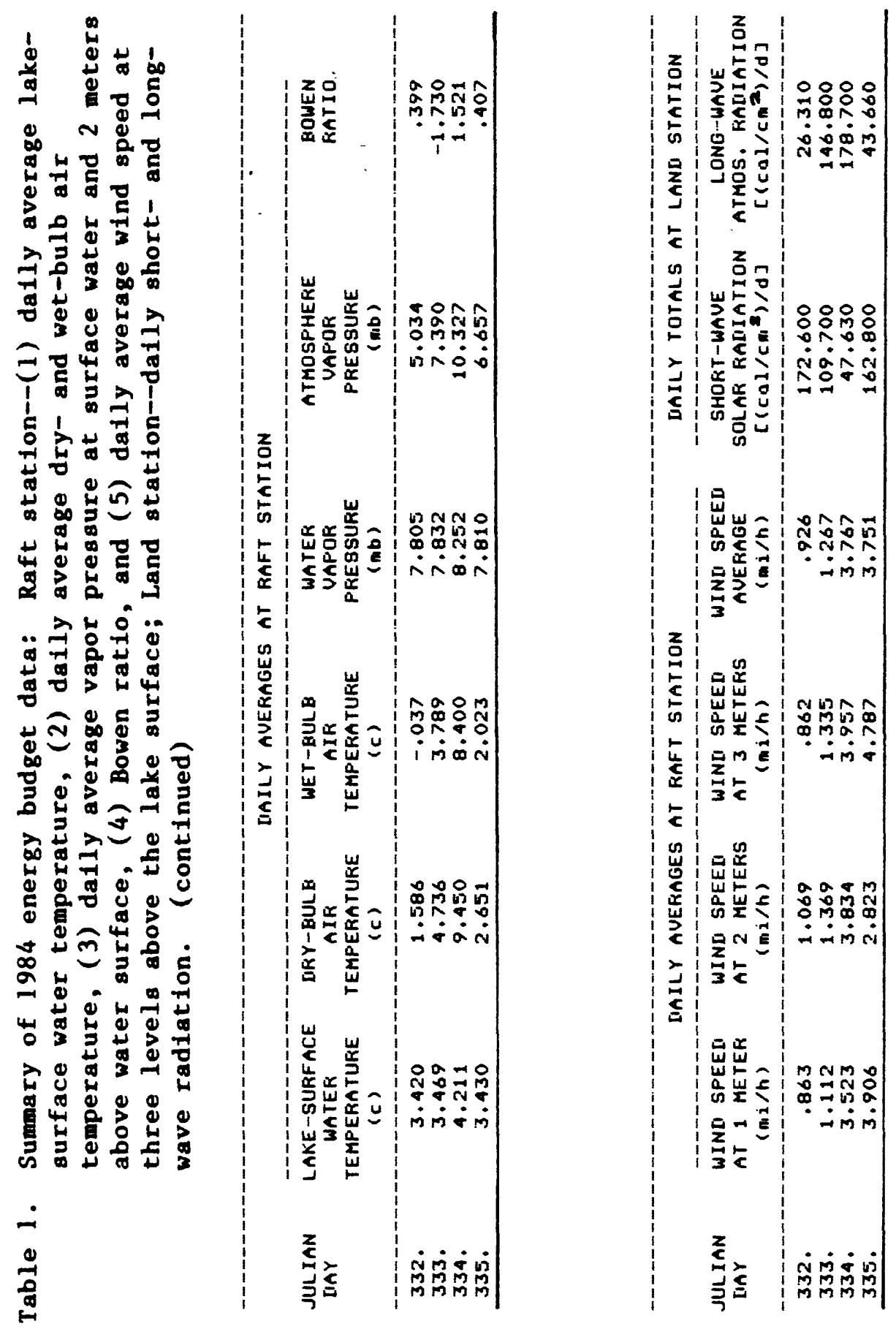

(30) 
Footnotes to table 1

${ }^{1}$ Estimated wet-bulb temperatures using psychrometric tables and air temperature and relative humidity data taken from hygrothermograph.

${ }^{2}$ Calculated by regression equation 1 (see below), which was determined by using data from the Marshalltown water temperature recorded and the thermistor connected to the CR-21.

${ }^{3}$ Calculated by regression equation 2 (see below), which was determined by using air temperature data from the hygrothermograph and the dry-bulb sensor connected to the CR-21.

${ }^{4}$ Estimated using data from Belfort totalizing anemometer, also positioned at 2 meters above the water surface on the raft.

\begin{tabular}{lcl}
\hline Regression & Data points & $\mathrm{r}^{2}$ value \\
\hline $1 . \quad Y=1.937+0.964 \times$ & 20 & 0.89 \\
2. $Y=1.002+1.076 \times$ & 20 & 0.99 \\
\hline
\end{tabular}


Table 2. Summary of 1984 data: Raft station temperature--(1) da1ly average lake surface water temperature, (2) daily average dry-bulb alr temperature, (3) daily maximum and mintmum dry-bulb air temperature and the time they occurred, and (4) dally average wet-bulb afr temperature. [C, degrees Celsius, h, hour; blank, no data]

\begin{tabular}{|c|c|c|c|c|c|c|c|}
\hline $\begin{array}{l}\text { JUL I AN } \\
\text { IIAY }\end{array}$ & $\begin{array}{c}\text { IIAILY } \\
\text { AUERAGE } \\
\text { LAKE-SURFFAE } \\
\text { WATEF } \\
\text { TEMFERATURE } \\
\text { (C) }\end{array}$ & $\begin{array}{l}\text { IIAILY } \\
\text { AUERAGE } \\
\text { IIFY - BULE } \\
\text { AIR } \\
\text { TEMFERATUFE } \\
\text { (C) }\end{array}$ & $\begin{array}{c}\text { MAXIMUM } \\
\text { LIRY-EULE } \\
\text { AIR } \\
\text { TEMFERATURE } \\
\text { (C) }\end{array}$ & $\begin{array}{l}\text { TIME OF } \\
\text { MAXIMUM } \\
\text { IRY-BULE } \\
\text { AIR } \\
\text { TEMFERATURE } \\
\text { (h) }\end{array}$ & $\begin{array}{c}\text { MINIMUM } \\
\text { IRY - BULE } \\
\text { AIR } \\
\text { TEMFERATURE } \\
\text { (C) }\end{array}$ & $\begin{array}{l}\text { TIME OF } \\
\text { MINIMUM } \\
\text { IIFY - EULLE } \\
\text { AIR } \\
\text { TEMFERATURE } \\
\text { (h) }\end{array}$ & $\begin{array}{l}\text { IIAILY } \\
\text { AUERAGE } \\
\text { WET-EULE } \\
\text { AIR } \\
\text { TEMFEFATURE } \\
(C)\end{array}$ \\
\hline $\begin{array}{l}114 \\
115 \\
116 \\
117 \\
118 \\
119 \\
120 \\
121 \\
122 \\
123 \\
124 \\
125 \\
126 \\
127\end{array}$ & $\begin{array}{r}7.81 \\
8.22 \\
7.84 \\
8.11 \\
9.47 \\
11.27 \\
12.65 \\
13.32 \\
12.40 \\
11.07 \\
10.91 \\
10.98 \\
10.26 \\
10.30\end{array}$ & $\begin{array}{r}9.53 \\
7.66 \\
7.11 \\
8.78 \\
9.24 \\
11.22 \\
13.47 \\
15.88 \\
9.47 \\
5.28 \\
7.31 \\
7.57 \\
5.67 \\
9.49\end{array}$ & $\begin{array}{r}19.55 \\
10.31 \\
8.82 \\
15.86 \\
18.23 \\
20.78 \\
24.65 \\
25.62 \\
12.60 \\
7.59 \\
15.06 \\
9.79 \\
7.68 \\
18.23\end{array}$ & $\begin{array}{r}1522 \\
1509 \\
1503 \\
1642 \\
1631 \\
1505 \\
1654 \\
1318 \\
4 \\
1605 \\
1603 \\
928 \\
1830 \\
1707\end{array}$ & $\begin{array}{r}-1.29 \\
5.66 \\
5.22 \\
3.19 \\
-.68 \\
.82 \\
3.28 \\
6.10 \\
6.18 \\
-.50 \\
-2.78 \\
5.48 \\
3.02 \\
-.06\end{array}$ & $\begin{array}{r}521 \\
605 \\
2400 \\
2359 \\
422 \\
524 \\
326 \\
459 \\
505 \\
2357 \\
455 \\
2353 \\
2400 \\
520\end{array}$ & $\begin{array}{r}4.43 \\
6.07 \\
5.34 \\
4.59 \\
5.37 \\
6.17 \\
8.51 \\
11.00 \\
4.66 \\
1.77 \\
3.57 \\
6.44 \\
3.83 \\
5.51\end{array}$ \\
\hline
\end{tabular}

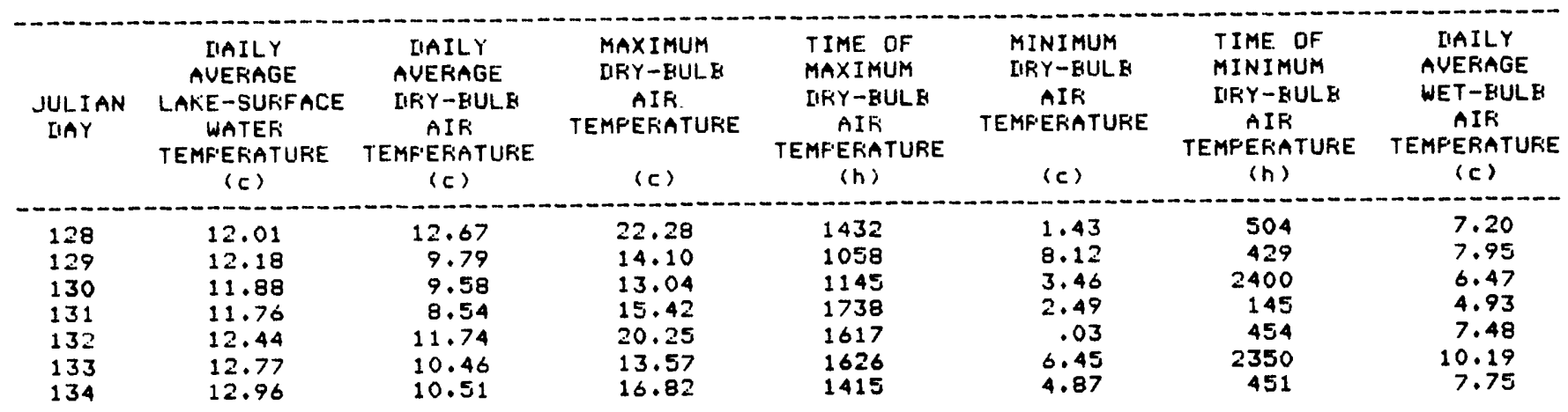

\begin{tabular}{|c|c|c|c|c|c|c|c|}
\hline $\begin{array}{l}\text { JUL I AN } \\
\text { IIAY }\end{array}$ & $\begin{array}{c}\text { IIAILY } \\
\text { AUERAGE } \\
\text { LAKE-SURFACE } \\
\text { WATER } \\
\text { TEMFERATURE } \\
\text { (C) }\end{array}$ & $\begin{array}{l}\text { IIAILY } \\
\text { AUERAGE } \\
\text { IIRY - EULE } \\
\text { AIR } \\
\text { TEMFEFIATURE } \\
\text { (C) }\end{array}$ & $\begin{array}{c}\text { MAXIMUM } \\
\text { IIFY-EULE } \\
\text { AIR } \\
\text { TEMFERATURE } \\
\text { (C) }\end{array}$ & $\begin{array}{l}\text { TIME OF } \\
\text { MAXIMUM } \\
\text { IIRY - BULE } \\
\text { AIR } \\
\text { TEMFERATURE } \\
\text { (h) }\end{array}$ & $\begin{array}{l}\text { MINIMUM } \\
\text { LRY - EULLE } \\
\text { AIR } \\
\text { TEMFERATURE } \\
\text { (C) }\end{array}$ & $\begin{array}{c}\text { TIME OF } \\
\text { MINIMUM } \\
\text { IIRY-EULE } \\
\text { AIR } \\
\text { TEMFERATURE } \\
\text { (h) }\end{array}$ & $\begin{array}{l}\text { IIAILY } \\
\text { AUERAGE } \\
\text { WET-EULE } \\
\text { AIR } \\
\text { TEMPERATURE } \\
\text { (C) }\end{array}$ \\
\hline $\begin{array}{l}135 \\
136 \\
137 \\
138 \\
139 \\
140 \\
141\end{array}$ & $\begin{array}{l}12.62 \\
12.09 \\
11.80 \\
11.80 \\
12.32 \\
12.63 \\
13.77\end{array}$ & $\begin{array}{r}7.24 \\
6.45 \\
5.76 \\
7.50 \\
9.51 \\
9.58 \\
15.40\end{array}$ & $\begin{array}{r}8.73 \\
10.93 \\
8.29 \\
14.27 \\
17.88 \\
18.14 \\
21.66\end{array}$ & $\begin{array}{l}1803 \\
1349 \\
1729 \\
1805 \\
1623 \\
1304 \\
1327\end{array}$ & $\begin{array}{r}5.22 \\
.64 \\
2.14 \\
-.76 \\
-.68 \\
.56 \\
8.64\end{array}$ & $\begin{array}{r}2358 \\
444 \\
2356 \\
229 \\
424 \\
401 \\
401\end{array}$ & $\begin{array}{r}5.47 \\
3.99 \\
2.99 \\
3.63 \\
4.19 \\
6.72 \\
11.83\end{array}$ \\
\hline
\end{tabular}


Table 2. Summary of 1984 data: Raft station temperature--(1) dally average lake surface water temperature, (2) daily average dry-bulb air temperature, (3) daily maximum and minimum dry-bulb air temperature and the time they occurred, and (4) daily average wet-bulb air temperature. (continued)

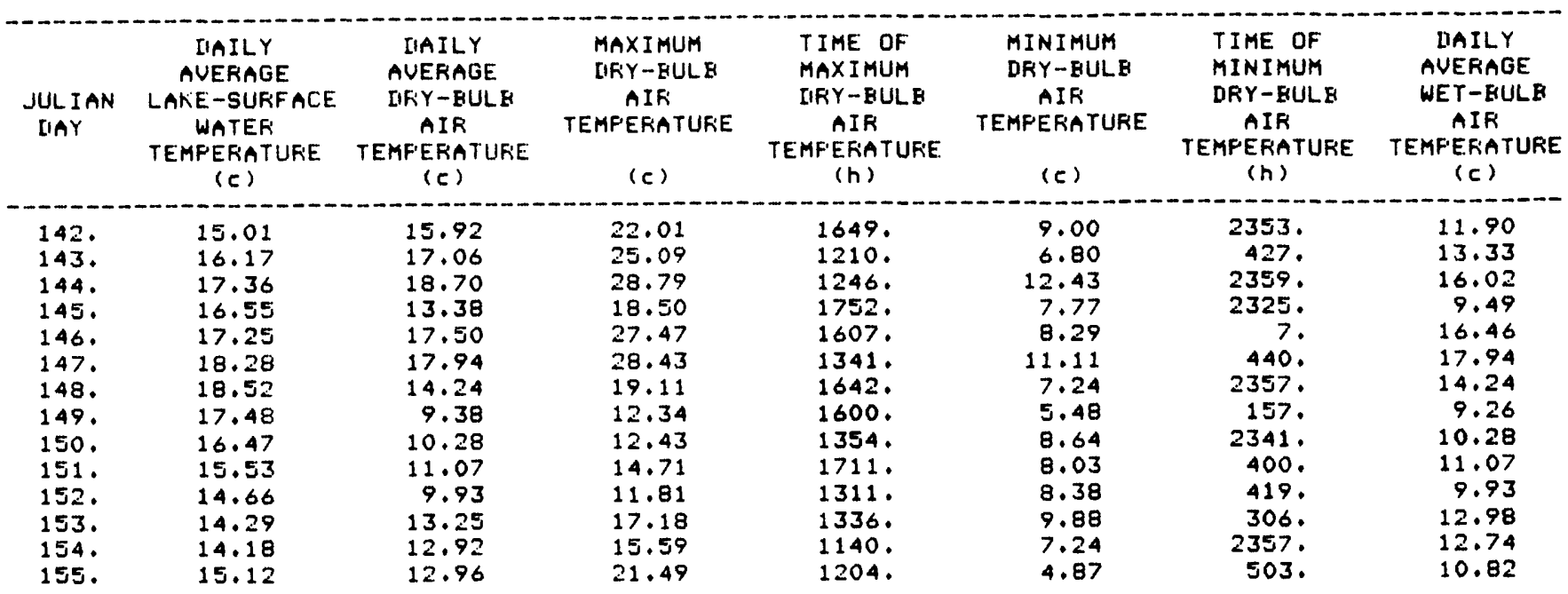

\begin{tabular}{|c|c|c|c|c|c|c|c|}
\hline $\begin{array}{l}156 . \\
157 . \\
158 . \\
159 . \\
160 .\end{array}$ & $\begin{array}{l}15.75 \\
17.18 \\
18.14 \\
20.48 \\
21.76\end{array}$ & $\begin{array}{l}15.38 \\
18.00 \\
16.07 \\
20.59 \\
23.29\end{array}$ & $\begin{array}{l}23.25 \\
26.76 \\
21.31 \\
29.58 \\
31.95\end{array}$ & $\begin{array}{l}16011^{\circ} \\
1433^{\circ} \\
10010^{\circ} \\
14580^{\circ} \\
1426 .\end{array}$ & $\begin{array}{r}7.15 \\
7.86 \\
10.75 \\
15.59 \\
15.94\end{array}$ & $\begin{array}{l}345 . \\
417 \\
441 . \\
103 \\
506 .\end{array}$ & $\begin{array}{l}11.13 \\
12.59 \\
14.17 \\
19.33 \\
18.91\end{array}$ \\
\hline
\end{tabular}

\begin{tabular}{|c|c|c|c|c|c|c|c|}
\hline $\begin{array}{l}\text { JUL IAN } \\
\text { LIAY }\end{array}$ & $\begin{array}{c}\text { IIAILY } \\
\text { AUEFAGE } \\
\text { LAKE-SUFFFACE } \\
\text { WATEF' } \\
\text { TEMFEFIATUFE } \\
\text { (C) }\end{array}$ & $\begin{array}{l}\text { DIAILY } \\
\text { AUERAGE } \\
\text { DRY-BULE } \\
\text { AIR } \\
\text { TEMFERATURE } \\
\text { (C) }\end{array}$ & $\begin{array}{c}\text { MAXIMUM } \\
\text { IIKY-BULE } \\
\text { AIF } \\
\text { TEMF'EFATURE } \\
\text { (C) }\end{array}$ & $\begin{array}{c}\text { TIME OF } \\
\text { MAXIMUM } \\
\text { IIRY - EULE } \\
\text { AIF } \\
\text { TEMFERATURE } \\
\text { ( } h \text { ) }\end{array}$ & $\begin{array}{c}\text { MINIMUM } \\
\text { IURY - BULE } \\
\text { AIF } \\
\text { TEMFEFATURE } \\
\text { (C) }\end{array}$ & $\begin{array}{c}\text { TIME OF } \\
\text { MINIMUM } \\
\text { IIRY-EULE } \\
\text { AIR } \\
\text { TEMFERATURE } \\
\text { (h) }\end{array}$ & $\begin{array}{c}\text { IIAILYY } \\
\text { AUERAGE } \\
\text { WET - EULLE } \\
\text { AIR } \\
\text { TEMFERATURE } \\
\text { (C) }\end{array}$ \\
\hline $\begin{array}{l}161 . \\
162^{\circ} \\
163 . \\
164 . \\
165^{\circ} \\
166 . \\
1670^{\circ} \\
168 . \\
169 . \\
170 . \\
171 . \\
172 . \\
173 .\end{array}$ & $\begin{array}{l}23.06 \\
24.10 \\
24.21 \\
23.15 \\
23.45 \\
22.79 \\
20.17 \\
20.11 \\
20.71 \\
20.18 \\
21.22 \\
20.62 \\
20.45\end{array}$ & $\begin{array}{l}24.78 \\
23.58 \\
23.32 \\
21.16 \\
21.44 \\
17.84 \\
11.91 \\
13.74 \\
16.99 \\
15.34 \\
21.92 \\
16.72 \\
16.08\end{array}$ & $\begin{array}{l}31.25 \\
31.95 \\
30.55 \\
27.82 \\
30.46 \\
25.36 \\
16.21 \\
22.01 \\
23.77 \\
18.32 \\
28.26 \\
21.22 \\
22.81\end{array}$ & $\begin{array}{l}17200^{\circ} \\
14070^{\circ} \\
1240 . \\
1426 . \\
12280^{\circ} \\
14577^{\circ} \\
17460^{\circ} \\
1547{ }^{\circ} \\
1624{ }^{\circ} \\
23280^{\circ} \\
1315{ }^{\circ} \\
1530 . \\
1614 .\end{array}$ & $\begin{array}{r}16.65 \\
15.42 \\
16.56 \\
14.27 \\
11.90 \\
12.87 \\
5.39 \\
3.63 \\
10.58 \\
11.63 \\
17.35 \\
9.52 \\
7.15\end{array}$ & $\begin{array}{r}400 . \\
413 . \\
431 . \\
454 . \\
358 . \\
2355 . \\
2356 . \\
353 . \\
435 . \\
357 . \\
152 . \\
2400 . \\
340 .\end{array}$ & $\begin{array}{r}19.12 \\
18.97 \\
17.83 \\
14.74 \\
16.88 \\
16.14 \\
7.92 \\
9.52 \\
13.25 \\
14.96 \\
18.09 \\
11.36 \\
11.05\end{array}$ \\
\hline
\end{tabular}


Table 2. Summary of 1984 data: Raft station temperature--(1) da1ly average lake surface water temperature, (2) dally average dry-bulb alr temperature, (3) datly maximum and minimum dry-bulb atr temperature and the time they occurred, and (4) dafly average wet-bulb alr temperature. (continued)

\begin{tabular}{|c|c|c|c|c|c|c|c|}
\hline $\begin{array}{l}\text { JUL. IAN } \\
\text { IIAY }\end{array}$ & $\begin{array}{c}\text { IIAILY } \\
\text { AUERAGE } \\
\text { LAKE-SUFFACE } \\
\text { WATEF } \\
\text { TEMFERATUFE } \\
\text { (C) }\end{array}$ & $\begin{array}{l}\text { IIAILY } \\
\text { AUERAGE } \\
\text { IIRY-GULE } \\
\text { AIR } \\
\text { TEMF'ERATURE } \\
\text { (C) }\end{array}$ & $\begin{array}{l}\text { MAXIMUM } \\
\text { IIRY - EULE } \\
\text { AIR } \\
\text { TEMFERATURE } \\
\text { (C) }\end{array}$ & $\begin{array}{l}\text { TIME OF } \\
\text { MAXIMUM } \\
\text { IIRY - EULE } \\
\text { AIR } \\
\text { TEMFERATURE } \\
\text { (h) }\end{array}$ & $\begin{array}{l}\text { MINIMUM } \\
\text { IRY - BULE } \\
\text { AIR } \\
\text { TEMF'ERATURE } \\
\text { (C) }\end{array}$ & $\begin{array}{l}\text { TIME OF } \\
\text { MINIMUM } \\
\text { IIRY - EULE } \\
\text { AIF } \\
\text { TEMPERATURE } \\
\text { (h) }\end{array}$ & $\begin{array}{l}\text { IIAILY } \\
\text { AUERAGE } \\
\text { WET - EULE } \\
\text { AIR } \\
\text { TEMF'ERATURE } \\
\text { (C) }\end{array}$ \\
\hline & --------- & --------- & ----- & ----------- & ----- & ---------- & ------------ \\
\hline $\begin{array}{l}174 . \\
175 . \\
176 . \\
177 . \\
178 .\end{array}$ & $\begin{array}{l}20.59 \\
20.99 \\
20.08 \\
19.93 \\
19.85\end{array}$ & $\begin{array}{l}15.07 \\
15.96 \\
12.08 \\
16.54 \\
15.20\end{array}$ & $\begin{array}{l}21.93 \\
25.53 \\
14.19 \\
23.16 \\
19.02\end{array}$ & $\begin{array}{c}1540 \\
1549 \\
1023 \\
1447 \\
921 .\end{array}$ & $\begin{array}{r}7.68 \\
5.39 \\
9.52 \\
12.69 \\
10.58\end{array}$ & $\begin{array}{r}430 \\
348 \\
145 . \\
2400 \\
315 .\end{array}$ & $\begin{array}{l}15.32 \\
13.09\end{array}$ \\
\hline
\end{tabular}

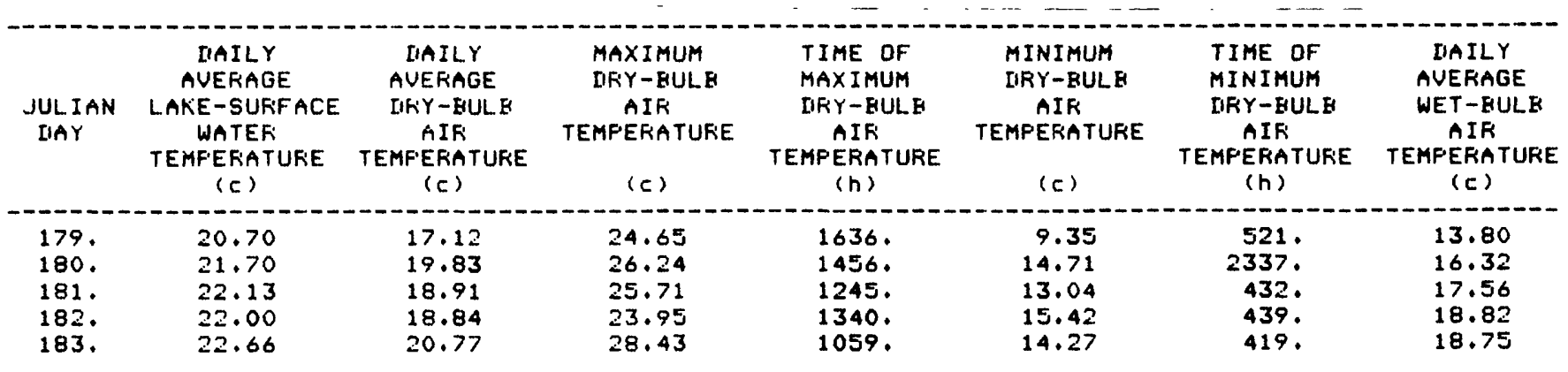

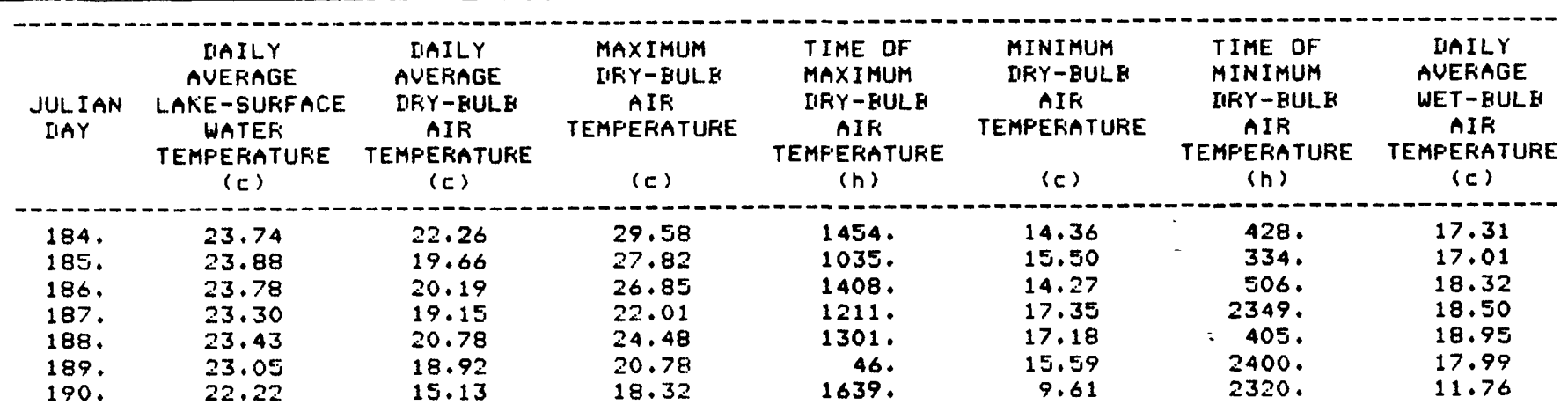

\begin{tabular}{|c|c|c|c|c|c|c|c|}
\hline & $\begin{array}{l}\text { IIAIL.Y } \\
\text { AUERIAGE }\end{array}$ & $\begin{array}{l}\text { IIAILY } \\
\text { AUERIAGE }\end{array}$ & $\begin{array}{l}\text { MAXIMUM } \\
\text { IIFY-BUL.E }\end{array}$ & $\begin{array}{l}\text { TIME OF } \\
\text { MAXIMUM }\end{array}$ & $\begin{array}{l}\text { MINIMUM } \\
\text { IRY-BULE }\end{array}$ & $\begin{array}{l}\text { TIME OF } \\
\text { MINIMUM }\end{array}$ & $\begin{array}{c}\text { IIAILYY } \\
\text { AUERAGE }\end{array}$ \\
\hline $\begin{array}{l}\text { JUL IAN } \\
\text { IIAY }\end{array}$ & $\begin{array}{l}\text { LAKE-SUFIFACE } \\
\text { WATER } \\
\text { TEMFEFATUFE } \\
\text { (C) }\end{array}$ & $\begin{array}{c}\text { IIRY-EULE } \\
\text { AIR } \\
\text { TEMFERATURE } \\
\text { (C) }\end{array}$ & $\begin{array}{c}\text { AIF } \\
\text { TEMFEFIATURE } \\
\text { (C) }\end{array}$ & $\begin{array}{c}\text { IIFYY-EULE } \\
\text { AIF } \\
\text { TEMFERATUFE } \\
(h)\end{array}$ & $\begin{array}{l}\text { AIF } \\
\text { TEMPEFIATURE } \\
\text { (C) }\end{array}$ & $\begin{array}{c}\text { IIRY - EULE } \\
\text { AIF } \\
\text { TEMFERATURE } \\
(h)\end{array}$ & $\begin{array}{l}\text { WET-EULE } \\
\text { AIR } \\
\text { TEMFERATUFE } \\
\text { (C) }\end{array}$ \\
\hline & -ー-ーーーー & $----\infty---r$ & ---- & --------- & $---\infty$ & --------- & --------- \\
\hline $\begin{array}{l}191 . \\
192 . \\
193 .\end{array}$ & $\begin{array}{l}21.68 \\
22.52 \\
22.06\end{array}$ & $\begin{array}{l}14.63 \\
18.46 \\
18.47\end{array}$ & $\begin{array}{l}21 \cdot 57 \\
25.09 \\
22.89\end{array}$ & $\begin{array}{l}1146 . \\
1337 . \\
849 .\end{array}$ & $\begin{array}{r}7.94 \\
12.43 \\
13.22\end{array}$ & $\begin{array}{l}343 . \\
510 \\
328\end{array}$ & $\begin{array}{l}12.74 \\
15.93 \\
17.49\end{array}$ \\
\hline
\end{tabular}

194.

195.

196. 
Table 2. Summary of 1984 data: Raft station temperature--(1) daily average lake surface water temperature, (2) dafly average dry-bulb alr temperature, (3) dafly maximum and minimum dry-bulb alr temperature and the time they occurred, and (4) dafly average wet-bulb alr temperature. (continued)

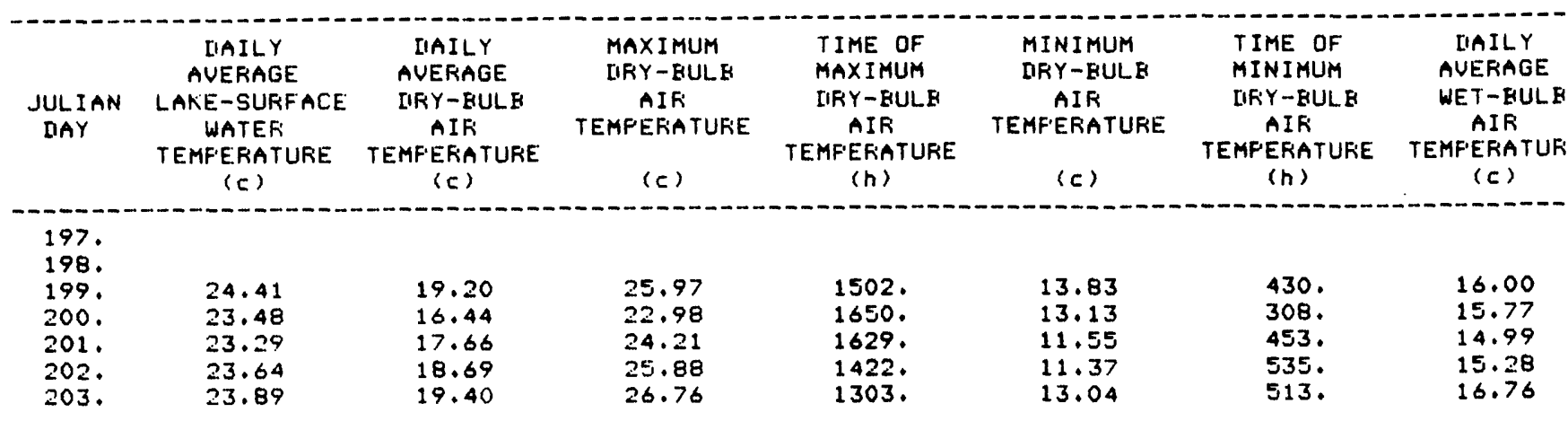

\begin{tabular}{|c|c|c|c|c|c|c|c|}
\hline JULIAN & $\begin{array}{c}\text { IIAILY } \\
\text { AUERAGE } \\
\text { LAKE-SURFACE } \\
\text { WATER } \\
\text { TEMFERATURE } \\
\text { (C) }\end{array}$ & $\begin{array}{l}\text { IIAILY } \\
\text { AUERAGE } \\
\text { IIRY-BULLE } \\
\text { AIF } \\
\text { TEMFERATURE } \\
\text { (C) }\end{array}$ & $\begin{array}{c}\text { MAXIMUM } \\
\text { LIRY - BULE } \\
\text { AIR } \\
\text { TEMFEFATURE } \\
\text { (C) }\end{array}$ & $\begin{array}{l}\text { TIME OF } \\
\text { MAXIMUM } \\
\text { IIRY-BULE } \\
\text { AIR } \\
\text { TEMFERATURE } \\
\text { (h) }\end{array}$ & $\begin{array}{c}\text { MINIMUM } \\
\text { IIRY - BULL } \\
\text { AIR } \\
\text { TEMFERATURE } \\
\text { (C) }\end{array}$ & $\begin{array}{l}\text { TIME OF } \\
\text { MINIMUM } \\
\text { IIRY-EULE } \\
\text { AIR } \\
\text { TEMPERATURE } \\
\text { (h) }\end{array}$ & $\begin{array}{l}\text { DAILY } \\
\text { AUERAGE } \\
\text { WET-EULE } \\
\text { AIR } \\
\text { TEMFERATURE } \\
\text { (C) }\end{array}$ \\
\hline $\begin{array}{l}204 . \\
205 . \\
206 . \\
207 . \\
208 . \\
209 . \\
210 .\end{array}$ & $\begin{array}{l}24.44 \\
24.54 \\
24.22 \\
23.44 \\
23.20 \\
22.53 \\
22.21\end{array}$ & $\begin{array}{l}20.83 \\
21.88 \\
22.88 \\
17.02 \\
16.52 \\
14.62 \\
16.52\end{array}$ & $\begin{array}{l}29.14 \\
27.73 \\
25.97 \\
21.57 \\
23.51 \\
16.91 \\
22.54\end{array}$ & $\begin{array}{l}1350^{\circ} \\
1729^{\circ} \\
1047^{\circ} \\
1652^{\circ} \\
13177^{\circ} \\
10440^{\circ} \\
1628^{\circ}\end{array}$ & $\begin{array}{r}14.27 \\
15.59 \\
14.98 \\
10.40 \\
8.38 \\
12.95 \\
12.60\end{array}$ & $\begin{array}{r}350 . \\
452 . \\
2359 . \\
2351 . \\
435 . \\
2400 . \\
2337 .\end{array}$ & $\begin{array}{l}17.51 \\
18.57 \\
16.44 \\
12.05 \\
13.65 \\
13.86 \\
14.17\end{array}$ \\
\hline
\end{tabular}

\begin{tabular}{|c|c|c|c|c|c|c|c|}
\hline $\begin{array}{l}\text { JUL I AN } \\
\text { IIAY }\end{array}$ & $\begin{array}{c}\text { IIAILY } \\
\text { AUERAGE } \\
\text { LAKE-SURFACE } \\
\text { WATEF } \\
\text { TEMFERATUFE } \\
\text { (C) }\end{array}$ & $\begin{array}{l}\text { IIAILY } \\
\text { AUERAGE } \\
\text { IIRY-EULE } \\
\text { AIR } \\
\text { TEMFERATURE } \\
\text { (C) }\end{array}$ & $\begin{array}{c}\text { MAXIMUM } \\
\text { IIFY - BULLE } \\
\text { AIF } \\
\text { TEMFERATURE } \\
\text { (C) }\end{array}$ & $\begin{array}{c}\text { TIME OF } \\
\text { MAXIMUM } \\
\text { IIRY-EULE } \\
\text { AIR } \\
\text { TEMF'ERATUFE } \\
\text { (h) }\end{array}$ & $\begin{array}{c}\text { MINIMUM } \\
\text { IIRY-EULE } \\
\text { AIR } \\
\text { TEMFERATURE } \\
\text { (C) }\end{array}$ & $\begin{array}{c}\text { TIME OF } \\
\text { MINIMUM } \\
\text { IIRY - EULLE } \\
\text { AIR } \\
\text { TEMFERATURE } \\
(h)\end{array}$ & $\begin{array}{l}\text { IIAILY } \\
\text { AUERAGE } \\
\text { WET-BULE } \\
\text { AIR } \\
\text { TEMFERATURE } \\
\text { (C) }\end{array}$ \\
\hline $\begin{array}{l}211 . \\
212 . \\
213 . \\
214 . \\
215 . \\
216 . \\
217 . \\
218 .\end{array}$ & $\begin{array}{l}22.79 \\
23.35 \\
23.82 \\
23.47 \\
23.79 \\
24.10 \\
24.27 \\
24.90\end{array}$ & $\begin{array}{l}17.93 \\
18.80 \\
20.94 \\
19.71 \\
21.65 \\
21.00 \\
21.60 \\
21.99\end{array}$ & $\begin{array}{l}26.68 \\
27.47 \\
28.00 \\
25.27 \\
28.61 \\
27.91 \\
29.05 \\
29.75\end{array}$ & $\begin{array}{l}16000^{\circ} \\
1254^{\circ} \\
1611^{\circ} \\
1159^{\circ} \\
1333^{\circ} \\
1316^{\circ} \\
1427^{\circ} \\
1419^{\circ}\end{array}$ & $\begin{array}{l}10.67 \\
11.46 \\
14.54 \\
15.59 \\
16.65 \\
15.50 \\
15.15 \\
15.06\end{array}$ & $\begin{array}{l}448 . \\
436 . \\
528 . \\
452 . \\
2359^{\circ} \\
406 . \\
445 . \\
419 .\end{array}$ & $\begin{array}{l}14.27 \\
15.34 \\
17.05 \\
17.32 \\
18.37 \\
17.85 \\
17.87 \\
18.49\end{array}$ \\
\hline
\end{tabular}


Table 2. Summary of 1984 data: Raft station temperature--(1) dafly average lake surface water temperature, (2) dally average dry-bulb air temperature, (3) daily maximum and minimum dry-bulb air temperature and the time they occurred, and (4) dafly average wet-bulb air temperature. (continued)

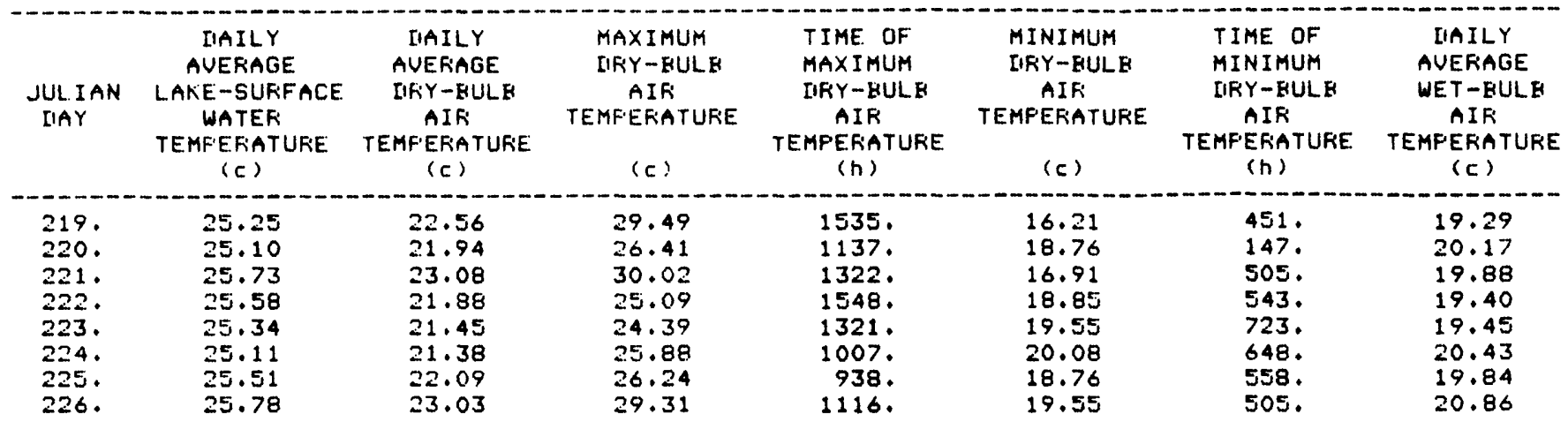

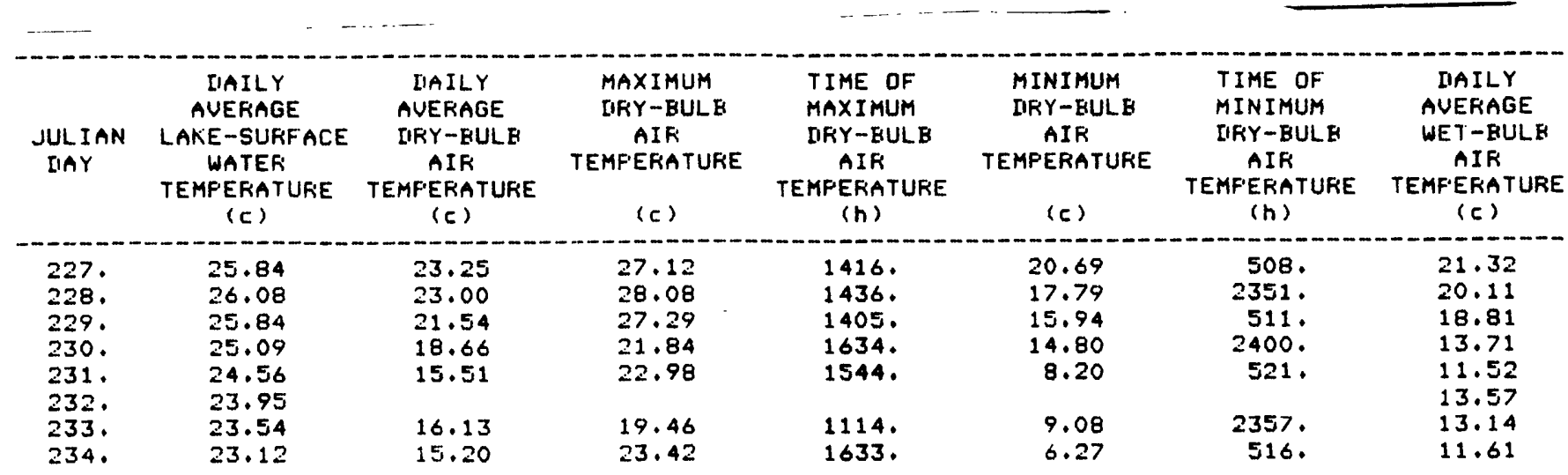

\begin{tabular}{|c|c|c|c|c|c|c|c|}
\hline $\begin{array}{l}\text { JULI I AN } \\
\text { IIAY }\end{array}$ & $\begin{array}{c}\text { IUAILY } \\
\text { AUERAGE } \\
\text { LAKE-SURFACE } \\
\text { WATER } \\
\text { TEMFERATURE } \\
\text { (C) }\end{array}$ & $\begin{array}{l}\text { IIAILY } \\
\text { AUERAGE } \\
\text { IIRY - BULE } \\
\text { AIF } \\
\text { TEMFERATURE } \\
\text { (C) }\end{array}$ & $\begin{array}{l}\text { MAXIMUM } \\
\text { IIRY - BULE } \\
\text { AIF } \\
\text { TEMFERATUFE } \\
\text { (C) }\end{array}$ & $\begin{array}{l}\text { TIME OF } \\
\text { MAXIMUM } \\
\text { IIRY - EULE } \\
\text { AIR } \\
\text { TEMFERATURE } \\
\text { (h) }\end{array}$ & $\begin{array}{l}\text { MINIMUM } \\
\text { IIRY-EULE } \\
\text { AIR } \\
\text { TEMPERATURE } \\
\text { (C) }\end{array}$ & $\begin{array}{c}\text { TIME OF } \\
\text { MINIMUM } \\
\text { IIRY - BULE } \\
\text { AIR } \\
\text { TEMFERATURE } \\
(h)\end{array}$ & $\begin{array}{l}\text { IIAILY } \\
\text { AUERAGE } \\
\text { WET-BULE } \\
\text { AIR } \\
\text { TEMPEFATURE } \\
\text { (C) }\end{array}$ \\
\hline 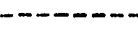 & $---m-m$ & 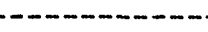 & $----m-$ & - & ---- & $-------n---$ & $\therefore------n$ \\
\hline $\begin{array}{l}235 . \\
236 . \\
237 . \\
238 . \\
239 . \\
240 . \\
241 . \\
242 . \\
243 . \\
244 . \\
245 .\end{array}$ & $\begin{array}{l}23.44 \\
23.12 \\
22.80 \\
22.64 \\
22.71 \\
22.78 \\
23.06 \\
23.16 \\
23.17 \\
22.96 \\
22.40\end{array}$ & $\begin{array}{l}18.77 \\
17.84 \\
16.97 \\
15.94 \\
16.27 \\
17.39 \\
19.03 \\
20.54 \\
21.87 \\
19.50 \\
15.59\end{array}$ & $\begin{array}{l}26.76 \\
24.21 \\
21.93 \\
22.28 \\
24.21 \\
26.41 \\
27.12 \\
27.29 \\
27.91 \\
25.71 \\
20.61\end{array}$ & $\begin{array}{l}1457 . \\
1432 . \\
1353 . \\
1502 . \\
1538 . \\
1545 . \\
1439 . \\
1358 . \\
1337 . \\
1235 . \\
1153 .\end{array}$ & $\begin{array}{r}11.02 \\
14.71 \\
12.60 \\
10.05 \\
8.03 \\
9.88 \\
10.58 \\
15.24 \\
18.67 \\
13.22 \\
10.31\end{array}$ & $\begin{array}{r}516 . \\
2350 . \\
2400 . \\
528 . \\
503 . \\
457 . \\
546 . \\
530 . \\
530 . \\
2348 . \\
539 .\end{array}$ & $\begin{array}{l}14.80 \\
16.90 \\
14.86 \\
12.43 \\
13.06 \\
14.22 \\
15.72 \\
18.84 \\
20.03 \\
16.69 \\
12.31\end{array}$ \\
\hline
\end{tabular}


Table 2. Summary of 1984 data: Raft station temperature--(1) daily average lake surface water temperature, (2) dally average dry-bulb air temperature, (3) daily maximum and minfmum dry-bulb air temperature and the time they occurred, and (4) dally average wet-bulb afr temperature. (continued)

\begin{tabular}{|c|c|c|c|c|c|c|c|}
\hline $\begin{array}{l}\text { JUL I AN } \\
\text { IIAY }\end{array}$ & $\begin{array}{l}\text { IIAILY } \\
\text { AUEFIAGE } \\
\text { LAKE-SUFFACE. } \\
\text { WATEF } \\
\text { TEMFEFIATUFE } \\
\text { (C) }\end{array}$ & $\begin{array}{l}\text { IIAILY } \\
\text { AUEFIAGE } \\
\text { IIFY - BULE } \\
\text { AIF } \\
\text { TEMF'EFATURE. } \\
\text { (C) }\end{array}$ & $\begin{array}{l}\text { MAXIMUM } \\
\text { IIFY - EULE } \\
\text { AIF } \\
\text { TEMFEFITURE } \\
\text { (C) }\end{array}$ & $\begin{array}{l}\text { TIME OF } \\
\text { MAXIMUM } \\
\text { IIFY - EULE } \\
\text { AIF } \\
\text { TEMF'EFATUFIE } \\
\text { ( } h \text { ) }\end{array}$ & $\begin{array}{l}\text { MINIMUM } \\
\text { LIRY - BULE } \\
\text { AIF } \\
\text { TEMF'EFATURE } \\
\text { (C) }\end{array}$ & $\begin{array}{l}\text { TIME OF } \\
\text { MINIMUM } \\
\text { IIRY - BULE } \\
\text { AIF } \\
\text { TEMFERATUFE } \\
\text { ( } h \text { ) }\end{array}$ & $\begin{array}{l}\text { IIAIL.Y } \\
\text { AUERAGE } \\
\text { WET - BUL.E } \\
\text { AIF } \\
\text { TEMFEFIATUFE } \\
\text { (C) }\end{array}$ \\
\hline $\begin{array}{l}246 . \\
247 . \\
248 . \\
249 . \\
250 . \\
251 . \\
252 . \\
253 . \\
254 .\end{array}$ & $\begin{array}{l}21.71 \\
21.11 \\
20.68 \\
20.36 \\
19.80 \\
19.42 \\
19.40 \\
19.71 \\
19.71\end{array}$ & $\begin{array}{r}14.50 \\
12.22 \\
13.88 \\
12.36 \\
9.28 \\
9.95 \\
12.76 \\
15.09 \\
16.28\end{array}$ & $\begin{array}{l}19.64 \\
16.03 \\
17.35 \\
17.97 \\
14.62 \\
18.50 \\
22.19 \\
24.48 \\
23.69\end{array}$ & $\begin{array}{l}1513 . \\
1413 . \\
1336 \\
1154 \\
1446 \\
1420 \\
1555 . \\
1444 \\
1347\end{array}$ & $\begin{array}{r}8.73 \\
9.08 \\
11.55 \\
6.80 \\
3.19 \\
1.17 \\
4.07 \\
6.89 \\
7.51\end{array}$ & $\begin{array}{l}458 . \\
400 . \\
341 . \\
528 . \\
531 . \\
526 . \\
536 . \\
603 . \\
608 .\end{array}$ & $\begin{array}{r}10.61 \\
10.97 \\
12.48 \\
9.43 \\
6.65 \\
7.80 \\
12.75 \\
15.07 \\
14.17\end{array}$ \\
\hline
\end{tabular}

\begin{tabular}{|c|c|c|c|c|c|c|c|}
\hline $\begin{array}{l}\text { JUL. I AN } \\
\text { IIAY }\end{array}$ & $\begin{array}{c}\text { IIAILY } \\
\text { AUEFIAGE } \\
\text { LAKE-SUFIFACE } \\
\text { WATEF } \\
\text { TEMF'EFIATUFE } \\
\text { (C) }\end{array}$ & $\begin{array}{l}\text { DAILY } \\
\text { AUERAGE } \\
\text { IIRY - BULE } \\
\text { AIF } \\
\text { TEMFEFATURE } \\
\text { (C) }\end{array}$ & $\begin{array}{l}\text { MAXIMUM } \\
\text { CIRY - BULE } \\
\text { AIR } \\
\text { TEMFERATUFE } \\
\text { (C) }\end{array}$ & $\begin{array}{l}\text { TIME OF } \\
\text { MAXIMUM } \\
\text { IIFY - BULLE } \\
\text { AIR } \\
\text { TEMFEFITURE } \\
\text { ( } h \text { ) }\end{array}$ & $\begin{array}{l}\text { MINIMUM } \\
\text { IIFY - BULE } \\
\text { AIF } \\
\text { TEMFERATURE } \\
\text { (C) }\end{array}$ & $\begin{array}{l}\text { TIME OF } \\
\text { MINIMUH } \\
\text { [IRY-BULE } \\
\text { AIF } \\
\text { TEMPERATURE } \\
\text { (h) }\end{array}$ & $\begin{array}{l}\text { IIAILY } \\
\text { AUERAGE } \\
\text { WET-BULE } \\
\text { AIR } \\
\text { TEMPERATURE } \\
\text { (C) }\end{array}$ \\
\hline $\begin{array}{l}255 . \\
256 . \\
257 . \\
258 . \\
259 . \\
260 . \\
261 . \\
262 .\end{array}$ & $\begin{array}{l}19.83 \\
19.22 \\
18.91 \\
19.03 \\
18.57 \\
17.96 \\
17.69 \\
17.82\end{array}$ & $\begin{array}{r}17.90 \\
14.23 \\
12.41 \\
16.70 \\
10.00 \\
8.65 \\
9.37 \\
10.68\end{array}$ & $\begin{array}{l}22.81 \\
17.79 \\
17.35 \\
21.75 \\
13.75 \\
13.31 \\
17.97 \\
20.69\end{array}$ & $\begin{array}{r}1116 . \\
1355^{\circ} \\
1038 . \\
1419 . \\
13^{\circ} . \\
1455^{\circ} \\
1609^{\circ} \\
1519 .\end{array}$ & $\begin{array}{r}14.36 \\
8.38 \\
7.42 \\
12.78 \\
7.33 \\
3.99 \\
3.72 \\
2.32\end{array}$ & $\begin{array}{r}2125 . \\
2356 . \\
240 . \\
2341 . \\
2159 . \\
630 . \\
554 . \\
551 .\end{array}$ & 16.36 \\
\hline
\end{tabular}

\begin{tabular}{|c|c|c|c|c|c|c|c|}
\hline $\begin{array}{l}\text { JULIAN } \\
\text { IIAY }\end{array}$ & $\begin{array}{l}\text { IIAILY } \\
\text { AUERAGE } \\
\text { LAKE-SUFFACE } \\
\text { WATER } \\
\text { TEMFERATURE } \\
\text { (C) }\end{array}$ & $\begin{array}{l}\text { IIAILY } \\
\text { AUERAGE } \\
\text { DRY - BULE } \\
\text { AIR } \\
\text { TEMFERATURE } \\
\text { (C) }\end{array}$ & $\begin{array}{l}\text { MAXIMUM } \\
\text { IIRY - BULE } \\
\text { AIR } \\
\text { TEMPERATURE } \\
\text { (E) }\end{array}$ & $\begin{array}{l}\text { TIME OF } \\
\text { MAXIMUM } \\
\text { IIRY-BULE } \\
\text { AIF } \\
\text { TEMPEFIATURE } \\
\text { (h) }\end{array}$ & $\begin{array}{l}\text { MINIMUM - } \\
\text { IRY-BULE } \\
\text { AIF } \\
\text { TEMPERATURE : } \\
\text { (C) }\end{array}$ & $\begin{array}{l}\text { TIME OF } \\
\text { MINIMUM } \\
\text { IRY-EULE } \\
\text { AIR } \\
\text { TEMPERATURE } \\
\text { (h) }\end{array}$ & $\begin{array}{l}\text { IIAILY } \\
\text { AUERAGE } \\
\text { WET - EULE } \\
\text { AIR } \\
\text { TEMFERATURE } \\
\text { (C) }\end{array}$ \\
\hline & $--\infty-\infty$ & $---m$ & $------\infty$ & $--m-\infty$ & $-\infty---\infty$ & $-----m$ & $--------\infty$ \\
\hline $\begin{array}{l}263 . \\
264 . \\
265 . \\
266 . \\
267 .\end{array}$ & $\begin{array}{l}17.69 \\
17.83 \\
17.55 \\
17.53 \\
18.23\end{array}$ & $\begin{array}{r}12.07 \\
15.13 \\
11.38 \\
9.97 \\
15.95\end{array}$ & $\begin{array}{l}21.31 \\
26.06 \\
15.42 \\
20.17 \\
26.41\end{array}$ & $\begin{array}{l}1424 . \\
1517 . \\
1519 . \\
1551 . \\
1505 .\end{array}$ & $\begin{array}{l}3.72 \\
7.59 \\
4.34 \\
1.08 \\
5.31\end{array}$ & $\begin{array}{r}553 . \\
324 . \\
2358 . \\
604 . \\
510 .\end{array}$ & $\begin{array}{r}9.97 \\
12.31 \\
7.87 \\
7.21 \\
13.44\end{array}$ \\
\hline
\end{tabular}


Table 2. Summary of 1984 data: Raft station temperature--(1) dally average lake surface water temperature, (2) dally average dry-bulb alr temperature, (3) dafly maximum and minimum dry-bulb alr temperature and the time they occurred, and (4) dafly average wet-bulb air temperature. (continued)

\begin{tabular}{|c|c|c|c|c|c|c|c|}
\hline $\begin{array}{l}\text { JUL_ IAN } \\
\text { IIAYY }\end{array}$ & $\begin{array}{c}\text { IIAILY } \\
\text { AUEFIAGE } \\
\text { LAKE-SUFFACE } \\
\text { WATER } \\
\text { TEMFEFATUFE } \\
\text { (C) }\end{array}$ & $\begin{array}{l}\text { IIAILY } \\
\text { AUERAGE } \\
\text { LIFY-EULE } \\
\text { AIR } \\
\text { TEMFERATURE } \\
\text { (C) }\end{array}$ & $\begin{array}{c}\text { MAXIMUM } \\
\text { IRY-EULE } \\
\text { AIF } \\
\text { TEMFERATURE } \\
\text { (C) }\end{array}$ & $\begin{array}{l}\text { TIME OF } \\
\text { MAXIMUM } \\
\text { IIRY-EIULE } \\
\text { AIR } \\
\text { TEMFEEATURE } \\
\text { ( } h \text { ) }\end{array}$ & $\begin{array}{c}\text { MINIMUM } \\
\text { LIRY-EULE } \\
\text { AIF } \\
\text { TEMFERATUFE } \\
\text { (C) }\end{array}$ & $\begin{array}{c}\text { TIME OF } \\
\text { MINIMUM } \\
\text { IIFY - EULE } \\
\text { AIR } \\
\text { TEMFERATURE } \\
\text { ( } h \text { ) }\end{array}$ & $\begin{array}{l}\text { IIAILY } \\
\text { AUERAGE } \\
\text { WET-EULE } \\
\text { AIF } \\
\text { TEMFERATURE } \\
\text { (C) }\end{array}$ \\
\hline $\begin{array}{l}268 . \\
269 . \\
270 . \\
271 . \\
272 . \\
273 . \\
274 . \\
275 . \\
270^{\circ} . \\
277 . \\
278 . \\
279 . \\
280 . \\
281 .\end{array}$ & $\begin{array}{l}19.06 \\
19.13 \\
18.58 \\
17.05 \\
16.82 \\
16.51 \\
16.31 \\
15.85 \\
15.32 \\
14.68 \\
14.34 \\
13.86 \\
13.15 \\
13.16\end{array}$ & $\begin{array}{r}19.58 \\
17.86 \\
13.15 \\
6.67 \\
8.22 \\
7.98 \\
8.15 \\
8.27 \\
5.88 \\
7.34 \\
7.01 \\
4.03 \\
3.78 \\
6.02\end{array}$ & $\begin{array}{r}25.44 \\
24.65 \\
21.49 \\
12.07 \\
14.89 \\
13.92 \\
16.82 \\
12.60 \\
8.29 \\
10.49 \\
11.46 \\
7.59 \\
9.00 \\
18.41\end{array}$ & $\begin{array}{r}1547 . \\
1548 . \\
724 . \\
1520^{\circ} \\
1630 . \\
1640 . \\
1318 . \\
1339 . \\
40 . \\
2400 . \\
1543 . \\
311 . \\
1558 . \\
1502 .\end{array}$ & $\begin{array}{r}13.83 \\
12.78 \\
5.74 \\
3.02 \\
2.58 \\
3.19 \\
.91 \\
4.87 \\
4.43 \\
2.76 \\
2.40 \\
-.15 \\
-1.20 \\
-3.22\end{array}$ & $\begin{array}{r}2400 . \\
515 . \\
2359 . \\
604 . \\
530 . \\
2341 . \\
618 . \\
115 . \\
2241 . \\
619 . \\
2340 . \\
1952 . \\
2352 . \\
524 .\end{array}$ & $\begin{array}{r}15.69 \\
16.06 \\
10.30 \\
2.98 \\
5.26 \\
6.36 \\
5.95 \\
7.15 \\
4.59 \\
6.28 \\
3.96 \\
.71 \\
-.26 \\
2.27\end{array}$ \\
\hline
\end{tabular}

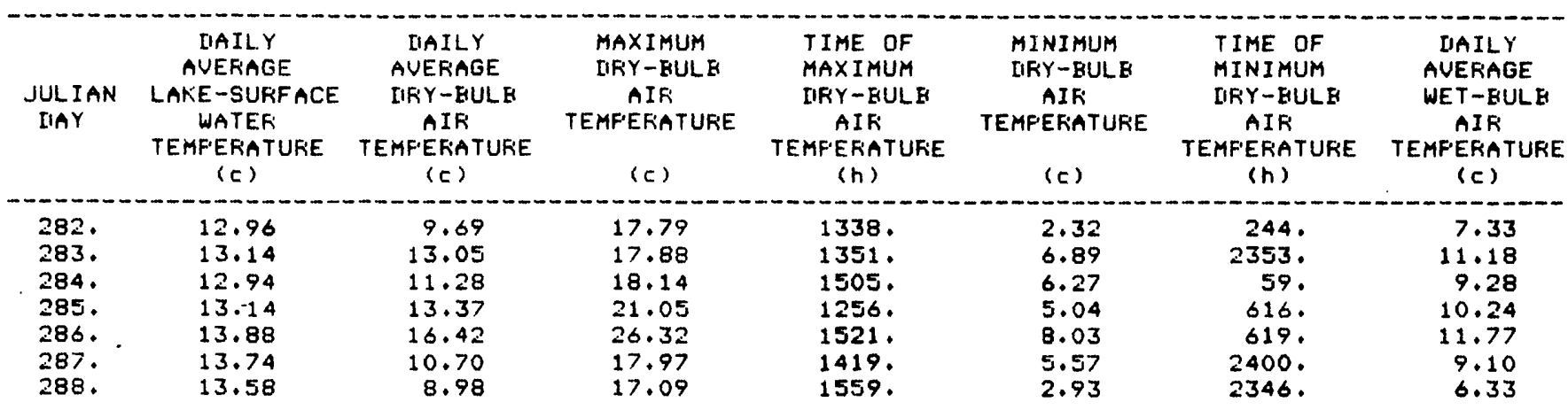

\begin{tabular}{|c|c|c|c|c|c|c|c|}
\hline $\begin{array}{l}\text { JUL I IAN } \\
\text { IIAY }\end{array}$ & $\begin{array}{c}\text { IIAILY } \\
\text { AUERAGE } \\
\text { LAKE-SURFACE } \\
\text { WATER } \\
\text { TEMFERATURE } \\
\text { (C) }\end{array}$ & $\begin{array}{l}\text { DAILY } \\
\text { AUERAGE } \\
\text { IIRY - EULE } \\
\text { AIR } \\
\text { TEMFERATURE } \\
\text { (C) }\end{array}$ & $\begin{array}{l}\text { MAXIMUM } \\
\text { INRY-EULE } \\
\text { AIR } \\
\text { TEMFERATURE } \\
\text { (C) }\end{array}$ & $\begin{array}{c}\text { TIME OF } \\
\text { MAXIMUM } \\
\text { IIRY - BULE } \\
\text { AIR } \\
\text { TEMFERATURE } \\
\text { (h) }\end{array}$ & $\begin{array}{c}\text { MINIMUM } \\
\text { IRYY-EULE } \\
\text { AIR } \\
\text { TEMF'ERATURE } \\
\text { (C) }\end{array}$ & $\begin{array}{l}\text { TIME OF } \\
\text { MINIMUM } \\
\text { IRY-EULE } \\
\text { AIR } \\
\text { TEMFERATURE } \\
\text { (h) }\end{array}$ & $\begin{array}{l}\text { IIAILY } \\
\text { AUERAGE } \\
\text { WET-EULE } \\
\text { AIR } \\
\text { TEMPERATURE } \\
\text { (C) }\end{array}$ \\
\hline $\begin{array}{l}289 . \\
290 . \\
291 . \\
292 . \\
293 . \\
294 . \\
295^{\circ} . \\
296 . \\
297 . \\
298 .\end{array}$ & $\begin{array}{l}13.55 \\
13.52 \\
13.40 \\
13.37 \\
13.66 \\
13.90 \\
13.15 \\
13.13 \\
13.14 \\
12.92\end{array}$ & $\begin{array}{r}8.90 \\
10.86 \\
8.26 \\
8.37 \\
11.00 \\
13.54 \\
8.37 \\
12.68 \\
10.32 \\
7.41\end{array}$ & $\begin{array}{l}21.05 \\
21.40 \\
17.70 \\
19.46 \\
22.28 \\
20.25 \\
10.84 \\
21.13 \\
13.22 \\
13.31\end{array}$ & $\begin{array}{l}1335^{\circ} \\
15270^{\circ} \\
15440^{\circ} \\
1519^{\circ} \\
1440^{\circ} \\
14477^{\circ} \\
12080^{\circ} \\
10400^{\circ} \\
1420 . \\
1543 .\end{array}$ & $\begin{array}{l}-.15 \\
2.76 \\
2.14 \\
.20 \\
.73 \\
6.71 \\
4.95 \\
8.73 \\
6.54 \\
1.79\end{array}$ & $\begin{array}{r}534 . \\
545 . \\
605 . \\
601 . \\
607 \\
2400 \\
554 \\
338 \\
2358 \\
2357\end{array}$ & $\begin{array}{r}5.77 \\
7.95 \\
6.28 \\
5.95 \\
8.23 \\
10.92 \\
7.19 \\
10.90 \\
8.91 \\
5.66\end{array}$ \\
\hline
\end{tabular}


Table 2. Summary of 1984 data: Raft station temperature--(1) dally average lake surface water temperature, (2) dafly average dry-bulb alr temperature, (3) dafly maximum and minimum dry-bulb afr temperature and the time they occurred, and (4) dafly average wet-bulb alr temperature. (continued)

\begin{tabular}{|c|c|c|c|c|c|c|c|}
\hline $\begin{array}{l}\text { JUL I AN } \\
\text { IIAY }\end{array}$ & $\begin{array}{l}\text { IIAILY } \\
\text { AUEFIAGE } \\
\text { LAKE-SUFFFACE } \\
\text { WATEF } \\
\text { TEMFEFATUFE } \\
\text { (C) }\end{array}$ & $\begin{array}{c}\text { IIAILY } \\
\text { AUERAGE } \\
\text { IIFY - BULE } \\
\text { AIF } \\
\text { TEMFERATUFE } \\
\text { (C) }\end{array}$ & $\begin{array}{l}\text { MAXIMUM } \\
\text { IIFY - BULE } \\
\text { AIF } \\
\text { TEMFERATURE } \\
\text { (C) }\end{array}$ & $\begin{array}{c}\text { TIME OF } \\
\text { MAXIMUM } \\
\text { IIRY-BULE } \\
\text { AIF } \\
\text { TEMFERATURE } \\
\text { ( } h)\end{array}$ & $\begin{array}{l}\text { MINIMUM } \\
\text { IIFY-BULE } \\
\text { AIF } \\
\text { TEMPERATUFE } \\
\text { (C) }\end{array}$ & $\begin{array}{l}\text { TIME OF } \\
\text { MINIMUM } \\
\text { IIRY-EUL. } \\
\text { AIF } \\
\text { TEMFERATUFE } \\
\text { ( } h)\end{array}$ & $\begin{array}{l}\text { IIAILY } \\
\text { AUERAGE } \\
\text { WET-BULE } \\
\text { AIR } \\
\text { TEMPERATURE } \\
\text { (C) }\end{array}$ \\
\hline $\begin{array}{l}299 . \\
300 . \\
301 . \\
302 . \\
303 . \\
304 .\end{array}$ & $\begin{array}{l}12.76 \\
12.44 \\
12.35 \\
12.53 \\
12.49 \\
12.19\end{array}$ & $\begin{array}{r}6.43 \\
6.15 \\
7.28 \\
11.18 \\
12.51 \\
4.67\end{array}$ & $\begin{array}{r}14.54 \\
7.42 \\
9.61 \\
17.62 \\
16.74 \\
13.13\end{array}$ & $\begin{array}{r}1517 \\
1423 \\
1420 \\
1502 \\
915 \\
1531\end{array}$ & $\begin{array}{r}-1.11 \\
4.87 \\
4.78 \\
8.03 \\
3.02 \\
-1.64\end{array}$ & $\begin{array}{r}513 . \\
2330 . \\
346 . \\
244 . \\
2354 . \\
616 .\end{array}$ & $\begin{array}{r}4.13 \\
5.71 \\
6.68 \\
10.58 \\
8.83 \\
2.58\end{array}$ \\
\hline
\end{tabular}

\begin{tabular}{|c|c|c|c|c|c|c|c|}
\hline & $\begin{array}{l}\text { IIAILY } \\
\text { AUERAGE }\end{array}$ & $\begin{array}{l}\text { IIAILY } \\
\text { AUERIAGE }\end{array}$ & $\begin{array}{l}\text { MAXIMUM } \\
\text { IIRY-BULE }\end{array}$ & $\begin{array}{l}\text { TIME OF } \\
\text { MAXIMUM }\end{array}$ & $\begin{array}{l}\text { MINIMUM } \\
\text { IIRY - EULE }\end{array}$ & $\begin{array}{l}\text { TIME OF } \\
\text { MINIMUM }\end{array}$ & $\begin{array}{l}\text { IIAILY } \\
\text { AUERAGE }\end{array}$ \\
\hline $\begin{array}{l}\text { JUL I AN } \\
\text { IUAY }\end{array}$ & $\begin{array}{l}\text { LAKE-SURFACE } \\
\text { WATEF } \\
\text { TEMFERATUFE } \\
\text { (C) }\end{array}$ & $\begin{array}{l}\text { IIFY - EULE } \\
\text { AIR } \\
\text { TEMFE.FATUKE } \\
\text { (C) }\end{array}$ & $\begin{array}{l}\text { AIR } \\
\text { TEMFERATURE } \\
\text { (c) }\end{array}$ & $\begin{array}{c}\text { IIRY - HULE } \\
\text { AIR } \\
\text { TEMFEFATUKE } \\
(h)\end{array}$ & $\begin{array}{l}\text { AIF } \\
\text { TEMPERATURE } \\
\text { (C) }\end{array}$ & $\begin{array}{c}\text { IIRY - EULE } \\
\text { AIR } \\
\text { TEMPERATURE } \\
(h)\end{array}$ & $\begin{array}{c}\text { WET-BULE } \\
\text { AIF } \\
\text { TEMFERATUFE } \\
\text { (C) }\end{array}$ \\
\hline $\begin{array}{l}305 . \\
306 \\
307 \\
308 \\
309\end{array}$ & $\begin{array}{l}11.82 \\
11.45 \\
11.24 \\
10.72 \\
10.36\end{array}$ & $\begin{array}{l}4.55 \\
4.09 \\
5.92 \\
1.75 \\
3.27\end{array}$ & $\begin{array}{r}11.37 \\
9.79 \\
10.84 \\
9.00 \\
10.40\end{array}$ & $\begin{array}{r}1316 \\
1502 \\
716 \\
1532 \\
1453\end{array}$ & $\begin{array}{r}-1.55 \\
-4.19 \\
.56 \\
-3.31 \\
-5.68\end{array}$ & $\begin{array}{r}2358 \\
640 \\
2359 \\
2343 \\
614\end{array}$ & $\begin{array}{r}2.26 \\
1.96 \\
3.09 \\
-1.50 \\
1.29\end{array}$ \\
\hline
\end{tabular}

\begin{tabular}{|c|c|c|c|c|c|c|c|}
\hline $\begin{array}{l}\text { JUL I AN } \\
\text { IIAY }\end{array}$ & $\begin{array}{c}\text { IIAILY } \\
\text { AUERAGE } \\
\text { LAKE-SUFFACE } \\
\text { WATER } \\
\text { TEMFEFATUFE } \\
\text { (C) }\end{array}$ & $\begin{array}{c}\text { IIAILY } \\
\text { AUEFIAGE } \\
\text { IIRY - BULE } \\
\text { AIF } \\
\text { TEMF'EFATURE } \\
\text { (C) }\end{array}$ & $\begin{array}{l}\text { MAXIMUM } \\
\text { IIRY - BULE } \\
\text { AIR } \\
\text { TEMFERATURE } \\
\text { (C) }\end{array}$ & $\begin{array}{l}\text { TIME OF } \\
\text { MAXIMUM } \\
\text { IIFY-BULB } \\
\text { AIR } \\
\text { TEMFERATURE } \\
\text { (h) }\end{array}$ & $\begin{array}{l}\text { MINIMUM } \\
\text { DFY - BULE } \\
\text { AIF } \\
\text { TEMFERATURE } \\
\text { (C) }\end{array}$ & $\begin{array}{l}\text { TIME OF } \\
\text { MINIMUM } \\
\text { DRY - BULE } \\
\text { AIR } \\
\text { TEMFEFIATUFE } \\
\text { ( } h \text { ) }\end{array}$ & $\begin{array}{l}\text { IIAILY } \\
\text { AUERAGE } \\
\text { WET - EULE } \\
\text { AIR } \\
\text { TEMFEFATUFE } \\
\text { (C) }\end{array}$ \\
\hline $\begin{array}{l}310 . \\
311 . \\
312 . \\
313 . \\
314 . \\
315 . \\
316 . \\
317 . \\
318 .\end{array}$ & $\begin{array}{r}10.31 \\
10.14 \\
9.65 \\
9.15 \\
8.89 \\
8.85 \\
9.11 \\
9.57 \\
8.67\end{array}$ & $\begin{array}{r}8.24 \\
7.45 \\
.86 \\
-.66 \\
3.06 \\
7.20 \\
9.30 \\
10.16 \\
1.59\end{array}$ & $\begin{array}{r}11.99 \\
10.40 \\
4.78 \\
5.83 \\
8.20 \\
9.17 \\
10.93 \\
15.68 \\
6.01\end{array}$ & $\begin{array}{r}1823 . \\
119 . \\
39 \\
1507 \\
1420 \\
1453 . \\
2233 \\
1332 \\
5 .\end{array}$ & $\begin{array}{r}6.27 \\
4.16 \\
-2.69 \\
-6.74 \\
-.76 \\
3.46 \\
7.24 \\
5.66 \\
-1.37\end{array}$ & $\begin{array}{r}658 . \\
2345 . \\
2400 . \\
642 . \\
4 . \\
1 . \\
632 . \\
2340 . \\
2301 .\end{array}$ & $\begin{array}{r}7.97 \\
4.57 \\
-1.16 \\
-2.05 \\
1.68 \\
7.11 \\
9.09 \\
9.34 \\
.26\end{array}$ \\
\hline
\end{tabular}


Table 2. Summary of 1984 data: Raft station temperature--(1) dally average lake ourface water temperature, (2) dally average dry-bulb alr temperature, (3) datly maximum and minimum dry-bulb afr temperature and the time they occurred, and (4) dally average wet-bulb alr temperature. (continued)

\begin{tabular}{|c|c|c|c|c|c|c|c|}
\hline $\begin{array}{l}\text { JUL I AN } \\
\text { IIAY }\end{array}$ & $\begin{array}{c}\text { IIAILY } \\
\text { AUEFIAGE } \\
\text { LAKE-SUFFACE } \\
\text { WATEF } \\
\text { TEMF'ERATUFE } \\
\text { (C) }\end{array}$ & $\begin{array}{l}\text { DUALY } \\
\text { AUEFAGE } \\
\text { IIRY - BULE } \\
\text { AIFI } \\
\text { TEMFERATUFE } \\
\text { (C) }\end{array}$ & $\begin{array}{l}\text { MAXIMUM } \\
\text { IIFY - BULE } \\
\text { AIR } \\
\text { TEMFEFATUFE } \\
\text { (C) }\end{array}$ & $\begin{array}{c}\text { TIME OF } \\
\text { MAXIMUM } \\
\text { DRY-EULE } \\
\text { AIR } \\
\text { TEMPERATURE } \\
(h)\end{array}$ & $\begin{array}{l}\text { MINIMUM } \\
\text { MRY - BULE } \\
\text { AIR } \\
\text { TEMPERATURE } \\
\text { (C) }\end{array}$ & $\begin{array}{c}\text { TIME OF } \\
\text { MINIMUM } \\
\text { URY - EULE } \\
\text { AIR } \\
\text { TEMPERATURE } \\
\text { (h) }\end{array}$ & $\begin{array}{l}\text { IIAILY } \\
\text { AUEFIAGE } \\
\text { WET-EULE } \\
\text { AIR } \\
\text { TEMPERATUFE } \\
\text { (C) }\end{array}$ \\
\hline $\begin{array}{l}319 . \\
320 . \\
321 . \\
322 . \\
323 .\end{array}$ & $\begin{array}{l}7.87 \\
7.19 \\
6.99 \\
6.71 \\
6.07\end{array}$ & $\begin{array}{r}-.86 \\
1.00 \\
3.55 \\
.20 \\
-1.07\end{array}$ & $\begin{array}{l}1.35 \\
6.36 \\
9.44 \\
3.81 \\
1.43\end{array}$ & $\begin{array}{r}1506 . \\
1226 . \\
1042 . \\
6 . \\
2319 .\end{array}$ & $\begin{array}{r}-4.01 \\
-4.19 \\
-.32 \\
-3.22 \\
-4.28\end{array}$ & $\begin{array}{r}831 . \\
646 . \\
413 . \\
2357 . \\
627 .\end{array}$ & $\begin{array}{l}-.45 \\
1.32\end{array}$ \\
\hline
\end{tabular}

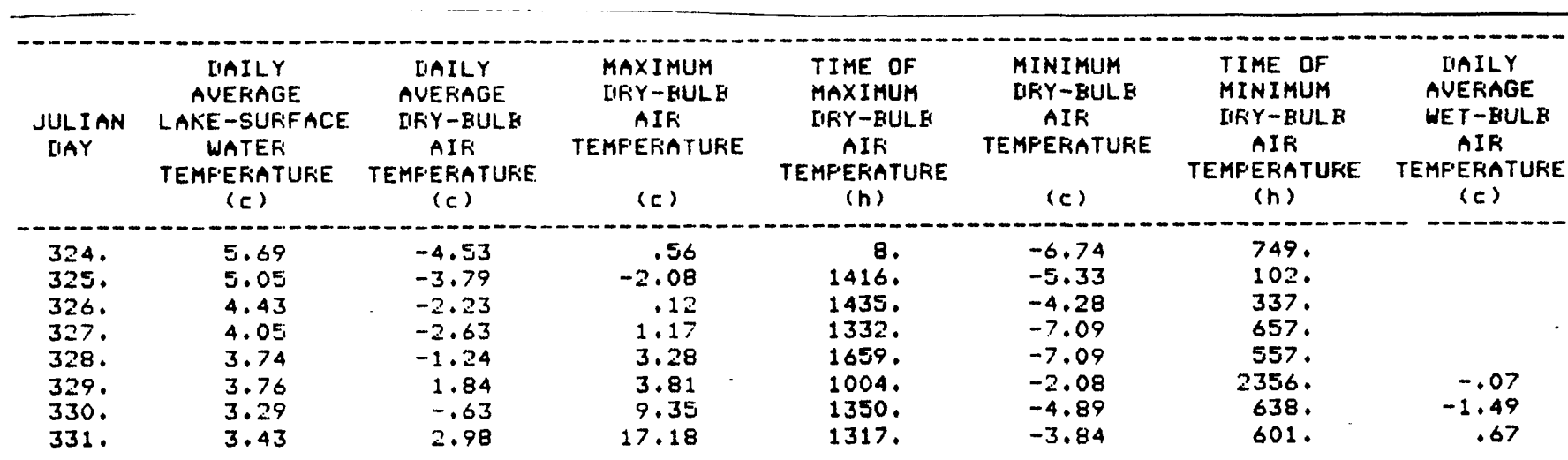

\begin{tabular}{|c|c|c|c|c|c|c|c|}
\hline $\begin{array}{l}\text { JUL I AN } \\
\text { IIAY }\end{array}$ & $\begin{array}{l}\text { DIAILY } \\
\text { AUEFIAGE } \\
\text { LAKE-SUFIFACE } \\
\text { WATER } \\
\text { TEMF'EFIATUFE } \\
\text { (C) }\end{array}$ & $\begin{array}{l}\text { DAILY } \\
\text { AUERAGE } \\
\text { IIRY - BULE } \\
\text { AIF } \\
\text { TEMFERATURE } \\
\text { (C) }\end{array}$ & $\begin{array}{l}\text { MAXIMUM } \\
\text { LIFY - BULE } \\
\text { AIR } \\
\text { TEMFERATUFE } \\
\text { (C) }\end{array}$ & $\begin{array}{c}\text { TIME OF } \\
\text { MAXIHUM } \\
\text { IIRY-BULE } \\
\text { AIR } \\
\text { TEMPERATURE } \\
\text { (h) }\end{array}$ & $\begin{array}{l}\text { MINIMUM } \\
\text { IRY - EULE } \\
\text { AIR } \\
\text { TEMPERATURE } \\
\text { (C) }\end{array}$ & $\begin{array}{l}\text { TIME OF } \\
\text { MINIMUM } \\
\text { IIRY-EULE } \\
\text { AIR } \\
\text { TEMPERATURE } \\
\text { (h) }\end{array}$ & $\begin{array}{l}\text { IIAILY } \\
\text { AUERAGE } \\
\text { WET-EULE } \\
\text { AIR } \\
\text { TEMPERATURE } \\
\text { (C) }\end{array}$ \\
\hline $\begin{array}{l}332 . \\
333 . \\
334 . \\
335 .\end{array}$ & $\begin{array}{l}3.42 \\
3.47 \\
4.21 \\
3.43\end{array}$ & $\begin{array}{l}1.59 \\
4.74 \\
9.45 \\
2.65\end{array}$ & $\begin{array}{r}13.75 \\
11.37 \\
12.95 \\
6.71\end{array}$ & $\begin{array}{r}1350 . \\
1953 . \\
748 . \\
1417 .\end{array}$ & $\begin{array}{r}-4.36 \\
-2.69 \\
4.07 \\
-1.37\end{array}$ & $\begin{array}{r}649 . \\
541 . \\
2255 . \\
2009 .\end{array}$ & $\begin{array}{l}-.04 \\
3.79 \\
8.40 \\
2.02\end{array}$ \\
\hline
\end{tabular}


Table 3. Summary of 1984 data: Raft station wind speed--(1) datly average wind speed at 1,2 and 3 meters above lake surface, (2) daily maximum and mintmum wind speed at the level and the time thev occurred.

$[\mathrm{m} / \mathrm{h}$, miles per hour; $h$, hour; blank, no data]

\begin{tabular}{|c|c|c|c|c|c|c|}
\hline $\begin{array}{c}\text { HEIGHT OF } \\
\text { MEASUREMENT } \\
\text { AEOUE LAKE } \\
\text { SURFACE } \\
(m)\end{array}$ & $\begin{array}{l}\text { JUL I AN } \\
\text { IIAY }\end{array}$ & $\begin{array}{l}\text { IIAILY } \\
\text { AUERAGE } \\
\text { WINII } \\
\text { SFEEI } \\
(m I / h)\end{array}$ & $\begin{array}{l}\text { IIAILY } \\
\text { MAXIMUM } \\
\text { WINI } \\
\text { SFEEI } \\
(m 1 / h)\end{array}$ & $\begin{array}{l}\text { TIME OF } \\
\text { MAXIMUM } \\
\text { WINI } \\
\text { SFEEII } \\
(h)\end{array}$ & $\begin{array}{l}\text { IIAILY } \\
\text { MINIMUM } \\
\text { WINI } \\
\text { SFEEI } \\
(m 1 / h)\end{array}$ & $\begin{array}{l}\text { TIME OF } \\
\text { MINIMUM } \\
\text { WINI } \\
\text { SF'EEI } \\
(h)\end{array}$ \\
\hline 1 & $\begin{array}{l}114 \\
115 \\
116 \\
117 \\
118 \\
119 \\
120 \\
121 \\
122 \\
123 \\
124 \\
125 \\
126 \\
127\end{array}$ & $\begin{array}{l}2.538 \\
3.893 \\
4.207 \\
5.134 \\
2.289 \\
2.104 \\
1.545 \\
3.160 \\
5.591 \\
4.586 \\
2.674 \\
2.520 \\
5.009 \\
3.730\end{array}$ & $\begin{array}{r}11.29 \\
19.92 \\
18.37 \\
21.31 \\
17.58 \\
12.62 \\
8.18 \\
19.50 \\
23.04 \\
19.13 \\
14.04 \\
26.35 \\
26.18 \\
25.07\end{array}$ & $\begin{array}{r}1257 \\
1704 \\
1411 \\
1542 \\
1354 \\
1433 \\
1346 \\
1546 \\
1152 \\
1226 \\
1349 \\
913 \\
803 \\
1202\end{array}$ & $\begin{array}{r}0.571 \\
.571 \\
.571 \\
.571 \\
.571 \\
.600 \\
.571 \\
.571 \\
.571 \\
.600 \\
.571 \\
.571 \\
.600 \\
.571\end{array}$ & $\begin{array}{r}47 \\
229 \\
56 \\
546 \\
2137 \\
2356 \\
2016 \\
1126 \\
2159 \\
2400 \\
2027 \\
1939 \\
2358 \\
2319\end{array}$ \\
\hline 2 & $\begin{array}{l}114 \\
115 \\
116 \\
117 \\
118 \\
119 \\
120 \\
121 \\
122 \\
123 \\
124 \\
125 \\
126 \\
127\end{array}$ & $\begin{array}{l}3.067 \\
4.427 \\
4.909 \\
5.965 \\
3.195 \\
2.906 \\
2.381 \\
4.110 \\
6.398 \\
5.403 \\
3.541 \\
3.522 \\
5.880 \\
4.563\end{array}$ & $\begin{array}{r}11.97 \\
20.91 \\
19.05 \\
26.07 \\
18.71 \\
13.30 \\
9.28 \\
19.53 \\
25.64 \\
20.97 \\
15.14 \\
22.90 \\
27.59 \\
29.49\end{array}$ & $\begin{array}{r}1333 \\
1242 \\
2105 \\
1407 \\
1354 \\
1433 \\
1523 \\
1232 \\
1513 \\
653 \\
1131 \\
913 \\
803 \\
1202\end{array}$ & $\begin{array}{l}.571 \\
.571 \\
.571 \\
.571 \\
.571 \\
.571 \\
.571 \\
.600 \\
.515 \\
.600 \\
.571 \\
.600 \\
.600 \\
.571\end{array}$ & $\begin{array}{r}1945 \\
229 \\
56 \\
458 \\
1948 \\
734 \\
806 \\
2148 \\
515 \\
2352 \\
101 \\
2356 \\
2343 \\
511\end{array}$ \\
\hline 3 & $\begin{array}{l}114 \\
115 \\
116 \\
117 \\
118 \\
119 \\
120 \\
121 \\
122 \\
123 \\
124 \\
125 \\
126 \\
127\end{array}$ & $\begin{array}{l}3.015 \\
4.500 \\
5.079 \\
6.075 \\
3.021 \\
2.710 \\
2.164 \\
4.118 \\
6.576 \\
5.508 \\
3.517 \\
3.477 \\
5.890 \\
4.529\end{array}$ & $\begin{array}{r}12.23 \\
19.95 \\
25.13 \\
27.34 \\
18.88 \\
13.39 \\
9.40 \\
20.60 \\
25.81 \\
21.08 \\
15.62 \\
22.10 \\
28.95 \\
30.42\end{array}$ & $\begin{array}{r}1558 \\
1242 \\
2105 \\
1407 \\
1354 \\
1433 \\
1253 \\
1232 \\
1513 \\
653 \\
1131 \\
913 \\
1240 \\
1202\end{array}$ & $\begin{array}{l}.571 \\
.571 \\
.571 \\
.486 \\
.571 \\
.571 \\
.571 \\
.600 \\
.571 \\
.500 \\
.571 \\
.571 \\
.600 \\
.571\end{array}$ & $\begin{array}{r}1945 \\
229 \\
55 \\
1447 \\
2137 \\
740 \\
2158 \\
2201 \\
2201 \\
2400 \\
532 \\
1940 \\
2358 \\
610\end{array}$ \\
\hline
\end{tabular}


Table 3. Summary of 1984 data: Raft station wind speed--(1) daily average wind speed at 1,2 and 3 meters above lake surface, (2) dally maximum and minimum wind speed at the level and the time they occurred. (continued)

\begin{tabular}{|c|c|c|c|c|c|c|}
\hline $\begin{array}{l}\text { HEIGHT OF } \\
\text { MEASUREMENT } \\
\text { AEOUE LARE } \\
\text { SURFACE } \\
(m)\end{array}$ & $\underset{\text { JIAY I AN }}{\text { JUL }}$ & $\begin{array}{l}\text { IIAILY } \\
\text { AUERAGE } \\
\text { WINII } \\
\text { SFEEII } \\
(m i / h)\end{array}$ & $\begin{array}{l}\text { IIAILY } \\
\text { MAXIMUH } \\
\text { WINII } \\
\text { SFEEI } \\
(\pi, / h)\end{array}$ & $\begin{array}{l}\text { TIME OF } \\
\text { MAXIMUM } \\
\text { WINII } \\
\text { SFEEII } \\
\text { (h) }\end{array}$ & $\begin{array}{l}\text { LIAILY } \\
\text { MINIMUM } \\
\text { WINI } \\
\text { SFEEII } \\
(m I / n)\end{array}$ & $\begin{array}{l}\text { TIME OF } \\
\text { MINIMUM } \\
\text { WINII } \\
\text { SFEEII } \\
\text { (h) }\end{array}$ \\
\hline 1 & $\begin{array}{l}128 . \\
129 . \\
130 . \\
131 . \\
132 . \\
133 . \\
134 .\end{array}$ & $\begin{array}{l}1.896 \\
1.451 \\
3.026 \\
3.904 \\
2.615 \\
1.226 \\
2.206\end{array}$ & $\begin{array}{l}12.650 \\
14.010 \\
17.520 \\
22.980 \\
18.620 \\
18.280 \\
13.640\end{array}$ & $\begin{array}{l}1129 . \\
1059 . \\
1200 . \\
1451 . \\
1305 . \\
1718 . \\
1334 .\end{array}$ & $\begin{array}{r}.571 \\
.600 \\
.571 \\
.571 \\
.571 \\
.571 \\
.571\end{array}$ & $\begin{array}{l}2330 . \\
2400^{\circ} \\
18180^{\circ} \\
2210^{\circ} \\
2141 . \\
1922 . \\
2210^{\circ} .\end{array}$ \\
\hline 2 & $\begin{array}{l}128 . \\
129 . \\
130 . \\
131 . \\
132 . \\
133 . \\
134 .\end{array}$ & $\begin{array}{l}2.926 \\
2.185 \\
3.922 \\
4.904 \\
3.441 \\
2.057 \\
2.982\end{array}$ & $\begin{array}{l}15.880 \\
14.600 \\
18.030 \\
24.340 \\
17.940 \\
18.990 \\
13.270\end{array}$ & $\begin{array}{l}1524 . \\
1111 . \\
1239 . \\
1451 . \\
1305 . \\
1718 . \\
1259 .\end{array}$ & $\begin{array}{l}.571 \\
.600 \\
.600 \\
.571 \\
.232 \\
.571 \\
.600\end{array}$ & $\begin{array}{r}2330^{\circ} \\
23490^{\circ} \\
2328 . \\
2210^{\circ} \\
531 . \\
1332 . \\
2352 .\end{array}$ \\
\hline 3 & $\begin{array}{l}128 . \\
129 . \\
130 . \\
131 . \\
132 . \\
133 . \\
134 .\end{array}$ & $\begin{array}{l}2.715 \\
2.021 \\
4.002 \\
4.934 \\
3.407 \\
2.003 \\
2.977\end{array}$ & $\begin{array}{l}15.740 \\
15.080 \\
19.750 \\
22.980 \\
18.310 \\
18.480 \\
14.260\end{array}$ & $\begin{array}{l}1524 . \\
1111 . \\
1239 . \\
1451 . \\
1305 . \\
1718 . \\
1345 .\end{array}$ & $\begin{array}{l}.571 \\
.600 \\
.571 \\
.571 \\
.571 \\
.571 \\
.571\end{array}$ & $\begin{array}{l}2330 . \\
2356 . \\
1818 . \\
2210 . \\
2137 . \\
1311 . \\
2211 .\end{array}$ \\
\hline
\end{tabular}

\begin{tabular}{|c|c|c|c|c|c|c|}
\hline $\begin{array}{c}\text { HEIGHT OF } \\
\text { MEASUREMENT } \\
\text { ABOUE LAKE } \\
\text { SURFACE } \\
(m)\end{array}$ & $\begin{array}{l}\text { JUL I AN } \\
\text { IIAY }\end{array}$ & $\begin{array}{l}\text { IIAILY } \\
\text { AUERAGE } \\
\text { WINLI } \\
\text { SFEEI } \\
\left(m_{1} / n\right)\end{array}$ & $\begin{array}{l}\text { IIAILY } \\
\text { MAXIMUM } \\
\text { WINII } \\
\text { SFEEII } \\
(m 1 / h)\end{array}$ & $\begin{array}{l}\text { TIME OF } \\
\text { MAXIMUM } \\
\text { WINLI } \\
\text { SFEEII } \\
\text { (h) }\end{array}$ & $\begin{array}{l}\text { IIAILY } \\
\text { MINIMUM } \\
\text { WINII } \\
\text { SFEEII } \\
(m I / n)\end{array}$ & $\begin{array}{l}\text { TIME OF } \\
\text { MINIMUM } \\
\text { WINII } \\
\text { SFEEII } \\
\text { (n) }\end{array}$ \\
\hline 1 & $\begin{array}{l}135^{\circ} \\
1360^{\circ} \\
1370^{\circ} \\
1380^{\circ} \\
1390^{\circ} \\
140^{\circ} \\
141 .\end{array}$ & $\begin{array}{l}4.791 \\
4.053 \\
5.789 \\
2.873 \\
3.687 \\
2.283 \\
3.267\end{array}$ & $\begin{array}{l}21.650 \\
21.650 \\
23.120 \\
24.960 \\
22.750 \\
15.820 \\
14.830\end{array}$ & $\begin{array}{r}1635^{\circ} \\
1752^{\circ} \\
1146 . \\
1153 . \\
954 . \\
1407 . \\
730^{\circ} .\end{array}$ & $\begin{array}{l}.571 \\
.571 \\
.571 \\
.571 \\
.571 \\
.571 \\
.600\end{array}$ & $\begin{array}{r}1951 . \\
1955^{\circ} \\
2333 . \\
536 . \\
606 . \\
712 . \\
2400 .\end{array}$ \\
\hline 2 & $\begin{array}{l}135^{\circ} \\
1366^{\circ} \\
1370^{\circ} \\
138^{\circ} \\
139^{\circ} \\
140^{\circ} \\
141 .\end{array}$ & $\begin{array}{l}5.596 \\
4.805 \\
6.601 \\
4.073 \\
4.583 \\
3.001 \\
4.035\end{array}$ & $\begin{array}{l}23.490 \\
25.020 \\
23.600 \\
29.460 \\
23.770 \\
16.410 \\
17.010\end{array}$ & $\begin{array}{r}1837 . \\
1752 . \\
1458 . \\
1153 . \\
954 . \\
1402 . \\
730 .\end{array}$ & $\begin{array}{l}.571 \\
.571 \\
.571 \\
.571 \\
.600 \\
.571 \\
.571\end{array}$ & $\begin{array}{r}2058 . \\
656 . \\
2333 . \\
1146 . \\
2107 . \\
712 . \\
1924 .\end{array}$ \\
\hline 3 & $\begin{array}{l}135^{\circ} \\
1360^{\circ} \\
137 \circ^{\circ} \\
138^{\circ} \\
139^{\circ} \\
140^{\circ} \\
141 .\end{array}$ & $\begin{array}{l}5.731 \\
4.854 \\
6.771 \\
4.010 \\
4.611 \\
2.897 \\
4.115\end{array}$ & $\begin{array}{l}24.230 \\
25.330 \\
27.230 \\
29.630 \\
25.130 \\
17.580 \\
17.150\end{array}$ & $\begin{array}{r}1837 . \\
1752 . \\
1458 . \\
1153 . \\
954 . \\
1402 . \\
730 .\end{array}$ & $\begin{array}{l}.600 \\
.571 \\
.571 \\
.571 \\
.600 \\
.571 \\
.600\end{array}$ & $\begin{array}{r}2355 . \\
702 . \\
2333 . \\
1146 . \\
2118 . \\
720 . \\
2328 .\end{array}$ \\
\hline
\end{tabular}


Table 3. Summary of 1984 data: Raft station wind speed--(1) dally average wind speed at 1,2 and 3 meters above lake surface, (2) daily maximum and minimum wind speed at the level and the time they occurred. (continued)

\begin{tabular}{|c|c|c|c|c|c|c|}
\hline $\begin{array}{l}\text { HEIGHT OF } \\
\text { MEASUFEMENT } \\
\text { ABOUE LAKE } \\
\text { SUKFACE } \\
\left(m_{1}\right)\end{array}$ & $\underset{\text { IIAY }}{\text { JUL IAN }}$ & $\begin{array}{l}\text { DAILY } \\
\text { AUEFAGE } \\
\text { WINI } \\
\text { SFEEII } \\
(n 13 / n)\end{array}$ & $\begin{array}{l}\text { [IAILY } \\
\text { MAXIMUM } \\
\text { WINII } \\
\text { SFEEI } \\
(m 1 / n)\end{array}$ & $\begin{array}{l}\text { TIME OF } \\
\text { MAXIMUM } \\
\text { WINI } \\
\text { SFEEII } \\
\text { (h) }\end{array}$ & $\begin{array}{l}\text { IIAILY } \\
\text { MINIMUM } \\
\text { WINU } \\
\text { SFEEI } \\
(m i / h)\end{array}$ & $\begin{array}{l}\text { TIME OF } \\
\text { MINIMUM } \\
\text { WINII } \\
\text { SPEEI } \\
\text { (n) }\end{array}$ \\
\hline 1 & $\begin{array}{l}142 . \\
143 . \\
144 . \\
145 . \\
146 . \\
147 . \\
148 . \\
149 . \\
150 . \\
151 . \\
152 . \\
153 . \\
154 . \\
155 .\end{array}$ & $\begin{array}{l}3.469 \\
1.802 \\
3.526 \\
6.245 \\
2.431 \\
2.808 \\
4.272 \\
1.319 \\
2.434 \\
1.552 \\
5.364 \\
5.293 \\
5.005 \\
1.950\end{array}$ & $\begin{array}{r}15.140 \\
11.350 \\
23.770 \\
26.830 \\
18.590 \\
23.890 \\
17.180 \\
5.948 \\
16.190 \\
10.130 \\
19.020 \\
23.380 \\
27.820 \\
11.800\end{array}$ & $\begin{array}{r}957 . \\
1124 . \\
1547 . \\
1210 . \\
1413 . \\
1615 . \\
850 . \\
1310 . \\
747 . \\
2301 . \\
2159 . \\
545 . \\
946 . \\
1507 .\end{array}$ & $\begin{array}{l}.571 \\
.571 \\
.571 \\
.600 \\
.600 \\
.571 \\
.571 \\
.571 \\
.600 \\
.600 \\
.600 \\
.600 \\
.600 \\
.600\end{array}$ & $\begin{array}{r}2142 . \\
2217 . \\
647 . \\
2358 . \\
2359 . \\
722 . \\
145 . \\
2004 . \\
2359 . \\
2245 . \\
2336 . \\
2345 . \\
2359 . \\
2400 .\end{array}$ \\
\hline 2 & $\begin{array}{l}142 . \\
143 . \\
144 . \\
145 . \\
146 . \\
147 . \\
148 . \\
149 . \\
150 . \\
151 . \\
152 . \\
153 . \\
154 . \\
155 .\end{array}$ & $\begin{array}{l}4.329 \\
2.341 \\
3.968 \\
6.920 \\
3.229 \\
3.429 \\
4.855 \\
1.995 \\
2.961 \\
1.941 \\
6.042 \\
6.039 \\
5.386 \\
2.810\end{array}$ & $\begin{array}{r}15.880 \\
15.680 \\
26.120 \\
27.250 \\
18.480 \\
24.820 \\
18.850 \\
6.656 \\
16.330 \\
9.340 \\
19.670 \\
25.410 \\
29.860 \\
12.340\end{array}$ & $\begin{array}{r}1418, \\
1152 . \\
1247 . \\
1210^{\circ} \\
1413 . \\
1615 . \\
827 . \\
1310 . \\
720 . \\
2301 . \\
2109 . \\
545 . \\
946 . \\
1507 .\end{array}$ & $\begin{array}{l}.571 \\
.175 \\
.571 \\
.571 \\
.571 \\
.571 \\
.571 \\
.571 \\
.543 \\
.571 \\
.600 \\
.600 \\
.600 \\
.600\end{array}$ & $\begin{array}{r}2142 . \\
526 . \\
646 . \\
359 . \\
747 . \\
2031 . \\
141 . \\
2004 . \\
435 . \\
1441 . \\
1313 . \\
2345 . \\
2359 . \\
2257 .\end{array}$ \\
\hline 3 & $\begin{array}{l}142 . \\
143 . \\
144 . \\
145 . \\
146 . \\
147 . \\
148 . \\
149 . \\
150 . \\
151 . \\
152 . \\
153 . \\
154 . \\
155 .\end{array}$ & $\begin{array}{l}4.273 \\
2.258 \\
4.086 \\
7.055 \\
3.227 \\
3.356 \\
4.925 \\
1.073 \\
2.879 \\
1.760 \\
5.199 \\
5.811 \\
5.064 \\
2.675\end{array}$ & $\begin{array}{r}15.310 \\
15.740 \\
25.130 \\
29.970 \\
19.950 \\
23.180 \\
19.980 \\
0.769 \\
16.730 \\
8.070 \\
16.270 \\
19.640 \\
30.110 \\
12.510\end{array}$ & $\begin{array}{r}1028 . \\
1152 . \\
1247 . \\
1040 . \\
1502 . \\
1615 . \\
1237 . \\
1310 . \\
720 . \\
2301 . \\
2109 . \\
545 . \\
1030 . \\
1507 .\end{array}$ & $\begin{array}{l}.571 \\
.571 \\
.571 \\
.571 \\
.571 \\
.571 \\
.571 \\
.571 \\
.203 \\
.600 \\
.730 \\
.571 \\
.034 \\
.571\end{array}$ & $\begin{array}{r}2143 . \\
2217^{\circ} \\
648 . \\
400 . \\
2238 . \\
2031^{\circ} \\
1855^{\circ} \\
2004 . \\
1211^{\circ} \\
445^{\circ} \\
341 . \\
816 . \\
934 . \\
2104 .\end{array}$ \\
\hline
\end{tabular}


Table 3. Summary of 1984 data: Raft station wind speed--(1) daily average wind speed at 1,2 and 3 meters above lake surface, (2) daily maximum and minimum wind speed at the level and the time they occurred. (continued)

\begin{tabular}{|c|c|c|c|c|c|c|}
\hline $\begin{array}{l}\text { HEIGHT OF } \\
\text { MEASUREMENT } \\
\text { ABOUE LAKE } \\
\text { SURFACE } \\
\text { (m) }\end{array}$ & $\begin{array}{l}\text { JUL I AN } \\
\text { IIAY }\end{array}$ & $\begin{array}{l}\text { IIAILY } \\
\text { AUERAGE } \\
\text { WINII } \\
\text { SFEEII } \\
(m i \mathrm{~h} / \mathrm{h})\end{array}$ & $\begin{array}{l}\text { IIAILY } \\
\text { MAXIMUM } \\
\text { WINI } \\
\text { SFEED } \\
\left(m_{1}, h\right)\end{array}$ & $\begin{array}{l}\text { TIME OF } \\
\text { MAXIMUM } \\
\text { WINII } \\
\text { SFEED } \\
\text { ( } h)\end{array}$ & $\begin{array}{l}\text { IIAILY } \\
\text { MINIMUM } \\
\text { WINI } \\
\text { SPEED } \\
(n I 1 / h)\end{array}$ & $\begin{array}{l}\text { TIME OF } \\
\text { MINIMUM } \\
\text { WINII } \\
\text { SPEEI } \\
\text { (h) }\end{array}$ \\
\hline 1 & $\begin{array}{l}156 . \\
157 . \\
158 . \\
159 . \\
160 .\end{array}$ & $\begin{array}{r}2.976 \\
2.920 \\
.955 \\
.989 \\
2.080\end{array}$ & $\begin{array}{r}30.680 \\
18.480 \\
7.950 \\
7.533 \\
25.730\end{array}$ & $\begin{array}{l}1342 . \\
1215 . \\
1348 . \\
1405 . \\
1432 .\end{array}$ & $\begin{array}{l}.571 \\
.571 \\
.571 \\
.571 \\
.571\end{array}$ & $\begin{array}{r}1954 . \\
927 . \\
1321 . \\
2151 . \\
2240 .\end{array}$ \\
\hline 2 & $\begin{array}{l}156 . \\
157 . \\
158 . \\
159 . \\
160 .\end{array}$ & $\begin{array}{l}3.952 \\
3.755 \\
1.780 \\
1.752 \\
2.946\end{array}$ & $\begin{array}{r}33.230 \\
18.370 \\
7.788 \\
7.561 \\
29.490\end{array}$ & $\begin{array}{l}1342 . \\
1433 . \\
1348 . \\
1405 . \\
1432 .\end{array}$ & $\begin{array}{l}.571 \\
.571 \\
.600 \\
.571 \\
.571\end{array}$ & $\begin{array}{r}2206 . \\
928 . \\
2400 . \\
2151 . \\
857 .\end{array}$ \\
\hline 3 & $\begin{array}{l}156 . \\
157 . \\
158 . \\
159 . \\
160 .\end{array}$ & $\begin{array}{l}3.696 \\
3.625 \\
1.518 \\
1.591 \\
2.865\end{array}$ & $\begin{array}{r}34.980 \\
18.400 \\
6.486 \\
7.731 \\
23.410\end{array}$ & $\begin{array}{l}1342 . \\
1433 . \\
1348 . \\
1405 . \\
1432 .\end{array}$ & $\begin{array}{l}.571 \\
.571 \\
.317 \\
.571 \\
.571\end{array}$ & $\begin{array}{l}2206 . \\
2148 . \\
1204 . \\
2151 . \\
857 .\end{array}$ \\
\hline
\end{tabular}


Table 3. Summary of 1984 data: Raft station wind speed--(1) dafly average wind speed at 1,2 and 3 meters above lake surface, (2) datly maximum and minimum wind speed at the level and the time they occurred. (continued)

\begin{tabular}{|c|c|c|c|c|c|c|}
\hline $\begin{array}{c}\text { HEIGHT OF } \\
\text { MEASUFEMENT } \\
\text { ABOUE LAKE } \\
\text { SUKFACE } \\
(m)\end{array}$ & $\begin{array}{l}\text { JUL I AN } \\
\text { IIAY }\end{array}$ & $\begin{array}{l}\text { IIAILY } \\
\text { AUERAGE } \\
\text { WINII } \\
\text { SPEEII } \\
(m) / h)\end{array}$ & $\begin{array}{l}\text { IIAILY' } \\
\text { MAXIMUM } \\
\text { WINII } \\
\text { SFEEII } \\
(\mathrm{mi} / \mathrm{h})\end{array}$ & $\begin{array}{l}\text { TIME OF } \\
\text { MAXIMUM } \\
\text { WINI } \\
\text { SFEEI } \\
\text { (h) }\end{array}$ & $\begin{array}{l}\text { IIAILY } \\
\text { MINIMUM } \\
\text { WINII } \\
\text { SFEEII } \\
(\pi 1 / h)\end{array}$ & $\begin{array}{l}\text { TIME OF } \\
\text { MINIMUM } \\
\text { WINII } \\
\text { SFEEI } \\
\text { (h) }\end{array}$ \\
\hline 1 & $\begin{array}{l}161 . \\
162 . \\
163 . \\
164 . \\
165^{\circ} \\
1660^{\circ} \\
167 . \\
168 . \\
169 . \\
170 . \\
171 . \\
172 . \\
173 .\end{array}$ & $\begin{array}{l}3.150 \\
1.602 \\
4.811 \\
3.276 \\
3.388 \\
3.888 \\
7.051 \\
2.023 \\
2.383 \\
1.144 \\
3.350 \\
6.012 \\
4.846\end{array}$ & $\begin{array}{r}22.300 \\
12.370 \\
34.470 \\
18.740 \\
19.360 \\
26.830 \\
29.040 \\
16.300 \\
15.230 \\
6.344 \\
21.480 \\
20.890 \\
26.260\end{array}$ & $\begin{array}{r}1235 . \\
1300^{\circ} \\
1804{ }^{\circ} \\
136{ }^{\circ} \\
1527^{\circ} \\
2358 . \\
645^{\circ} \\
1044 . \\
1547^{\circ} \\
2124 . \\
2103 . \\
1316 . \\
2001 .\end{array}$ & $\begin{array}{r}.571 \\
.571 \\
.571 \\
.571 \\
.600 \\
.571 \\
.571 \\
.571 \\
.430 \\
.600 \\
.571 \\
.571 \\
.571\end{array}$ & $\begin{array}{r}6277^{\circ} \\
1840^{\circ} \\
1245^{\circ} \\
1435{ }^{\circ} \\
2358 . \\
1227^{\circ} \\
2344 . \\
412 . \\
1229 . \\
2359 . \\
1017 . \\
726 . \\
1614 .\end{array}$ \\
\hline 2 & $\begin{array}{l}161 . \\
162 . \\
163 . \\
164 . \\
165 . \\
166 . \\
167 . \\
168 . \\
169 . \\
170 . \\
171 . \\
172 . \\
173 .\end{array}$ & $\begin{array}{l}4.115 \\
2.541 \\
5.622 \\
3.897 \\
3.892 \\
4.418 \\
7.802 \\
2.617 \\
3.053 \\
1.672 \\
3.810 \\
6.636 \\
5.455\end{array}$ & $\begin{array}{l}23.630 \\
13.160 \\
27.370 \\
18.080 \\
19.080 \\
30.250 \\
30.590 \\
16.920 \\
15.450 \\
6.684 \\
22.640 \\
21.280 \\
26.800\end{array}$ & $\begin{array}{r}1235 . \\
1300 . \\
1804 . \\
136{ }^{\circ} \\
1527 . \\
2059 . \\
319 . \\
1044 . \\
1547 . \\
2124 . \\
2103 . \\
1316 . \\
2001 .\end{array}$ & $\begin{array}{l}.571 \\
.571 \\
.571 \\
.571 \\
.600 \\
.571 \\
.571 \\
.571 \\
.571 \\
.373 \\
.571 \\
.600 \\
.600\end{array}$ & $\begin{array}{r}627 . \\
2309^{\circ} \\
820^{\circ} \\
2103 . \\
2356 . \\
1457 . \\
23430^{\circ} \\
836 . \\
21390^{\circ} \\
922 . \\
743 . \\
2346 . \\
2400 .\end{array}$ \\
\hline 3 & $\begin{array}{l}1610^{\circ} \\
162{ }^{\circ} \\
163{ }^{\circ} \\
164{ }^{\circ} \\
165^{\circ} \\
166{ }^{\circ} \\
167{ }^{\circ} \\
168{ }^{\circ} \\
169{ }^{\circ} \\
170 . \\
171 . \\
172 . \\
173 .\end{array}$ & $\begin{array}{l}4.156 \\
2.410 \\
5.687 \\
3.898 \\
3.905 \\
4.482 \\
8.020 \\
2.585 \\
3.102 \\
1.779 \\
3.965 \\
6.809 \\
5.552\end{array}$ & $\begin{array}{r}22.870 \\
13.390 \\
29.120 \\
17.910 \\
19.100 \\
32.230 \\
32.230 \\
16.470 \\
15.910 \\
6.995 \\
22.900 \\
21.480 \\
27.230\end{array}$ & $\begin{array}{c}12355^{\circ} \\
1300 . \\
1804{ }^{\circ} \\
1446 . \\
15280^{\circ} \\
2059 . \\
319 . \\
1044 . \\
1547 . \\
2009 . \\
2103 . \\
1316 . \\
2001 .\end{array}$ & $\begin{array}{r}.571 \\
.571 \\
.571 \\
.571 \\
.600 \\
.571 \\
.571 \\
.571 \\
.571 \\
.571 \\
.571 \\
.600 \\
.600\end{array}$ & 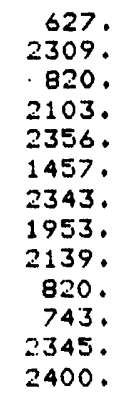 \\
\hline
\end{tabular}


Table 3. Summary of 1984 data: Raft station wind speed--(1) daily average wind speed at 1,2 and 3 meters above lake surface, (2) daily maximum and minimum wind speed at the level and the time they occurred. (continued)

\begin{tabular}{|c|c|c|c|c|c|c|}
\hline $\begin{array}{c}\text { HEIGHT OF } \\
\text { MEASUREMENT } \\
\text { ABOUE LAKE } \\
\text { SUFFACE } \\
\text { (mi) }\end{array}$ & $\underset{\text { JUAY IAN }}{\text { JUL }}$ & $\begin{array}{l}\text { IIAILY } \\
\text { AUERAGE } \\
\text { WINII } \\
\text { SFEEI } \\
(\mathrm{mi} / \mathrm{h})\end{array}$ & $\begin{array}{l}\text { UAILY } \\
\text { MAXIMUM } \\
\text { WINII } \\
\text { SFEEII } \\
(m i / h)\end{array}$ & $\begin{array}{l}\text { TIME OF } \\
\text { MAXIMUM } \\
\text { WINI } \\
\text { SFEEII } \\
\text { (h) }\end{array}$ & $\begin{array}{l}\text { IIAILY } \\
\text { MINIMUM } \\
\text { WINII } \\
\text { SFEEII } \\
(m i / h)\end{array}$ & $\begin{array}{l}\text { TIME OF } \\
\text { MINIMUM } \\
\text { WINII } \\
\text { SFEEI } \\
\text { (h) }\end{array}$ \\
\hline 1 & $\begin{array}{l}174 . \\
175 . \\
176 . \\
177 . \\
178 .\end{array}$ & $\begin{array}{l}4.236 \\
2.940 \\
3.626 \\
1.797 \\
3.382\end{array}$ & $\begin{array}{l}24.250 \\
16.560 \\
14.150 \\
10.640 \\
20.600\end{array}$ & $\begin{array}{l}1100 . \\
1102 . \\
1031 . \\
1820 . \\
1004 .\end{array}$ & $\begin{array}{r}.571 \\
.571 \\
.571 \\
.600 \\
.571\end{array}$ & $\begin{array}{r}226 . \\
852 . \\
644 . \\
2350 . \\
2235 .\end{array}$ \\
\hline 2 & $\begin{array}{l}174 . \\
175 \\
176^{\circ} \\
177 \\
178 .\end{array}$ & $\begin{array}{l}4.787 \\
3.523 \\
4.245 \\
2.453 \\
4.108\end{array}$ & $\begin{array}{l}23.520 \\
17.580 \\
15.960 \\
11.430 \\
21.080\end{array}$ & $\begin{array}{l}1100 \\
1102 \\
1150 \\
1820 \\
1004\end{array}$ & $\begin{array}{l}.571 \\
.571 \\
.600 \\
.600 \\
.571\end{array}$ & $\begin{array}{r}730 . \\
852 . \\
1931 . \\
2338 . \\
557 .\end{array}$ \\
\hline 3 & $\begin{array}{l}174 . \\
175 \\
176 \\
177 \\
178\end{array}$ & $\begin{array}{l}4.902 \\
3.601 \\
4.369 \\
2.505 \\
4.203\end{array}$ & $\begin{array}{l}23.910 \\
17.890 \\
16.390 \\
11.180 \\
22.190\end{array}$ & $\begin{array}{l}1123 . \\
1102 . \\
1150 . \\
1025 . \\
1004 .\end{array}$ & $\begin{array}{l}.571 \\
.571 \\
.600 \\
.600 \\
.571\end{array}$ & $\begin{array}{r}730 . \\
852 . \\
1956 . \\
2324 . \\
554 .\end{array}$ \\
\hline
\end{tabular}

\begin{tabular}{|c|c|c|c|c|c|c|}
\hline $\begin{array}{l}\text { HEIGHT OF } \\
\text { MEASUREMENT } \\
\text { ABOUE LAKE } \\
\text { SUFFACE } \\
(m)\end{array}$ & $\begin{array}{l}\text { JUL. IAN } \\
\text { IIAY }\end{array}$ & $\begin{array}{l}\text { IIAILY } \\
\text { AUERAGE } \\
\text { WINI } \\
\text { SFEEI } \\
(m 1 / n)\end{array}$ & $\begin{array}{l}\text { IIAILY } \\
\text { MAXIMUM } \\
\text { WINII } \\
\text { SFEEII } \\
(n i / h)\end{array}$ & $\begin{array}{l}\text { TIME OF } \\
\text { MAXIMUM } \\
\text { WINII } \\
\text { SFEEI } \\
\text { (h) }\end{array}$ & $\begin{array}{l}\text { IIAILY } \\
\text { MINIMUM } \\
\text { WINII } \\
\text { SFEEI } \\
(m i / h)\end{array}$ & $\begin{array}{l}\text { TIME OF } \\
\text { MINIMUM } \\
\text { WINI } \\
\text { SFEEI } \\
\text { (h) }\end{array}$ \\
\hline.----1 & ---- & $----\cdots$ & $---\cdots--$ & ----- & ----- & ---- \\
\hline 1 & $\begin{array}{l}179 . \\
180 . \\
181 . \\
182^{\circ} \\
183 .\end{array}$ & $\begin{array}{r}1.640 \\
2.487 \\
1.817 \\
.921 \\
1.951\end{array}$ & $\begin{array}{r}10.810 \\
18.230 \\
15.510 \\
4.675 \\
9.400\end{array}$ & $\begin{array}{r}1414 . \\
1149 . \\
1439 . \\
905^{\circ} \\
1645^{\circ} .\end{array}$ & $\begin{array}{l}.571 \\
.600 \\
.571 \\
.571 \\
.571\end{array}$ & $\begin{array}{l}2306 . \\
2400 . \\
2231 . \\
2213 . \\
805 .\end{array}$ \\
\hline 2 & $\begin{array}{l}179 . \\
180 . \\
181 . \\
182 . \\
183 .\end{array}$ & $\begin{array}{l}2.482 \\
3.074 \\
2.395 \\
1.598 \\
2.582\end{array}$ & $\begin{array}{r}13.920 \\
18.340 \\
17.740 \\
5.382 \\
10.470\end{array}$ & $\begin{array}{l}1414 . \\
1149 . \\
1435 . \\
2111 . \\
1645 .\end{array}$ & $\begin{array}{l}.571 \\
.571 \\
.571 \\
.571 \\
.571\end{array}$ & $\begin{array}{l}2306 . \\
1751 . \\
2229 . \\
1727 . \\
2137 .\end{array}$ \\
\hline 3 & $\begin{array}{l}179 . \\
180^{\circ} \\
181 . \\
182 . \\
183 .\end{array}$ & $\begin{array}{l}2.495 \\
3.139 \\
2.385 \\
1.520 \\
2.556\end{array}$ & $\begin{array}{r}14.830 \\
16.500 \\
18.000 \\
5.411 \\
10.560\end{array}$ & $\begin{array}{l}1414 . \\
1149 . \\
1439 . \\
2110^{\circ} . \\
1645^{\circ} .\end{array}$ & $\begin{array}{l}.571 \\
.600 \\
.571 \\
.571 \\
.571\end{array}$ & $\begin{array}{l}2306 . \\
2358 . \\
2229 . \\
1727 . \\
2137 .\end{array}$ \\
\hline
\end{tabular}


Table 3. Summary of 1984 data: Raft station wind speed--(1) dally average wind speed at 1,2 and 3 meters above lake surface, (2) daily maximum and minimum wind speed at the level and the time they occurred. (continued)

\begin{tabular}{|c|c|c|c|c|c|c|}
\hline $\begin{array}{l}\text { HEIGHT OF } \\
\text { MEASUREMENT } \\
\text { AHOUE LAFE } \\
\text { SUFFACE } \\
\text { (m) }\end{array}$ & $\underset{\text { JIAY I AN }}{\text { JUL_ }}$ & $\begin{array}{l}\text { IIAILY } \\
\text { AVEFAGE } \\
\text { WINII } \\
\text { SFEEII } \\
(m 1 / h)\end{array}$ & $\begin{array}{l}\text { IIAILY } \\
\text { MAXIMUM } \\
\text { WINII } \\
\text { SFEEI } \\
(m 1 / n)\end{array}$ & $\begin{array}{l}\text { TIME OF } \\
\text { MAXIMUM } \\
\text { WINEI } \\
\text { SPEEII } \\
\text { (h) }\end{array}$ & $\begin{array}{l}\text { UAILY } \\
\text { MINIMUM } \\
\text { WINII } \\
\text { SFEE I } \\
(m 2 / n)\end{array}$ & $\begin{array}{l}\text { TIME OF } \\
\text { MINIMUM } \\
\text { WINI } \\
\text { SFEEI } \\
\text { (h) }\end{array}$ \\
\hline 1 & $\begin{array}{l}184 . \\
185^{\circ} \\
186^{\circ} \\
187 . \\
180^{\circ} \\
189 . \\
190 .\end{array}$ & $\begin{array}{l}2.250 \\
2.209 \\
1.720 \\
1.310 \\
2.799 \\
2.318 \\
5.181\end{array}$ & $\begin{array}{r}13.750 \\
23.040 \\
10.080 \\
7.788 \\
13.610 \\
15.710 \\
20.860\end{array}$ & $\begin{array}{r}1018 . \\
1425 . \\
1334 . \\
913 . \\
1205 . \\
50 \\
1258 .\end{array}$ & $\begin{array}{r}.600 \\
.571 \\
.571 \\
.600 \\
.600 \\
.571 \\
.571\end{array}$ & $\begin{array}{l}2355 . \\
1650 . \\
1453 . \\
2400 . \\
2328 . \\
1657 . \\
2347 .\end{array}$ \\
\hline 2 & $\begin{array}{l}184 . \\
185^{\circ} \\
186^{\circ} \\
187 . \\
188^{\circ} \\
189 . \\
190^{\circ} .\end{array}$ & $\begin{array}{l}3.002 \\
2.806 \\
2.333 \\
1.859 \\
3.280 \\
2.812 \\
5.762\end{array}$ & $\begin{array}{r}14.350 \\
24.250 \\
10.700 \\
8.970 \\
13.160 \\
15.620 \\
23.660\end{array}$ & $\begin{array}{r}1018 . \\
1425 . \\
1334 . \\
913 . \\
1205 . \\
50 . \\
1258 .\end{array}$ & $\begin{array}{l}.600 \\
.600 \\
.571 \\
.571 \\
.600 \\
.600 \\
.600\end{array}$ & $\begin{array}{l}2340 . \\
2400 . \\
2028 . \\
1951 . \\
2318 . \\
2217 . \\
2357 .\end{array}$ \\
\hline 3 & $\begin{array}{l}184 . \\
185 . \\
186 . \\
187 . \\
188 . \\
189 . \\
190 .\end{array}$ & $\begin{array}{l}3.030 \\
2.806 \\
2.303 \\
1.804 \\
3.358 \\
2.854 \\
5.937\end{array}$ & $\begin{array}{r}14.000 \\
24.170 \\
11.720 \\
4.280 \\
14.260 \\
15.5770 \\
24.080\end{array}$ & $\begin{array}{r}1018 . \\
1425 . \\
1302 . \\
913 . \\
1213 . \\
50 . \\
1258 .\end{array}$ & $\begin{array}{l}.600 \\
.571 \\
.600 \\
.571 \\
.600 \\
.571 \\
.600\end{array}$ & $\begin{array}{r}2347 . \\
758 . \\
2351 . \\
1951 . \\
2330 . \\
1657 . \\
2358 .\end{array}$ \\
\hline
\end{tabular}

\begin{tabular}{|c|c|c|c|c|c|c|}
\hline $\begin{array}{l}\text { HEIGHT OF } \\
\text { MEASUREMENT } \\
\text { AEOUE LAKE } \\
\text { SUFF ACE } \\
(m)\end{array}$ & $\begin{array}{l}\text { JULI IAN } \\
\text { IIAY }\end{array}$ & $\begin{array}{l}\text { IIAILY } \\
\text { AUEFIAGE } \\
\text { WINII } \\
\text { SF'EEII } \\
(m i / h)\end{array}$ & $\begin{array}{l}\text { IIAILY } \\
\text { MAXIMUM } \\
\text { WINI! } \\
\text { SFEE! } \\
(m i / h)\end{array}$ & $\begin{array}{l}\text { TIME OF } \\
\text { MAXIMUM } \\
\text { WINII } \\
\text { SFEE[I } \\
\text { (h) }\end{array}$ & $\begin{array}{l}\text { IIAILY } \\
\text { MINIMUM } \\
\text { WINII } \\
\text { SPEEII } \\
\left(m_{1} / / h\right)\end{array}$ & $\begin{array}{l}\text { TIME OF } \\
\text { MINIMUM } \\
\text { WINI } \\
\text { SPEEII } \\
\text { (h) }\end{array}$ \\
\hline 1 & $\begin{array}{l}191 . \\
192 . \\
193 . \\
194 . \\
195 . \\
196 .\end{array}$ & $\begin{array}{l}1.946 \\
1.816 \\
1.681\end{array}$ & $\begin{array}{r}9.060 \\
11.070 \\
9.850\end{array}$ & $\begin{array}{l}904 . \\
1131 . \\
1003 .\end{array}$ & $\begin{array}{l}.571 \\
.317 \\
.600\end{array}$ & $\begin{array}{l}2226 . \\
2043 . \\
235 .\end{array}$ \\
\hline 2 & $\begin{array}{l}191 . \\
192 . \\
193 . \\
194 . \\
195 . \\
196 .\end{array}$ & $\begin{array}{l}2.557 \\
2.446 \\
2.152\end{array}$ & $\begin{array}{r}8.920 \\
11.430 \\
9.680\end{array}$ & $\begin{array}{l}904 . \\
1131 . \\
1003 .\end{array}$ & $\begin{array}{r}.571 \\
.571 \\
.600\end{array}$ & $\begin{array}{r}2226 . \\
944 . \\
2353 .\end{array}$ \\
\hline 3 & $\begin{array}{l}191 . \\
192 . \\
193 . \\
194 . \\
195 . \\
196 .\end{array}$ & $\begin{array}{l}2.570 \\
2.425 \\
2.105\end{array}$ & $\begin{array}{r}9.340 \\
11.920 \\
10.500\end{array}$ & $\begin{array}{l}904 . \\
1131 . \\
1003 .\end{array}$ & $\begin{array}{r}.571 \\
.571 \\
.571\end{array}$ & $\begin{array}{c}2226 . \\
1143 . \\
834 .\end{array}$ \\
\hline
\end{tabular}


Table 3. Summary of 1984 data: Raft station wind speed--(1) daily average wind speed at 1,2 and 3 meters above lake surface, (2) daily maximum and minimum wind speed at the level and the time they occurred. (continued)

\begin{tabular}{|c|c|c|c|c|c|c|}
\hline $\begin{array}{l}\text { HEIGHT OF } \\
\text { MEASUFEMENT } \\
\text { AEOUE LAKE } \\
\text { SUFFACE } \\
(m i)\end{array}$ & $\begin{array}{l}\text { IUL. IAN } \\
\text { IIAY }\end{array}$ & $\begin{array}{l}\text { IIAILY } \\
\text { AUEFIAGE } \\
\text { WINI } \\
\text { SFEEII } \\
\left(n_{2} / h\right)\end{array}$ & $\begin{array}{l}\text { IIAILY } \\
\text { MAXIMUM } \\
\text { WINII } \\
\text { SFEEII } \\
(n 1 / h)\end{array}$ & $\begin{array}{l}\text { TIME OF } \\
\text { MAXIMUM } \\
\text { WINII } \\
\text { SPEEII } \\
\text { (n) }\end{array}$ & $\begin{array}{l}\text { IIAILY } \\
\text { MINIMUM } \\
\text { WINI } \\
\text { SFEEI } \\
(m I / h)\end{array}$ & $\begin{array}{l}\text { TIME OF } \\
\text { MINIMUM } \\
\text { WINII } \\
\text { SFEEII } \\
\text { (h) }\end{array}$ \\
\hline$---------m$ & $-\infty-1$ & ----- & ------ & $---m--$ & $----m$ & ----- \\
\hline 1 & $\begin{array}{l}197 . \\
198 . \\
199 . \\
200 . \\
201 . \\
202 . \\
203 .\end{array}$ & $\begin{array}{l}2.568 \\
1.764 \\
2.442 \\
2.502 \\
1.780\end{array}$ & $\begin{array}{r}12.590 \\
7.901 \\
16.470 \\
15.170 \\
10.750\end{array}$ & $\begin{array}{l}1152 . \\
2101 . \\
1400 \\
1342 . \\
1603 .\end{array}$ & $\begin{array}{l}.571 \\
.571 \\
.571 \\
.571 \\
.571\end{array}$ & $\begin{array}{r}452 \\
2056 \\
2139 \\
1729 \\
2320\end{array}$ \\
\hline \multirow[t]{2}{*}{2} & $\begin{array}{l}197 . \\
198 .\end{array}$ & & & & & - \\
\hline & $\begin{array}{l}199 . \\
200 \\
201 \\
202 \\
203\end{array}$ & $\begin{array}{l}3.111 \\
2.230 \\
2.927 \\
2.967 \\
2.201\end{array}$ & $\begin{array}{r}12.570 \\
7.222 \\
15.760 \\
18.080 \\
10.840\end{array}$ & $\begin{array}{l}1307 \\
842 \\
1359 \\
1342 \\
1603\end{array}$ & $\begin{array}{l}.571 \\
.571 \\
.571 \\
.500 \\
.571\end{array}$ & $\begin{array}{l}1139 . \\
2056 . \\
2139 . \\
2357 . \\
326 .\end{array}$ \\
\hline \multirow[t]{2}{*}{3} & $\begin{array}{l}197 . \\
198 .\end{array}$ & & & & & \\
\hline & $\begin{array}{l}199 . \\
200 . \\
201 . \\
202 . \\
203 .\end{array}$ & $\begin{array}{l}3.127 \\
2.204 \\
2.934 \\
2.940 \\
2.143\end{array}$ & $\begin{array}{r}12.710 \\
6.712 \\
16.250 \\
17.070 \\
10.640\end{array}$ & $\begin{array}{l}1307 . \\
842 . \\
1359 . \\
1241 . \\
1603 .\end{array}$ & $\begin{array}{l}.571 \\
.571 \\
.571 \\
.600 \\
.571\end{array}$ & $\begin{array}{l}1139 \\
2056 \\
2138 \\
2400 \\
2320\end{array}$ \\
\hline
\end{tabular}

\begin{tabular}{|c|c|c|c|c|c|c|}
\hline $\begin{array}{l}\text { HEIGHT OF } \\
\text { MEASUFEMENT } \\
\text { AEOUE LAKE } \\
\text { SUFFACE. } \\
(m)\end{array}$ & $\begin{array}{l}\text { JUL IAN } \\
\text { IIAY }\end{array}$ & $\begin{array}{l}\text { IIAILY } \\
\text { AUERAGE } \\
\text { WINI } \\
\text { SFEEII } \\
(m I / h)\end{array}$ & $\begin{array}{l}\text { LIAILY } \\
\text { MAXIMUM } \\
\text { WINII } \\
\text { SF'EEII } \\
(m 1 / n)\end{array}$ & $\begin{array}{l}\text { TIME OF } \\
\text { MAXIMUM } \\
\text { WINII } \\
\text { SFEEI } \\
\text { (h) }\end{array}$ & $\begin{array}{l}\text { IIAILY } \\
\text { MINIMUM } \\
\text { WINII } \\
\text { SPEEI } \\
(m i / n)\end{array}$ & $\begin{array}{l}\text { TIME OF } \\
\text { MINIMUM } \\
\text { WINII } \\
\text { SFEEII } \\
\text { (h) }\end{array}$ \\
\hline 1 & $\begin{array}{l}204 . \\
205 . \\
206 . \\
207 . \\
208 . \\
209 . \\
210 .\end{array}$ & $\begin{array}{l}2.136 \\
2.382 \\
6.478 \\
5.708 \\
1.760 \\
1.673 \\
2.777\end{array}$ & $\begin{array}{r}8.150 \\
12.910 \\
27.620 \\
22.560 \\
19.900 \\
13.080 \\
15.030\end{array}$ & $\begin{array}{r}1920 . \\
1309 . \\
1221 . \\
620 . \\
1050 . \\
1105 . \\
1226 .\end{array}$ & $\begin{array}{r}.571 \\
.571 \\
.571 \\
.571 \\
.571 \\
.571 \\
.740\end{array}$ & $\begin{array}{l}1224 . \\
2354 . \\
2317 . \\
2247 . \\
2112 . \\
1959 . \\
1049 .\end{array}$ \\
\hline 2 & $\begin{array}{l}204 . \\
205 . \\
206 . \\
207 . \\
208 . \\
209 . \\
210 .\end{array}$ & $\begin{array}{l}2.550 \\
2.843 \\
7.067 \\
6.290 \\
2.368 \\
2.337 \\
3.371\end{array}$ & $\begin{array}{r}9.110 \\
12.420 \\
34.780 \\
21.740 \\
19.580 \\
9.960 \\
16.670\end{array}$ & $\begin{array}{l}1450 . \\
1309 . \\
1221 . \\
620 . \\
1050 . \\
1105 . \\
1225 .\end{array}$ & $\begin{array}{l}.571 \\
.571 \\
.600 \\
.571 \\
.571 \\
.571 \\
.600\end{array}$ & $\begin{array}{l}2125 . \\
2354 . \\
2352 . \\
2247 . \\
2111^{\circ} \\
1959 . \\
2400 .\end{array}$ \\
\hline 3 & $\begin{array}{l}204 . \\
205 . \\
206 . \\
207 . \\
208 . \\
209 . \\
210 .\end{array}$ & $\begin{array}{l}\because .527 \\
\because .833 \\
7.284 \\
6.437 \\
2.373 \\
2.343 \\
3.426\end{array}$ & $\begin{array}{r}8.770 \\
13.270 \\
34.190 \\
23.910 \\
19.050 \\
9.310 \\
17.800\end{array}$ & $\begin{array}{r}1450 . \\
1309 . \\
1221 . \\
902 . \\
1050 . \\
1104 . \\
1225 .\end{array}$ & $\begin{array}{l}.571 \\
.571 \\
.600 \\
.571 \\
.571 \\
.571 \\
.571\end{array}$ & $\begin{array}{l}2125 . \\
2354 . \\
2351 . \\
2247 . \\
2111 . \\
712 . \\
2343 .\end{array}$ \\
\hline
\end{tabular}


Table 3. Summary of 1984 data: Raft station wind speed--(1) daily average wind speed at 1,2 and 3 meters above lake surface, (2) daily maximum and minimum wind speed at the level and the time they occurred. (continued)

\begin{tabular}{|c|c|c|c|c|c|c|}
\hline $\begin{array}{l}\text { HEIGHT OF } \\
\text { MEASUREMENT } \\
\text { ABOUE LAKE } \\
\text { SURFACE } \\
\text { (m) }\end{array}$ & $\underset{\text { JIAY }}{\text { JUL IAN }}$ & $\begin{array}{l}\text { IIAILY } \\
\text { AUERAGE } \\
\text { WINII } \\
\text { SFEEII } \\
(m, / h)\end{array}$ & $\begin{array}{l}\text { IIAILY } \\
\text { MAXIMUM } \\
\text { WINII } \\
\text { SFEEI } \\
\text { (mi/h) }\end{array}$ & $\begin{array}{l}\text { TIME OF } \\
\text { MAXIMUM } \\
\text { WINII } \\
\text { SFEEII } \\
\text { (h) }\end{array}$ & $\begin{array}{l}\text { IIAILY } \\
\text { MINIMUM } \\
\text { WINI } \\
\text { SFEEII } \\
(m I / h)\end{array}$ & $\begin{array}{l}\text { TIME OF } \\
\text { MINIMUM } \\
\text { WINII } \\
\text { SPEEII } \\
\text { (h) }\end{array}$ \\
\hline$--m--m--m--m$ & $--m-\infty$ & $-m---$ & $--m----$ & $---\infty-\cdots$ & $--\infty-\infty$ & ------ \\
\hline 1 & $\begin{array}{l}211 . \\
212 . \\
213 . \\
214 . \\
215 . \\
216 . \\
217 . \\
218 .\end{array}$ & $\begin{array}{l}2.107 \\
1.531 \\
1.799 \\
1.765 \\
2.349 \\
1.961 \\
2.623 \\
2.077\end{array}$ & $\begin{array}{r}9.990 \\
7.137 \\
12.140 \\
10.530 \\
11.380 \\
14.090 \\
16.130 \\
11.800\end{array}$ & $\begin{array}{r}1039^{\circ} \\
1503 . \\
1141^{\circ} \\
1402^{\circ} \\
358^{\circ} \\
1149^{\circ} \\
1153^{\circ} \\
1256 .\end{array}$ & $\begin{array}{l}.600 \\
.571 \\
.600 \\
.600 \\
.600 \\
.571 \\
.571 \\
.571\end{array}$ & $\begin{array}{r}2358 . \\
2248 . \\
2359 . \\
2400 . \\
2400 . \\
2024 . \\
17 . \\
17 .\end{array}$ \\
\hline 2 & $\begin{array}{l}211 . \\
212 . \\
213 . \\
214 . \\
215 . \\
216 . \\
217 . \\
218 .\end{array}$ & $\begin{array}{l}2.667 \\
2.122 \\
2.472 \\
2.620 \\
3.034 \\
2.623 \\
3.175 \\
2.689\end{array}$ & $\begin{array}{r}10.730 \\
6.967 \\
12.760 \\
11.830 \\
10.840 \\
13.420 \\
15.710 \\
12.170\end{array}$ & $\begin{array}{l}1203 . \\
1503 . \\
1141 . \\
2005 . \\
1012 . \\
1149 . \\
1153 . \\
1344 .\end{array}$ & $\begin{array}{l}.600 \\
.571 \\
.600 \\
.571 \\
.571 \\
.600 \\
.571 \\
.571\end{array}$ & $\begin{array}{r}2348 . \\
2019 . \\
2348 . \\
849 . \\
1300 . \\
2250 . \\
751 . \\
812 .\end{array}$ \\
\hline 3 & $\begin{array}{l}211 . \\
212 . \\
213 . \\
214 . \\
215 . \\
210 . \\
217 . \\
218 .\end{array}$ & $\begin{array}{l}2.643 \\
2.055 \\
2.413 \\
2.572 \\
3.011 \\
2.5599 \\
3.167 \\
2.659\end{array}$ & $\begin{array}{r}10.590 \\
7.080 \\
14.550 \\
10.560 \\
11.070 \\
13.900 \\
15.480 \\
12.140\end{array}$ & $\begin{array}{r}1039 . \\
1304 . \\
1139 . \\
134 \% . \\
859 . \\
1149 . \\
1153 . \\
1344 .\end{array}$ & $\begin{array}{l}.600 \\
.571 \\
.571 \\
.571 \\
.571 \\
.600 \\
.571 \\
.571\end{array}$ & $\begin{array}{r}2358 . \\
2019 . \\
942 . \\
849 . \\
1259 . \\
2353 . \\
15 . \\
812 .\end{array}$ \\
\hline
\end{tabular}

\begin{tabular}{|c|c|c|c|c|c|c|}
\hline $\begin{array}{l}\text { HEIGHT OF } \\
\text { MEASUREMENT } \\
\text { AEOUE LAKE } \\
\text { SURFACE } \\
\text { (m) }\end{array}$ & $\begin{array}{l}\text { JUL I AN } \\
\text { IIAY }\end{array}$ & $\begin{array}{l}\text { IIAILY } \\
\text { AUEFAGE } \\
\text { WINII } \\
\text { SFEEII } \\
(m i / h)\end{array}$ & $\begin{array}{l}\text { IIAILY } \\
\text { MAXIMUM } \\
\text { WINII } \\
\text { SFEEII } \\
(m i / n)\end{array}$ & $\begin{array}{l}\text { TIME OF } \\
\text { MAXIMUM } \\
\text { WINII } \\
\text { SFEEII } \\
\text { (h) }\end{array}$ & $\begin{array}{l}\text { IIAILY } \\
\text { MINIMUH } \\
\text { WINII } \\
\text { SFEEII } \\
(m i / h)\end{array}$ & $\begin{array}{l}\text { TIME OF } \\
\text { MINIMUM } \\
\text { WINC } \\
\text { SFEEII } \\
\text { (h) }\end{array}$ \\
\hline 1 & $\begin{array}{l}219 . \\
220 . \\
221 . \\
222 . \\
223 . \\
224 . \\
225 . \\
226 .\end{array}$ & $\begin{array}{r}1.812 \\
2.130 \\
1.856 \\
2.552 \\
1.840 \\
.763 \\
1.572 \\
1.145\end{array}$ & $\begin{array}{r}10.590 \\
10.360 \\
10.420 \\
11.970 \\
8.260 \\
4.137 \\
10.100 \\
6.061\end{array}$ & $\begin{array}{l}1332 . \\
1310 . \\
1519 . \\
1014 . \\
1402 . \\
1428 . \\
1307 . \\
1703 .\end{array}$ & $\begin{array}{l}.571 \\
.571 \\
.571 \\
.500 \\
.571 \\
.571 \\
.571 \\
.571\end{array}$ & $\begin{array}{r}717 . \\
1614 . \\
648 . \\
2400 . \\
2134 . \\
1004 . \\
1149 . \\
1227 .\end{array}$ \\
\hline 2 & $\begin{array}{l}219 . \\
220 . \\
221 . \\
222 . \\
223 . \\
224 . \\
225 . \\
226 .\end{array}$ & $\begin{array}{l}2.395 \\
2.750 \\
2.539 \\
3.204 \\
2.588 \\
1.352 \\
2.548 \\
1.645\end{array}$ & $\begin{array}{r}13.100 \\
11.010 \\
10.640 \\
11.770 \\
9.060 \\
4.335 \\
12.140 \\
6.260\end{array}$ & $\begin{array}{l}1332 . \\
1435 . \\
1519 . \\
1613 . \\
1402 . \\
1428 . \\
1202 . \\
1703 .\end{array}$ & $\begin{array}{l}.571 \\
.600 \\
.571 \\
.600 \\
.571 \\
.571 \\
.571 \\
.571\end{array}$ & $\begin{array}{r}717 . \\
2358 . \\
650 . \\
2359 . \\
2134 . \\
1709 . \\
941 . \\
1228 .\end{array}$ \\
\hline 3 & $\begin{array}{l}219 . \\
220 . \\
221 . \\
222 . \\
223 . \\
224 . \\
225 . \\
226 .\end{array}$ & $\begin{array}{l}2.371 \\
2.743 \\
2.467 \\
3.207 \\
2.560 \\
1.248 \\
2.570 \\
1.589\end{array}$ & $\begin{array}{r}14.410 \\
11.290 \\
10.270 \\
11.770 \\
10.300 \\
4.109 \\
11.520 \\
6.146\end{array}$ & $\begin{array}{l}1332 . \\
1435 . \\
1551 . \\
1438 . \\
1246 . \\
1041 . \\
1202 . \\
1703 .\end{array}$ & $\begin{array}{l}.600 \\
.600 \\
.571 \\
.600 \\
.571 \\
.571 \\
.571 \\
.571\end{array}$ & $\begin{array}{r}2355 . \\
2356 . \\
650 . \\
2359 . \\
2134 . \\
1710^{\circ} . \\
939 . \\
1228 .\end{array}$ \\
\hline
\end{tabular}


Table 3. Summary of 1984 data: Raft station wind speed--(1) dally average wind speed at 1,2 and 3 meters above lake surface, (2) datly maximum and minimum wind speed at the level and the time they occurred. (continued)

\begin{tabular}{|c|c|c|c|c|c|c|}
\hline $\begin{array}{l}\text { HEIGHT OF } \\
\text { MEASUREMENT } \\
\text { AHOUE LAKE } \\
\text { SURFACE } \\
\text { (m) }\end{array}$ & $\begin{array}{l}\text { JUL IAN } \\
\text { IIAY }\end{array}$ & $\begin{array}{l}\text { IIAILY } \\
\text { AUERAGE } \\
\text { WINI } \\
\text { SFEEII } \\
(m I / h)\end{array}$ & $\begin{array}{l}\text { IIAILY } \\
\text { MAXIMUM } \\
\text { WINII } \\
\text { SFEEII } \\
(m i / h)\end{array}$ & $\begin{array}{l}\text { TIME OF } \\
\text { MAXIMUM } \\
\text { WINII } \\
\text { SFEEII } \\
\text { (h) }\end{array}$ & $\begin{array}{l}\text { IIAILY } \\
\text { MINIMUM } \\
\text { WINII } \\
\text { SFEEII } \\
(m i / h) \\
-----\end{array}$ & $\begin{array}{l}\text { TIME OF } \\
\text { MINIMUM } \\
\text { WINII } \\
\text { SFEEI } \\
\text { (h) }\end{array}$ \\
\hline 1 & $\begin{array}{l}227 . \\
228 . \\
229 . \\
230 . \\
231 . \\
232 . \\
233 . \\
234 .\end{array}$ & $\begin{array}{l}1.943 \\
2.946 \\
2.595 \\
6.115 \\
2.925 \\
1.779 \\
5.360 \\
3.063\end{array}$ & $\begin{array}{r}9.510 \\
17.490 \\
20.120 \\
26.860 \\
12.540 \\
24.990 \\
16.100\end{array}$ & $\begin{array}{r}1104 . \\
1009 . \\
2358 \\
840 \\
43 . \\
1253 . \\
941 .\end{array}$ & $\begin{array}{r}.571 \\
.571 \\
.571 \\
.600 \\
.571 \\
.571 \\
.571\end{array}$ & $\begin{array}{r}757 . \\
18377^{\circ} \\
20390^{\circ} \\
21390^{\circ} \\
854 . \\
734 . \\
32 .\end{array}$ \\
\hline 2 & $\begin{array}{l}227 . \\
228 . \\
229 . \\
230 . \\
231 . \\
232 . \\
233 . \\
234 .\end{array}$ & $\begin{array}{l}2.527 \\
3.350 \\
3.086 \\
6.504 \\
3.245 \\
2.235 \\
5.797 \\
3.455\end{array}$ & $\begin{array}{r}9.590 \\
18.400 \\
19.100 \\
25.530 \\
12.110 \\
25.670 \\
17.660\end{array}$ & $\begin{array}{r}1013 . \\
1015 . \\
2248 . \\
840 \\
43 . \\
1253 . \\
941 .\end{array}$ & $\begin{array}{l}.600 \\
.571 \\
.571 \\
.600 \\
.571 \\
.571 \\
.600\end{array}$ & $\begin{array}{r}23544^{\circ} \\
1837^{\circ} \\
2039^{\circ} \\
2117{ }^{\circ} \\
854{ }^{\circ} \\
732 . \\
2349 .\end{array}$ \\
\hline 3 & $\begin{array}{l}227 . \\
228 . \\
229 . \\
230 . \\
231 . \\
232 . \\
233 . \\
234 .\end{array}$ & $\begin{array}{l}2.498 \\
3.369 \\
3.060 \\
6.708 \\
3.308 \\
2.251 \\
5.956 \\
3.521\end{array}$ & $\begin{array}{l}11.090 \\
19.950 \\
18.710 \\
25.330 \\
11.150 \\
28.730 \\
18.650\end{array}$ & $\begin{array}{r}1230 . \\
1015 . \\
2358 . \\
840 . \\
1454 . \\
1403 . \\
941 .\end{array}$ & $\begin{array}{l}.600 \\
.571 \\
.571 \\
.600 \\
.571 \\
.571 \\
.600\end{array}$ & $\begin{array}{r}2350 . \\
805 . \\
1636 . \\
2117 . \\
854 . \\
732 . \\
2349 .\end{array}$ \\
\hline
\end{tabular}


Table 3. Summary of 1984 data: Raft station wind speed--(1) dally average wind speed at 1, 2 and 3 meters above lake surface, (2) daily maximum and minimum wind speed at the level and the time they occurred. (continued)

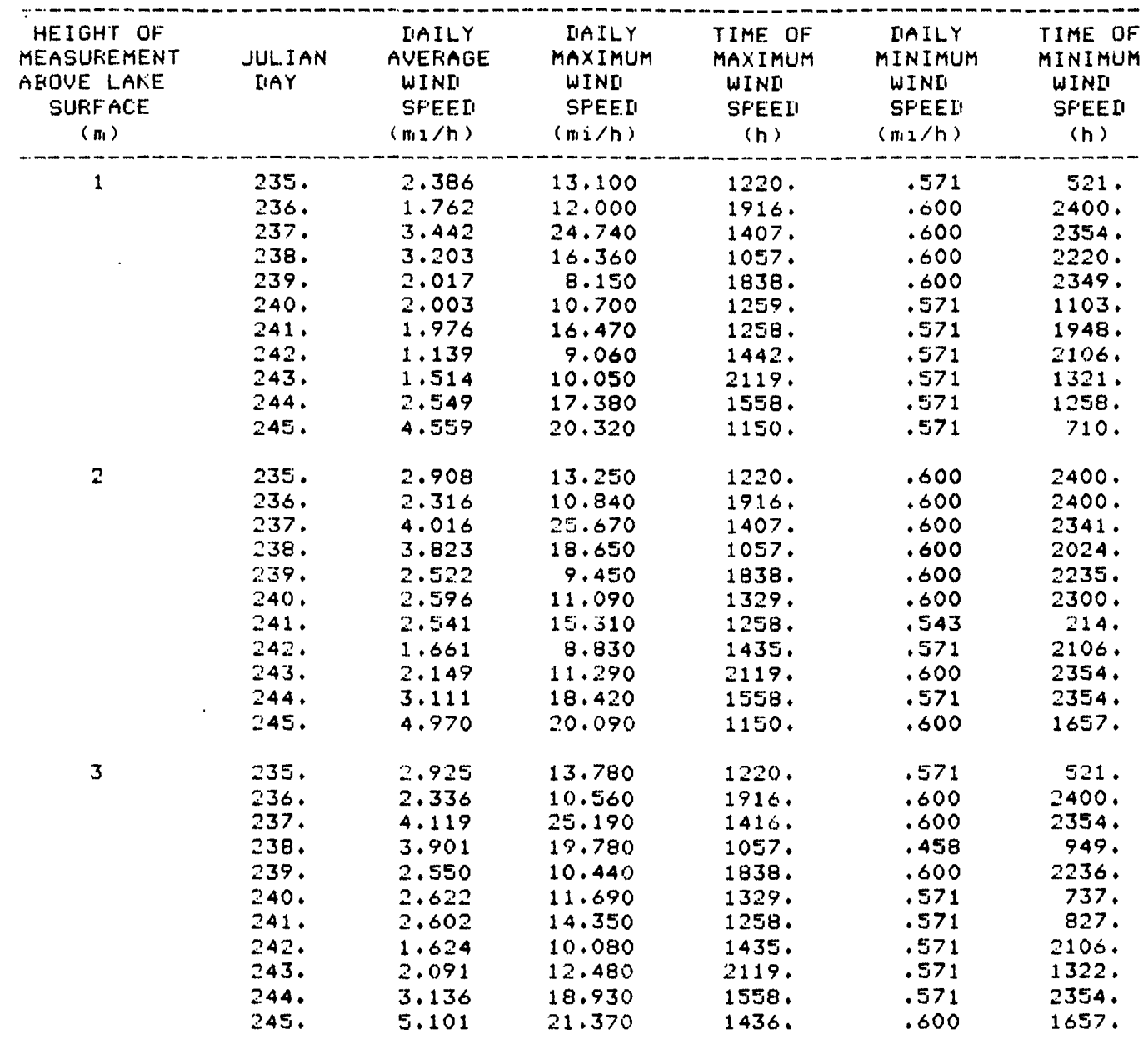


Table 3. Summary of 1984 data: Raft station wind speed--(1) dally average wind speed at 1,2 and 3 meters above lake surface, (2) daily maximum and minimum wind speed at the level and the time they occurred. (continued)

\begin{tabular}{|c|c|c|c|c|c|c|}
\hline $\begin{array}{l}\text { HEIGHT OF } \\
\text { MEASUKEMENT } \\
\text { AEOUE LAKE } \\
\text { SURFACE } \\
(m)\end{array}$ & $\begin{array}{l}\text { JULI IAN } \\
\text { IIAY }\end{array}$ & $\begin{array}{l}\text { IIAILY } \\
\text { AUERAGE } \\
\text { WINII } \\
\text { SFEEII } \\
\left(m_{2} / h\right)\end{array}$ & $\begin{array}{l}\text { IIAILY } \\
\text { MAXIMUM } \\
\text { WINII } \\
\text { SFEEII } \\
(m i / h)\end{array}$ & $\begin{array}{l}\text { TIME OF } \\
\text { MAXIMUM } \\
\text { WINII } \\
\text { SFEEII } \\
\text { (h) }\end{array}$ & $\begin{array}{l}\text { IIAILY } \\
\text { MINIMUM } \\
\text { WINII } \\
\text { SFEED } \\
(m i / h)\end{array}$ & $\begin{array}{l}\text { TIME OF } \\
\text { MINIMUM } \\
\text { WINII } \\
\text { SFEEII } \\
\text { (h) }\end{array}$ \\
\hline 1 & $\begin{array}{l}246 . \\
247 . \\
248 . \\
249 . \\
250 . \\
251 . \\
252 . \\
253 . \\
254 .\end{array}$ & $\begin{array}{r}4.976 \\
1.601 \\
.949 \\
3.551 \\
4.464 \\
3.199 \\
2.555 \\
2.155 \\
2.114\end{array}$ & $\begin{array}{r}21.200 \\
5.977 \\
7.307 \\
23.260 \\
22.870 \\
22.330 \\
13.420 \\
11.720 \\
13.900\end{array}$ & $\begin{array}{r}1107 . \\
618 . \\
1545 . \\
1803 . \\
927 . \\
1039 . \\
1110 . \\
1501 . \\
1404 .\end{array}$ & $\begin{array}{l}.050 \\
.571 \\
.543 \\
.571 \\
.600 \\
.600 \\
.571 \\
.571 \\
.571\end{array}$ & $\begin{array}{r}1437 . \\
17399^{\circ} \\
905 . \\
636 . \\
2357^{\circ} \\
2341 . \\
637 . \\
1239 . \\
51 .\end{array}$ \\
\hline 2 & $\begin{array}{l}246 . \\
247 . \\
248 . \\
249 . \\
250 . \\
251 . \\
252 . \\
253 . \\
254 .\end{array}$ & $\begin{array}{l}5.540 \\
2.010 \\
1.867 \\
4.120 \\
4.914 \\
3.647 \\
3.062 \\
2.746 \\
2.840\end{array}$ & $\begin{array}{r}20.830 \\
7.024 \\
7.505 \\
25.950 \\
25.640 \\
22.240 \\
13.640 \\
11.550 \\
14.120\end{array}$ & $\begin{array}{r}1440 . \\
36 . \\
1545 . \\
1803 . \\
902 . \\
1039 . \\
1110 . \\
1254 . \\
1404 .\end{array}$ & $\begin{array}{l}.600 \\
.571 \\
.305 \\
.571 \\
.600 \\
.600 \\
.571 \\
.571 \\
.571\end{array}$ & $\begin{array}{r}2359 . \\
1625 . \\
1029 . \\
636 . \\
2357 . \\
2340 . \\
835 . \\
405 . \\
51 .\end{array}$ \\
\hline 3 & $\begin{array}{l}246 . \\
247 . \\
248 . \\
249 . \\
250 . \\
251 . \\
252 . \\
253 . \\
254 .\end{array}$ & $\begin{array}{l}5.665 \\
2.032 \\
1.713 \\
4.124 \\
5.033 \\
3.749 \\
3.158 \\
2.776 \\
2.907\end{array}$ & $\begin{array}{r}21.760 \\
6.939 \\
7.703 \\
26.570 \\
26.600 \\
21.620 \\
13.440 \\
11.890 \\
14.430\end{array}$ & $\begin{array}{r}1106 . \\
36 . \\
1545^{\circ} \\
1803 . \\
902 . \\
1137^{\circ} \\
1110^{\circ} \\
1157 . \\
1404 .\end{array}$ & $\begin{array}{l}.600 \\
.175 \\
.571 \\
.571 \\
.600 \\
.571 \\
.571 \\
.571 \\
.571\end{array}$ & $\begin{array}{r}2350 . \\
1625 . \\
1246 . \\
514 . \\
2357 . \\
2100 . \\
840 . \\
405 . \\
51 .\end{array}$ \\
\hline
\end{tabular}


Table 3. Summary of 1984 data: Raft station wind speed--(1) daily average wind speed at 1,2 and 3 meters above lake surface, (2) daily maximum and minimum wind speed at the level and the time they occurred. (continued)

\begin{tabular}{|c|c|c|c|c|c|c|}
\hline $\begin{array}{c}\text { HEIGHT OF } \\
\text { MEASUREMENT } \\
\text { ABOUE LAKE } \\
\text { SUFFACE } \\
\left(n_{1}\right)\end{array}$ & $\begin{array}{c}\text { JUL I AN } \\
\text { IIAY }\end{array}$ & $\begin{array}{l}\text { IIAILY } \\
\text { AUERAGE } \\
\text { WINI! } \\
\text { SFEEI } \\
(m i / h)\end{array}$ & $\begin{array}{l}\text { IIAILY } \\
\text { MAXIMUM } \\
\text { WIND } \\
\text { SFEEI } \\
(m 1 / h)\end{array}$ & $\begin{array}{l}\text { TIME OF } \\
\text { MAXIMUM } \\
\text { WINI } \\
\text { SFEEI } \\
\text { (n) }\end{array}$ & $\begin{array}{l}\text { IIAILY } \\
\text { MINIMUM } \\
\text { WINI } \\
\text { SFEEI } \\
(m I / h)\end{array}$ & $\begin{array}{l}\text { TIME OF } \\
\text { MINIMUM } \\
\text { WINII } \\
\text { SFEEI } \\
\text { (h) }\end{array}$ \\
\hline 1 & $\begin{array}{l}255 . \\
256 . \\
257 . \\
258 . \\
259 . \\
260 . \\
261 . \\
262 .\end{array}$ & $\begin{array}{l}2.509 \\
5.118 \\
1.537 \\
3.446 \\
2.948 \\
4.093 \\
3.889 \\
2.175\end{array}$ & $\begin{array}{r}21.030 \\
30.710 \\
9.420 \\
18.820 \\
21.910 \\
19.020 \\
17.860 \\
15.080\end{array}$ & $\begin{array}{r}1628 . \\
1132 . \\
1056 . \\
1126 . \\
2334 . \\
316 . \\
955 . \\
1258 .\end{array}$ & $\begin{array}{l}.600 \\
.600 \\
.571 \\
.571 \\
.571 \\
.600 \\
.571 \\
.600\end{array}$ & $\begin{array}{r}2357 . \\
2400 . \\
1840 . \\
941 . \\
431 . \\
2356 . \\
1946 . \\
2359 .\end{array}$ \\
\hline 2 & $\begin{array}{l}255 . \\
256 . \\
257 . \\
258 . \\
259 . \\
260 . \\
261 . \\
262 .\end{array}$ & $\begin{array}{l}3.119 \\
5.724 \\
2.135 \\
4.034 \\
3.336 \\
4.473 \\
4.356 \\
2.752\end{array}$ & $\begin{array}{l}25.610 \\
32.550 \\
10.190 \\
16.610 \\
21.400 \\
19.670 \\
18.710 \\
15.230\end{array}$ & $\begin{array}{r}1628 . \\
1132 . \\
1104 . \\
1126 . \\
2334 . \\
316 . \\
955 . \\
1258 .\end{array}$ & $\begin{array}{l}.600 \\
.600 \\
.571 \\
.571 \\
.600 \\
.543 \\
.571 \\
.600\end{array}$ & $\begin{array}{r}2035 . \\
2331 . \\
1840 . \\
941 . \\
2044 . \\
803 . \\
354 . \\
2359 .\end{array}$ \\
\hline 3 & $\begin{array}{l}255 . \\
256 . \\
257 . \\
258 . \\
259 . \\
260 . \\
261 . \\
262 .\end{array}$ & $\begin{array}{l}3.196 \\
5.879 \\
2.067 \\
4.074 \\
3.391 \\
4.570 \\
4.440 \\
2.819\end{array}$ & $\begin{array}{l}26.690 \\
33.710 \\
10.980 \\
17.040 \\
19.700 \\
17.150 \\
18.990 \\
15.250\end{array}$ & $\begin{array}{r}1628 . \\
1132 . \\
1056 . \\
1308 . \\
2334 . \\
1258 . \\
955 . \\
1258 .\end{array}$ & $\begin{array}{l}.571 \\
.571 \\
.571 \\
.571 \\
.571 \\
.571 \\
.543 \\
.571\end{array}$ & $\begin{array}{r}945 . \\
1102 . \\
1840^{\circ} \\
941 . \\
2012^{\circ} \\
2125 . \\
757 . \\
740 .\end{array}$ \\
\hline
\end{tabular}

\begin{tabular}{|c|c|c|c|c|c|c|}
\hline $\begin{array}{c}\text { HEIGHT OF } \\
\text { MEASUFEMENT } \\
\text { ABOUE LAKE } \\
\text { SUFFACE } \\
(m)\end{array}$ & $\begin{array}{l}\text { JUL I AN } \\
\text { IIAY }\end{array}$ & $\begin{array}{l}\text { IUAILY } \\
\text { AUERAGE } \\
\text { WINII } \\
\text { SFEEI } \\
(\Pi i / h)\end{array}$ & $\begin{array}{l}\text { DAILY } \\
\text { MAXIMUM } \\
\text { WINII } \\
\text { SFEEII } \\
\text { (MiIh) }\end{array}$ & $\begin{array}{l}\text { TIME OF } \\
\text { MAXIMUM } \\
\text { WINII } \\
\text { SFEEII } \\
(h)\end{array}$ & $\begin{array}{l}\text { IIAILY } \\
\text { MINIMUM } \\
\text { WINII } \\
\text { SFEEII } \\
\text { (mi/h) }\end{array}$ & $\begin{array}{l}\text { TIME OF } \\
\text { MINIMUM } \\
\text { WINI } \\
\text { SFEEI } \\
\text { (h) }\end{array}$ \\
\hline 1 & $\begin{array}{l}263 . \\
264 . \\
265 . \\
266 . \\
267 .\end{array}$ & $\begin{array}{l}2.208 \\
3.683 \\
5.600 \\
1.822 \\
1.639\end{array}$ & $\begin{array}{l}17.350 \\
29.570 \\
25.870 \\
12.820 \\
11.350\end{array}$ & $\begin{array}{l}1357 \\
1755 \\
1104 \\
1219 \\
2323 .\end{array}$ & $\begin{array}{r}.571 \\
.571 \\
.571 \\
.571 \\
.571\end{array}$ & $\begin{array}{r}2234 . \\
800 . \\
321 . \\
1725 . \\
2135 .\end{array}$ \\
\hline 2 & $\begin{array}{l}263 . \\
264 . \\
265 . \\
266 . \\
267 .\end{array}$ & $\begin{array}{l}2.819 \\
4.340 \\
6.160 \\
2.715 \\
2.248\end{array}$ & $\begin{array}{l}17.240 \\
30.450 \\
24.540 \\
12.910 \\
12.710\end{array}$ & $\begin{array}{l}1357 \\
1753 \\
1104 . \\
1219 . \\
1445 .\end{array}$ & $\begin{array}{l}.571 \\
.571 \\
.600 \\
.571 \\
.571\end{array}$ & $\begin{array}{r}2234 \\
653 \\
2400 \\
827 \\
2135\end{array}$ \\
\hline 3 & $\begin{array}{l}263 . \\
264 . \\
265 . \\
260 . \\
267 .\end{array}$ & $\begin{array}{l}2.857 \\
4.460 \\
0.372 \\
2.741 \\
2.212\end{array}$ & $\begin{array}{l}20.210 \\
29.520 \\
23.210 \\
12.960 \\
12.540\end{array}$ & $\begin{array}{l}135 \% \\
1755 . \\
1104 . \\
121 \% \\
1445 .\end{array}$ & $\begin{array}{l}.600 \\
.571 \\
.600 \\
.600 \\
.571\end{array}$ & $\begin{array}{r}2358 \\
653 . \\
2400 . \\
2154 . \\
2135 .\end{array}$ \\
\hline
\end{tabular}


Table 3. Summary of 1984 data: Raft station wind speed--(1) dally average wind speed at 1,2 and 3 meters above lake surface, (2) daily maximum and mintmum wind speed at the level and the time they occurred. (continued)

\begin{tabular}{|c|c|c|c|c|c|c|}
\hline $\begin{array}{l}\text { HEIGHT OF } \\
\text { MEASUFEMENT } \\
\text { AEOUE LAKE } \\
\text { SUFFACE } \\
\left(m_{1}\right)\end{array}$ & $\begin{array}{l}\text { JUL I IAN } \\
\text { IIAY }\end{array}$ & $\begin{array}{l}\text { IIAILY } \\
\text { AUERAGE } \\
\text { WINII } \\
\text { SFEEII } \\
(m 11 / n)\end{array}$ & $\begin{array}{l}\text { IIAILY } \\
\text { MAXIMUM } \\
\text { WINII } \\
\text { SFEEI } \\
\left(m_{1} / h\right)\end{array}$ & $\begin{array}{l}\text { TIME OF } \\
\text { MAXIMUM } \\
\text { WINI } \\
\text { SFEEI } \\
\text { (h) }\end{array}$ & $\begin{array}{l}\text { IIAILY } \\
\text { MINIMUM } \\
\text { WINII } \\
\text { SFEEI } \\
(m i / h)\end{array}$ & $\begin{array}{l}\text { TIME OF } \\
\text { MINIMUM } \\
\text { WINII } \\
\text { SFEEII } \\
\text { (h) }\end{array}$ \\
\hline 1 & $\begin{array}{l}268 . \\
269 . \\
270 . \\
271 . \\
272 . \\
273 . \\
274 . \\
275 . \\
276 . \\
277 . \\
278 . \\
279 . \\
280 . \\
281 .\end{array}$ & $\begin{array}{l}2.994 \\
1.298 \\
5.304 \\
4.046 \\
2.212 \\
1.503 \\
1.622 \\
2.122 \\
4.727 \\
2.845 \\
6.025 \\
7.505 \\
6.881 \\
1.427\end{array}$ & $\begin{array}{r}17.740 \\
10.440 \\
28.390 \\
23.240 \\
14.010 \\
6.401 \\
12.200 \\
9.370 \\
18.310 \\
28.870 \\
24.710 \\
33.930 \\
25.190 \\
8.260\end{array}$ & $\begin{array}{r}1147 . \\
1256 . \\
1348 . \\
130 . \\
1334 . \\
1158 . \\
1044 . \\
1234 . \\
805 . \\
125 . \\
923 . \\
540 . \\
448 . \\
1335 .\end{array}$ & $\begin{array}{l}.600 \\
.571 \\
.571 \\
.571 \\
.571 \\
.571 \\
.571 \\
.571 \\
.571 \\
.571 \\
.600 \\
.600 \\
.600 \\
.571\end{array}$ & $\begin{array}{r}2353 . \\
2201 . \\
1647 . \\
1659 . \\
2159 . \\
752 . \\
1012 . \\
345 . \\
2032 . \\
1407 . \\
2400 . \\
2245 . \\
2310 . \\
1657 .\end{array}$ \\
\hline 2 & $\begin{array}{l}268 . \\
269 . \\
270 . \\
271 . \\
272 . \\
273 . \\
274 . \\
275 . \\
276 . \\
277 . \\
278 . \\
279 . \\
280 . \\
281 .\end{array}$ & $\begin{array}{l}3.957 \\
1.927 \\
5.947 \\
4.660 \\
3.067 \\
2.172 \\
2.331 \\
2.715 \\
5.136 \\
3.655 \\
6.725 \\
8.060 \\
7.307 \\
2.018\end{array}$ & $\begin{array}{r}20.090 \\
11.040 \\
28.900 \\
22.810 \\
15.680 \\
6.627 \\
12.230 \\
10.640 \\
18.230 \\
30.480 \\
29.910 \\
31.020 \\
25.730 \\
8.490\end{array}$ & $\begin{array}{r}1140 . \\
1256 . \\
1348 . \\
130 . \\
1334 . \\
1158 . \\
1044 . \\
1522 . \\
2108 . \\
40 . \\
923 . \\
540 . \\
346 . \\
1335 .\end{array}$ & $\begin{array}{l}.600 \\
.571 \\
.600 \\
.600 \\
.571 \\
.571 \\
.571 \\
.600 \\
.571 \\
.600 \\
.600 \\
.600 \\
.600 \\
.571\end{array}$ & $\begin{array}{r}2341 . \\
2203 . \\
2319 . \\
2345 . \\
1825 . \\
753 . \\
901 . \\
2331 . \\
2022 . \\
2315 . \\
2400 . \\
2241 . \\
2306 . \\
347 .\end{array}$ \\
\hline 3 & $\begin{array}{l}268 . \\
269 . \\
270 . \\
271 . \\
272 . \\
273 . \\
274 . \\
275 . \\
276 . \\
277 . \\
278 . \\
279 . \\
280 . \\
281 .\end{array}$ & $\begin{array}{l}3.966 \\
1.873 \\
6.122 \\
4.711 \\
3.030 \\
2.042 \\
2.177 \\
2.634 \\
5.271 \\
3.681 \\
6.969 \\
8.310 \\
7.559 \\
2.050\end{array}$ & $\begin{array}{r}21.510 \\
10.750 \\
26.660 \\
24.230 \\
15.910 \\
6.769 \\
12.200 \\
10.870 \\
19.390 \\
30.710 \\
28.440 \\
31.100 \\
26.180 \\
8.320\end{array}$ & $\begin{array}{r}1140 . \\
1437 . \\
1348 . \\
120 . \\
1334 . \\
1158 . \\
1154 . \\
1234 . \\
2249 . \\
40 . \\
923 . \\
540^{\circ} . \\
936 . \\
1335 .\end{array}$ & $\begin{array}{l}.600 \\
.571 \\
.600 \\
.571 \\
.571 \\
.571 \\
.571 \\
.571 \\
.571 \\
.571 \\
.600 \\
.600 \\
.600 \\
.571\end{array}$ & $\begin{array}{r}2347 . \\
2203 . \\
2327 . \\
244 . \\
2158 . \\
753 . \\
2120 . \\
2239 . \\
2040 . \\
1410 . \\
2400 . \\
2241 . \\
2306 . \\
921 .\end{array}$ \\
\hline
\end{tabular}


Table 3. Summary of 1984 data: Raft station wind speed--(1) daily average wind speed at 1,2 and 3 meters above lake surface, (2) daily maximum and minimum wind speed at the level and the time they occurred. (continued)

\begin{tabular}{|c|c|c|c|c|c|c|}
\hline $\begin{array}{l}\text { HEIGHT OF } \\
\text { MEASUFEMENT } \\
\text { AEOUE LAKE } \\
\text { SUFFACE } \\
(m)\end{array}$ & $\begin{array}{l}\text { IUL. IAN } \\
\text { IIAY }\end{array}$ & $\begin{array}{l}\text { IIAILY } \\
\text { AUERAGE } \\
\text { WINII } \\
\text { SFEEII } \\
(m I / h)\end{array}$ & $\begin{array}{l}\text { IIAILY } \\
\text { MAXIMUM } \\
\text { WINII } \\
\text { SFEEI } \\
\text { (mi/h) }\end{array}$ & $\begin{array}{l}\text { TIME OF } \\
\text { MAXIMUM } \\
\text { WINII } \\
\text { SFEEI } \\
\text { (h) }\end{array}$ & $\begin{array}{l}\text { IIAILY } \\
\text { MINIMUM } \\
\text { WINI } \\
\text { SFEEII } \\
(\mathrm{mi} / \mathrm{h})\end{array}$ & $\begin{array}{l}\text { TIME OF } \\
\text { MINIMUM } \\
\text { WINI } \\
\text { SFEEII } \\
\text { (h) }\end{array}$ \\
\hline 1 & $\begin{array}{l}282 . \\
283 . \\
284 . \\
285 . \\
286 . \\
287 . \\
288 .\end{array}$ & $\begin{array}{l}1.187 \\
2.077 \\
1.959 \\
3.362 \\
2.510 \\
1.777 \\
1.908\end{array}$ & $\begin{array}{r}8.830 \\
10.980 \\
14.180 \\
20.550 \\
18.570 \\
16.100 \\
9.370\end{array}$ & $\begin{array}{r}1345 . \\
1924 . \\
1908 . \\
1539 . \\
105 . \\
1451 . \\
1205 .\end{array}$ & $\begin{array}{r}.571 \\
.571 \\
.571 \\
.571 \\
.571 \\
.571 \\
.571\end{array}$ & $\begin{array}{l}2210 . \\
1813 . \\
1258 . \\
2235 . \\
2250 . \\
2308 . \\
2057 .\end{array}$ \\
\hline 2 & $\begin{array}{l}282 . \\
283 . \\
284 . \\
285^{\circ} \\
286 . \\
287 . \\
288 .\end{array}$ & $\begin{array}{l}1.740 \\
2.606 \\
2.506 \\
3.968 \\
3.006 \\
2.171 \\
2.457\end{array}$ & $\begin{array}{r}9.030 \\
11.490 \\
13.810 \\
20.580 \\
19.610 \\
15.200 \\
9.200\end{array}$ & $\begin{array}{l}1423 . \\
1924 . \\
1930 . \\
1426 . \\
105 . \\
1451 . \\
1205 .\end{array}$ & $\begin{array}{l}.571 \\
.571 \\
.571 \\
.571 \\
.571 \\
.571 \\
.571\end{array}$ & $\begin{array}{r}2210 . \\
917 . \\
1245 . \\
2235 . \\
2028 . \\
1902 . \\
2334 .\end{array}$ \\
\hline 3 & $\begin{array}{l}282^{\circ} \\
283 . \\
284^{\circ} \\
285^{\circ} \\
286^{\circ} \\
287^{\circ} \\
280^{\circ}\end{array}$ & $\begin{array}{l}1.751 \\
2.575 \\
2.480 \\
4.008 \\
3.001 \\
2.025 \\
2.350\end{array}$ & $\begin{array}{r}9.450 \\
12.200 \\
14.720 \\
21.450 \\
19.360 \\
14.180 \\
11.040\end{array}$ & $\begin{array}{r}1421 . \\
1855 . \\
1930 . \\
1426 . \\
105 . \\
1451 . \\
1216 .\end{array}$ & $\begin{array}{l}.571 \\
.571 \\
.571 \\
.571 \\
.571 \\
.571 \\
.571\end{array}$ & $\begin{array}{l}2210^{\circ} \\
2109 . \\
1257 . \\
2235 . \\
2250 . \\
2308 . \\
2334 .\end{array}$ \\
\hline
\end{tabular}

\begin{tabular}{|c|c|c|c|c|c|c|}
\hline $\begin{array}{l}\text { HEIGHT OF } \\
\text { MEASUFEMENT } \\
\text { AEOUE LALE } \\
\text { SUFFACE. } \\
\text { (mi) }\end{array}$ & $\begin{array}{l}\text { JULI AN } \\
\text { IIAY }\end{array}$ & $\begin{array}{l}\text { IIAILY } \\
\text { AUERIAGE } \\
\text { WINII } \\
\text { SFEEII } \\
(m i / n)\end{array}$ & $\begin{array}{l}\text { IIAILY } \\
\text { MAXIMUM } \\
\text { WINI } \\
\text { SFEEI } \\
\left(n_{1} 1 / h\right)\end{array}$ & $\begin{array}{l}\text { TIME OF } \\
\text { MAXIMUM } \\
\text { WINII } \\
\text { SFEEII } \\
\text { (h) }\end{array}$ & $\begin{array}{l}\text { IIAILY } \\
\text { MINIMUM } \\
\text { WINII } \\
\text { SFEEI } \\
(m i / h)\end{array}$ & $\begin{array}{l}\text { TIME OF } \\
\text { MINIMUM } \\
\text { WINII } \\
\text { SFEEI } \\
\text { (h) }\end{array}$ \\
\hline 1 & $\begin{array}{l}289 . \\
290 . \\
291 . \\
292 . \\
293 . \\
294 . \\
295 . \\
296 . \\
297 . \\
298 .\end{array}$ & $\begin{array}{l}1.067 \\
1.846 \\
1.732 \\
1.818 \\
1.132 \\
2.737 \\
1.018 \\
2.687 \\
2.027 \\
1.509\end{array}$ & $\begin{array}{r}6.910 \\
10.100 \\
10.250 \\
8.600 \\
11.720 \\
13.670 \\
6.344 \\
16.750 \\
15.370 \\
9.280\end{array}$ & $\begin{array}{l}1345 . \\
1603 . \\
1033 . \\
1323 . \\
1358 . \\
1405 . \\
1507 . \\
1515 . \\
1412 . \\
1425 .\end{array}$ & $\begin{array}{l}.571 \\
.571 \\
.571 \\
.500 \\
.571 \\
.571 \\
.571 \\
.571 \\
.571 \\
.571\end{array}$ & $\begin{array}{l}2200^{\circ} \\
2047 . \\
2056 . \\
2400 . \\
1939 . \\
608 . \\
2158 . \\
2113 . \\
2039 . \\
1548 .\end{array}$ \\
\hline 2 & $\begin{array}{l}289 . \\
290 . \\
291 . \\
292 . \\
293 . \\
294 . \\
295 . \\
296 . \\
297 . \\
298 .\end{array}$ & $\begin{array}{l}1.738 \\
2.247 \\
2.113 \\
2.312 \\
1.597 \\
3.227 \\
1.619 \\
3.133 \\
2.526 \\
2.097\end{array}$ & $\begin{array}{r}7.618 \\
10.700 \\
9.960 \\
9.280 \\
11.410 \\
13.950 \\
6.033 \\
17.150 \\
15.340 \\
9.570\end{array}$ & $\begin{array}{l}1114 . \\
1603 . \\
1033 . \\
1355 . \\
1358 . \\
1413 . \\
1506 . \\
1515 . \\
1412 . \\
1425 .\end{array}$ & $\begin{array}{l}.571 \\
.571 \\
.571 \\
.571 \\
.571 \\
.571 \\
.571 \\
.571 \\
.571 \\
.571\end{array}$ & $\begin{array}{r}1945 . \\
2320 . \\
2056 . \\
2313 . \\
1455 . \\
712 . \\
2201 . \\
2113 . \\
2036 . \\
1850 .\end{array}$ \\
\hline 3 & $\begin{array}{l}289 . \\
290 . \\
291 . \\
292 . \\
293 . \\
294 . \\
295 . \\
296 . \\
297 . \\
298 .\end{array}$ & $\begin{array}{l}1.663 \\
2.189 \\
2.068 \\
2.284 \\
1.575 \\
3.259 \\
1.501 \\
3.053 \\
2.300 \\
1.738\end{array}$ & $\begin{array}{r}7.420 \\
11.600 \\
10.470 \\
9.880 \\
11.150 \\
15.990 \\
5.807 \\
17.550 \\
14.460 \\
9.510\end{array}$ & $\begin{array}{l}1345 . \\
1233 . \\
1033 . \\
1544 . \\
1358 . \\
1413 . \\
1506 . \\
1515 . \\
1412 . \\
1425 .\end{array}$ & $\begin{array}{l}.571 \\
.571 \\
.571 \\
.571 \\
.571 \\
.571 \\
.571 \\
.571 \\
.571 \\
.571\end{array}$ & $\begin{array}{r}1945 . \\
2320 . \\
2056 . \\
2332 . \\
1939 . \\
712 . \\
2218 . \\
2113 . \\
2041 . \\
1850 .\end{array}$ \\
\hline
\end{tabular}


Table 3. Summary of 1984 data: Raft station wind speed--(1) dafly average wind speed at 1,2 and 3 meters above lake surface, (2) dally maximum and minimum wind speed at the level and the time they occurred. (continued)

\begin{tabular}{|c|c|c|c|c|c|c|}
\hline $\begin{array}{c}\text { HEIGHT OF } \\
\text { MEASUREMENT } \\
\text { ABDUE LAKE } \\
\text { SUFFACE } \\
\left(m_{1}\right)\end{array}$ & $\begin{array}{l}\text { JUL. IAN } \\
\text { IIAY }\end{array}$ & $\begin{array}{l}\text { IIAILY } \\
\text { AUERAGE } \\
\text { WINI } \\
\text { SFEEII } \\
(n+i / h)\end{array}$ & $\begin{array}{l}\text { IIAILY } \\
\text { MAXIMUM } \\
\text { WINII } \\
\text { SFEEII } \\
(m 1 / h)\end{array}$ & $\begin{array}{l}\text { TIME OF } \\
\text { MAXIMUM } \\
\text { WINII } \\
\text { SFEEII } \\
(h)\end{array}$ & $\begin{array}{l}\text { IIAILY } \\
\text { MINIMUM } \\
\text { WINII } \\
\text { SPEEII } \\
(m I / h)\end{array}$ & $\begin{array}{l}\text { TIME OF } \\
\text { MINIMUM } \\
\text { WINII } \\
\text { SFE.EII } \\
\text { (h) }\end{array}$ \\
\hline 1 & $\begin{array}{l}299 . \\
300 . \\
301 . \\
302 . \\
303 . \\
304 .\end{array}$ & $\begin{array}{r}1.959 \\
1.402 \\
1.449 \\
.829 \\
4.037 \\
2.147\end{array}$ & $\begin{array}{r}12.760 \\
9.480 \\
6.627 \\
4.675 \\
20.740 \\
11.410\end{array}$ & $\begin{array}{r}1144 . \\
236 . \\
1427 . \\
208 . \\
538 . \\
1126 .\end{array}$ & $\begin{array}{r}.571 \\
.600 \\
.571 \\
.571 \\
.600 \\
.571\end{array}$ & $\begin{array}{r}1949 . \\
2400 . \\
1830 . \\
2044 . \\
2359 . \\
913 .\end{array}$ \\
\hline 2 & $\begin{array}{l}299 . \\
300 . \\
301 . \\
302 . \\
303 . \\
304 .\end{array}$ & $\begin{array}{l}2.448 \\
1.901 \\
2.064 \\
1.218 \\
4.490 \\
2.419\end{array}$ & $\begin{array}{r}11.940 \\
9.510 \\
7.307 \\
4.901 \\
23.070 \\
13.020\end{array}$ & $\begin{array}{r}1207 . \\
236 . \\
1609 . \\
1414 . \\
1058 . \\
1126 .\end{array}$ & $\begin{array}{l}.571 \\
.600 \\
.571 \\
.571 \\
.600 \\
.571\end{array}$ & $\begin{array}{l}1949 . \\
2352 . \\
1820^{\circ} \\
2044 . \\
2352 . \\
2346 .\end{array}$ \\
\hline 3 & $\begin{array}{l}299 . \\
300 . \\
301 . \\
302 . \\
303 . \\
304 .\end{array}$ & $\begin{array}{l}2.152 \\
1.621 \\
1.855 \\
1: 035 \\
4.477 \\
2.295\end{array}$ & $\begin{array}{r}12.990 \\
8.630 \\
6.967 \\
5.297 \\
24.030 \\
13.610\end{array}$ & $\begin{array}{r}1207 . \\
236 . \\
1609 . \\
1414 . \\
537 . \\
1126 .\end{array}$ & $\begin{array}{r}.571 \\
.600 \\
.571 \\
.571 \\
.486 \\
.571\end{array}$ & $\begin{array}{l}1949 . \\
2400 . \\
1818 . \\
20440^{\circ} \\
7260^{\circ} \\
2348 .\end{array}$ \\
\hline
\end{tabular}

\begin{tabular}{|c|c|c|c|c|c|c|}
\hline $\begin{array}{l}\text { HEIGHT OF } \\
\text { MEASUREMENT } \\
\text { ABOUE LAKE } \\
\text { SURFACE } \\
(m)\end{array}$ & $\begin{array}{l}\text { JUL I AN } \\
\text { IIAY }\end{array}$ & $\begin{array}{l}\text { IIAILY } \\
\text { AUEFIAGE } \\
\text { WINII } \\
\text { SPEEII } \\
(m 1 / n)\end{array}$ & $\begin{array}{l}\text { IIAILY } \\
\text { MAXIMUM } \\
\text { WINII } \\
\text { SFEEII } \\
(n 1 / h)\end{array}$ & $\begin{array}{l}\text { TIME OF } \\
\text { MAXIMUM } \\
\text { WINII } \\
\text { SFEEII } \\
\text { (h) }\end{array}$ & $\begin{array}{l}\text { IIAILY } \\
\text { MINIMUM } \\
\text { WINII } \\
\text { SFEEII } \\
(m i / h)\end{array}$ & $\begin{array}{l}\text { TIME OF } \\
\text { MINIMUM } \\
\text { WINI! } \\
\text { SFEEI } \\
\text { (h) }\end{array}$ \\
\hline 1 & $\begin{array}{l}305 . \\
306 . \\
307 . \\
308 . \\
309 .\end{array}$ & $\begin{array}{l}3.473 \\
2.671 \\
5.009 \\
3.367 \\
2.324\end{array}$ & $\begin{array}{l}22.390 \\
13.440 \\
27.540 \\
18.590 \\
12.930\end{array}$ & $\begin{array}{r}952 . \\
1219 . \\
1503 . \\
23 \% \\
1217\end{array}$ & $\begin{array}{l}.600 \\
.571 \\
.571 \\
.600 \\
.571\end{array}$ & $\begin{array}{r}2351 . \\
851 . \\
2104 . \\
2354 . \\
418 .\end{array}$ \\
\hline 2 & $\begin{array}{l}305 . \\
300 . \\
307 . \\
308 . \\
309 .\end{array}$ & $\begin{array}{l}3.966 \\
3.043 \\
5.399 \\
3.730 \\
2.649\end{array}$ & $\begin{array}{l}22.580 \\
13.560 \\
30.420 \\
17.860 \\
12.820\end{array}$ & $\begin{array}{r}952 . \\
1217 . \\
1503 . \\
232 . \\
1217 .\end{array}$ & $\begin{array}{l}.571 \\
.571 \\
.600 \\
.600 \\
.571\end{array}$ & $\begin{array}{r}819 . \\
852 . \\
2210 . \\
2354 . \\
346 .\end{array}$ \\
\hline 3 & $\begin{array}{l}305 . \\
306 . \\
307 . \\
308 . \\
309 .\end{array}$ & $\begin{array}{l}3.964 \\
3.073 \\
5.506 \\
3.747 \\
2.636\end{array}$ & $\begin{array}{l}22.920 \\
13.330 \\
30.760 \\
17.910 \\
13.300\end{array}$ & $\begin{array}{r}1545 . \\
1219 . \\
1503 . \\
232 . \\
1200 .\end{array}$ & $\begin{array}{l}.571 \\
.571 \\
.600 \\
.571 \\
.571\end{array}$ & $\begin{array}{r}819 . \\
901 . \\
2210 . \\
2227 . \\
418 .\end{array}$ \\
\hline
\end{tabular}


Table 3. Summary of 1984 data: Raft station wind speed--(1) daily average wind speed at 1,2 and 3 meters above lake surface, (2) daily maximum and minimum wind speed at the level and the time they occurred. (continued)

\begin{tabular}{|c|c|c|c|c|c|c|}
\hline $\begin{array}{c}\text { HEIGHT OF } \\
\text { MEASUKEMENT } \\
\text { AEOUE LAKE } \\
\text { SUFFACE. } \\
(m)\end{array}$ & $\underset{\text { IIAY I AN }}{J U L}$ & $\begin{array}{l}\text { IIAILY } \\
\text { AUEFAGE } \\
\text { WINLI } \\
\text { SFEEI } \\
(m 1 / h)\end{array}$ & $\begin{array}{l}\text { LIAILY } \\
\text { MAXIMUM } \\
\text { WINII } \\
\text { SFEEII } \\
(m i / h)\end{array}$ & $\begin{array}{l}\text { TIME QF } \\
\text { MAXIMUM } \\
\text { WINI } \\
\text { SFEEI } \\
\text { (h) }\end{array}$ & $\begin{array}{l}\text { IIAILY } \\
\text { MINIMUH } \\
\text { WINII } \\
\text { SFEEII } \\
\left(m_{2} / h\right)\end{array}$ & $\begin{array}{l}\text { TIME OF } \\
\text { MINIMUM } \\
\text { WINII } \\
\text { SFEEII } \\
\text { (h) }\end{array}$ \\
\hline$--m----m-m$ & $---m$ & $-x-m-m--$ & ------- & $--\infty-\infty$ & $---m-\infty$ & ------ \\
\hline 1 & $\begin{array}{l}310 . \\
311 . \\
312 . \\
313 . \\
314 . \\
315 . \\
316 . \\
317 . \\
318 .\end{array}$ & $\begin{array}{r}1.234 \\
5.345 \\
7.022 \\
2.185 \\
1.367 \\
.859 \\
.852 \\
2.192 \\
6.019\end{array}$ & $\begin{array}{r}10.900 \\
21.790 \\
23.720 \\
11.120 \\
5.354 \\
5.694 \\
4.477 \\
15.450 \\
25.920\end{array}$ & $\begin{array}{l}1751 . \\
1018 . \\
1141 . \\
1241 . \\
1206 . \\
1043 . \\
1639 . \\
2256 . \\
1459 .\end{array}$ & $\begin{array}{r}.571 \\
.571 \\
.600 \\
.600 \\
.571 \\
.571 \\
.571 \\
.600 \\
.571\end{array}$ & $\begin{array}{r}2215^{\circ} \\
2210^{\circ} \\
2340^{\circ} \\
2353 . \\
19011^{\circ} \\
742^{\circ} \\
1100^{\circ} \\
23590^{\circ} \\
2349 .\end{array}$ \\
\hline 2 & $\begin{array}{l}310 . \\
311 . \\
312 . \\
313 . \\
314 . \\
315 . \\
316 . \\
317 . \\
318 .\end{array}$ & $\begin{array}{r}1.465 \\
5.751 \\
7.355 \\
2.378 \\
1.387 \\
.878 \\
.843 \\
2.338 \\
6.462\end{array}$ & $\begin{array}{r}11.380 \\
22.610 \\
24.590 \\
11.660 \\
5.411 \\
6.090 \\
4.420 \\
17.090 \\
23.490\end{array}$ & $\begin{array}{l}1839 . \\
1018 . \\
1141 . \\
1222 . \\
1206 . \\
1043 . \\
1633 . \\
2256 . \\
1459 .\end{array}$ & $\begin{array}{l}.571 \\
.571 \\
.600 \\
.571 \\
.571 \\
.571 \\
.571 \\
.600 \\
.600\end{array}$ & $\begin{array}{r}2215 . \\
2210^{\circ} \\
2339 . \\
841 . \\
1858 . \\
811^{\circ} \\
1104 . \\
2323 . \\
1757 .\end{array}$ \\
\hline 3 & $\begin{array}{l}310 . \\
311 . \\
312 . \\
313 . \\
314 . \\
315 . \\
316 . \\
317 . \\
318 .\end{array}$ & $\begin{array}{r}1.433 \\
5.955 \\
7.539 \\
2.548 \\
1.474 \\
.925 \\
.865 \\
2.417 \\
6.736\end{array}$ & $\begin{array}{r}11.260 \\
22.730 \\
24.760 \\
11.920 \\
5.580 \\
6.033 \\
4.222 \\
17.070 \\
25.240\end{array}$ & $\begin{array}{r}1751 . \\
1018 . \\
542 . \\
1152 . \\
1048 . \\
1043 . \\
1639 . \\
2256 . \\
1459 .\end{array}$ & $\begin{array}{l}.571 \\
.571 \\
.600 \\
.571 \\
.571 \\
.571 \\
.571 \\
.600 \\
.571\end{array}$ & $\begin{array}{r}2215 . \\
2210^{\circ} \\
23390^{\circ} \\
841 . \\
19011^{\circ} \\
811^{\circ} \\
1219 . \\
2323 . \\
2345 .\end{array}$ \\
\hline
\end{tabular}

\begin{tabular}{|c|c|c|c|c|c|c|}
\hline $\begin{array}{l}\text { HEIGHT OF } \\
\text { MEASUREMENT } \\
\text { AEOUE LAKE } \\
\text { SURFACE } \\
\text { (m) }\end{array}$ & $\begin{array}{l}\text { JUL I AN } \\
\text { LIAY }\end{array}$ & $\begin{array}{l}\text { LIAILY } \\
\text { AUEFAGE. } \\
\text { WINI! } \\
\text { SFEEII } \\
(m i / h)\end{array}$ & $\begin{array}{l}\text { IIAILY } \\
\text { MAXIMUM } \\
\text { WINLI } \\
\text { SFEEII } \\
(m i / h)\end{array}$ & $\begin{array}{l}\text { TIME OF } \\
\text { MAXIMUM } \\
\text { WINII } \\
\text { SFEEII } \\
\text { (h) }\end{array}$ & $\begin{array}{l}\text { IIAILY } \\
\text { MINIMUM } \\
\text { WINII } \\
\text { SFEEII } \\
(\pi i / h)\end{array}$ & $\begin{array}{l}\text { TIME OF } \\
\text { MINIMUM } \\
\text { WINII } \\
\text { SFEEI } \\
\text { (h) }\end{array}$ \\
\hline 1 & $\begin{array}{l}319 . \\
320 . \\
321 . \\
322 . \\
323 .\end{array}$ & $\begin{array}{r}10.200 \\
2.306 \\
3.428 \\
7.520 \\
2.599\end{array}$ & $\begin{array}{l}39.560 \\
20.770 \\
19.410 \\
31.240 \\
14.600\end{array}$ & $\begin{array}{r}2058 . \\
43 . \\
1421 . \\
1646 . \\
304 .\end{array}$ & $\begin{array}{l}.571 \\
.571 \\
.571 \\
.600 \\
.600\end{array}$ & $\begin{array}{l}247 . \\
1901 . \\
1604 . \\
2348 . \\
2258 .\end{array}$ \\
\hline 2 & $\begin{array}{l}319 . \\
320 . \\
321 . \\
322 . \\
323 .\end{array}$ & $\begin{array}{r}10.850 \\
2.802 \\
3.734 \\
7.933 \\
2.982\end{array}$ & $\begin{array}{l}44.830 \\
25.610 \\
20.260 \\
31.730 \\
15.510\end{array}$ & $\begin{array}{r}634 . \\
43 . \\
1421 . \\
1622 . \\
411 .\end{array}$ & $\begin{array}{l}.600 \\
.571 \\
.571 \\
.600 \\
.600\end{array}$ & $\begin{array}{l}2326 . \\
1901 . \\
1602 . \\
2347 . \\
2249 .\end{array}$ \\
\hline 3 & $\begin{array}{l}319 . \\
320 \\
321 . \\
322 . \\
323 .\end{array}$ & $\begin{array}{r}11.230 \\
2.817 \\
3.821 \\
8.200 \\
3.085\end{array}$ & $\begin{array}{l}42+140 \\
26.260 \\
20.120 \\
31.780 \\
17.350\end{array}$ & $\begin{array}{r}2058 \\
43 \\
1421 . \\
1646 . \\
2310\end{array}$ & $\begin{array}{l}.600 \\
.571 \\
.571 \\
.600 \\
.571\end{array}$ & $\begin{array}{l}1618 \\
1856 \\
1604 \\
2347 \\
1336 .\end{array}$ \\
\hline
\end{tabular}


Table 3. Summary of 1984 data: Raft station wind speed--(1) dally average wind speed at 1,2 and 3 meters above lake surface, (2) daily maximum and minimum wind speed at the level and the time they occurred. (continued)

\begin{tabular}{|c|c|c|c|c|c|c|}
\hline $\begin{array}{c}\text { HEIGHT OF } \\
\text { MEASUREMENT } \\
\text { ABOUE LAKE } \\
\text { SURFACE } \\
(m)\end{array}$ & $\begin{array}{l}\text { JUL. I AN } \\
\text { IIAY }\end{array}$ & $\begin{array}{l}\text { IIAILY } \\
\text { AUEFAGE } \\
\text { WINI } \\
\text { SFEEII } \\
(m i / h)\end{array}$ & $\begin{array}{l}\text { IIAILY } \\
\text { MAXIMUM } \\
\text { WINII } \\
\text { SFEE[ } \\
\left(m_{1} / h\right)\end{array}$ & $\begin{array}{l}\text { TIME OF } \\
\text { MAXIMUM } \\
\text { WINI } \\
\text { SFEEI } \\
\text { (h) }\end{array}$ & $\begin{array}{l}\text { [IAILY } \\
\text { MINIMUM } \\
\text { WINII } \\
\text { SFEEI } \\
(m i / h)\end{array}$ & $\begin{array}{l}\text { TIME OF } \\
\text { MINIMUM } \\
\text { WINII } \\
\text { SFEEII } \\
\text { (h) }\end{array}$ \\
\hline 1 & $\begin{array}{l}324 . \\
325 . \\
326 . \\
327 . \\
328 . \\
329 . \\
330 . \\
331 .\end{array}$ & $\begin{array}{l}7.480 \\
9.010 \\
8.080 \\
3.464 \\
3.203 \\
4.474 \\
1.259 \\
2.231\end{array}$ & $\begin{array}{r}25.440 \\
25.950 \\
29.800 \\
15.110 \\
17.740 \\
15.250 \\
5.071 \\
17.430\end{array}$ & $\begin{array}{r}1309 . \\
31 . \\
920 . \\
1251 . \\
1319 . \\
36 . \\
1236 . \\
1148 .\end{array}$ & $\begin{array}{l}.600 \\
.600 \\
.571 \\
.571 \\
.600 \\
.600 \\
.571 \\
.571\end{array}$ & $\begin{array}{r}2013 . \\
2032 . \\
1124 . \\
422 . \\
2350 . \\
2400 . \\
821 . \\
948 .\end{array}$ \\
\hline 2 & $\begin{array}{l}324 . \\
325 . \\
326 . \\
327 . \\
328 . \\
329 . \\
330 . \\
331 .\end{array}$ & $\begin{array}{l}7.887 \\
9.430 \\
8.570 \\
3.614 \\
3.333 \\
4.656 \\
1.505 \\
2.529\end{array}$ & $\begin{array}{r}24.340 \\
26.490 \\
35.260 \\
15.310 \\
18.510 \\
16.640 \\
5.128 \\
17.690\end{array}$ & $\begin{array}{r}1059 . \\
1041 . \\
34 . \\
1251 . \\
1319 . \\
237 . \\
1134 . \\
1148 .\end{array}$ & $\begin{array}{l}.600 \\
.600 \\
.571 \\
.571 \\
.600 \\
.600 \\
.571 \\
.600\end{array}$ & $\begin{array}{r}1611 . \\
500 . \\
318 . \\
455 . \\
2350 . \\
2400 . \\
1742 . \\
2357 .\end{array}$ \\
\hline 3 & $\begin{array}{l}324 . \\
325 . \\
326 . \\
327 . \\
328 . \\
329 . \\
330 . \\
331 .\end{array}$ & $\begin{array}{l}8.150 \\
9.720 \\
8.850 \\
3.677 \\
3.380 \\
4.758 \\
1.471 \\
2.532\end{array}$ & $\begin{array}{r}25.580 \\
26.040 \\
32.120 \\
14.720 \\
19.240 \\
17.210 \\
5.495 \\
20.010\end{array}$ & $\begin{array}{r}1059 . \\
31 . \\
458 . \\
1251 . \\
1319 . \\
237 . \\
1119 . \\
1144 .\end{array}$ & $\begin{array}{l}.600 \\
.000 \\
.600 \\
.571 \\
.600 \\
.571 \\
.571 \\
.571\end{array}$ & $\begin{array}{r}958 . \\
500 . \\
2347 . \\
455 . \\
2350 . \\
1555 . \\
2030 . \\
2400 .\end{array}$ \\
\hline
\end{tabular}

\begin{tabular}{|c|c|c|c|c|c|c|}
\hline HEIGHT OF & & IIAILY & IIAILY & TIME OF & IIAILY & TIME OF \\
\hline $\begin{array}{l}\text { MEASUREMENT } \\
\text { ABOUE LAKE } \\
\text { SURFACE }\end{array}$ & $\begin{array}{l}\text { JUL. I AN } \\
\text { IIAY }\end{array}$ & $\begin{array}{l}\text { AUERAGE: } \\
\text { WINII } \\
\text { SFEEII }\end{array}$ & $\begin{array}{l}\text { MAXIMUM } \\
\text { WINII } \\
\text { SFEEI }\end{array}$ & $\begin{array}{l}\text { MAXIMUM } \\
\text { WINII } \\
\text { SFEEII }\end{array}$ & $\begin{array}{l}\text { MINIMUM } \\
\text { WINII } \\
\text { SFEEII }\end{array}$ & $\begin{array}{l}\text { MINIMUM } \\
\text { WINI } \\
\text { SFEEII }\end{array}$ \\
\hline$\left(m_{1}\right)$ & & $\left(m_{3} / h\right)$ & $(n+3 / h)$ & $(h)$ & $\left(m_{1} / h\right)$ & $(n)$ \\
\hline 1 & $\begin{array}{l}332 . \\
333 . \\
334 . \\
335 .\end{array}$ & $\begin{array}{r}.863 \\
1.112 \\
3.523 \\
3.906\end{array}$ & $\begin{array}{r}3.713 \\
8.090 \\
18.930 \\
19.530\end{array}$ & $\begin{array}{l}1326 . \\
1047 . \\
2048 . \\
559 .\end{array}$ & $\begin{array}{r}.571 \\
.571 \\
.571 \\
.543\end{array}$ & $\begin{array}{l}2341 . \\
1242 . \\
1725 . \\
214 .\end{array}$ \\
\hline 2 & $\begin{array}{l}332 . \\
333 . \\
334 . \\
335 .\end{array}$ & $\begin{array}{l}1.069 \\
1.369 \\
3.834 \\
2.823\end{array}$ & $\begin{array}{r}3.882 \\
8.970 \\
21.960 \\
20.740\end{array}$ & $\begin{array}{l}1327 . \\
1932 . \\
2137 . \\
1040 .\end{array}$ & $\begin{array}{l}.571 \\
.571 \\
.571 \\
.288\end{array}$ & $\begin{array}{r}2340 . \\
1245 . \\
1725 . \\
548 .\end{array}$ \\
\hline 3 & $\begin{array}{l}332 . \\
333 . \\
334\end{array}$ & $\begin{array}{r}.862 \\
1.335 \\
3.957\end{array}$ & $\begin{array}{r}4.392 \\
9.750 \\
22.220\end{array}$ & $\begin{array}{l}1328 . \\
1932 . \\
2040 .\end{array}$ & $\begin{array}{r}.571 \\
.571 \\
.571\end{array}$ & $\begin{array}{l}2341 . \\
1318 \\
1725 .\end{array}$ \\
\hline & 335. & 4.787 & 23.180 & 1040 & .571 & 2341 . \\
\hline
\end{tabular}



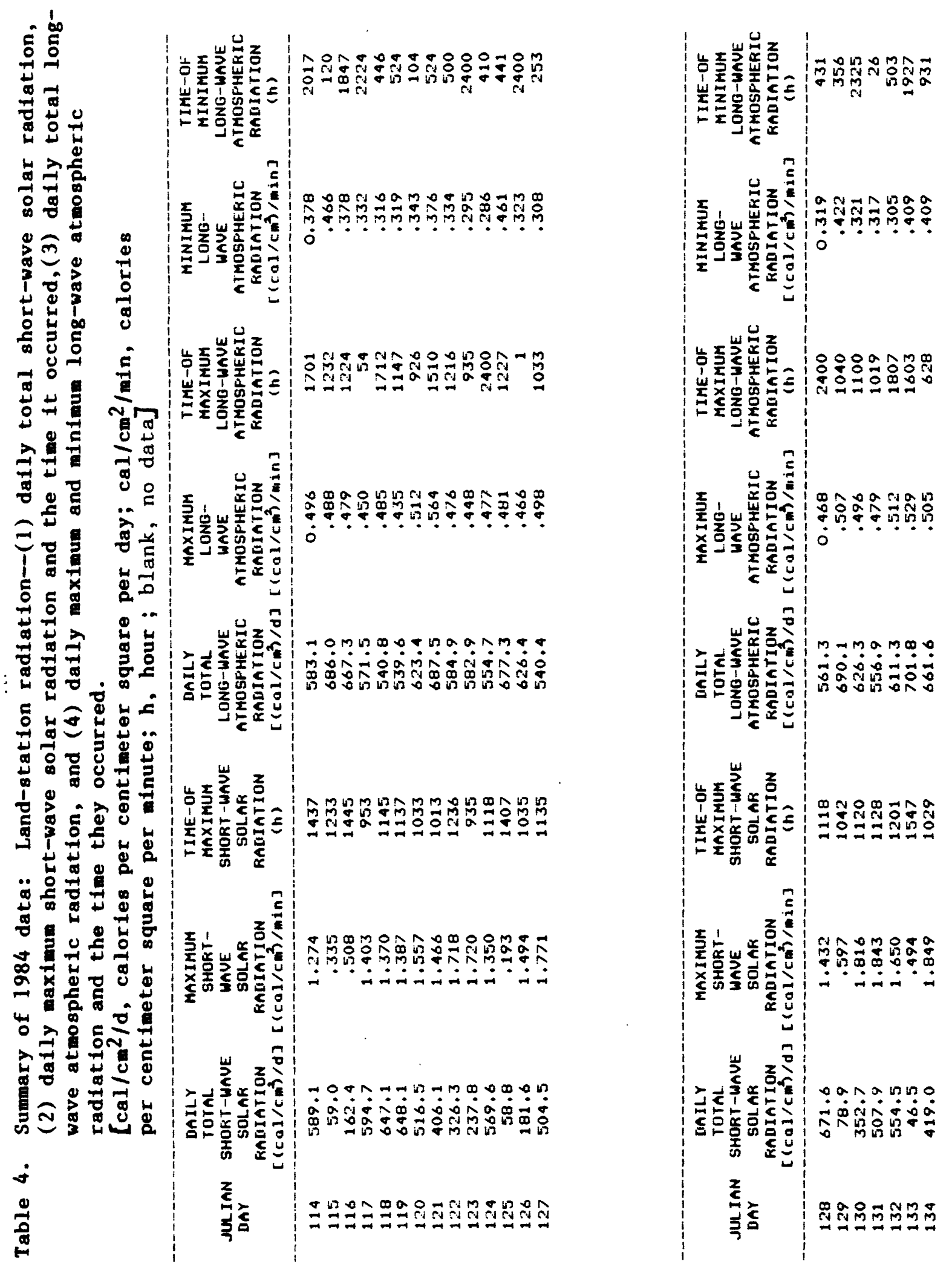

(59) 

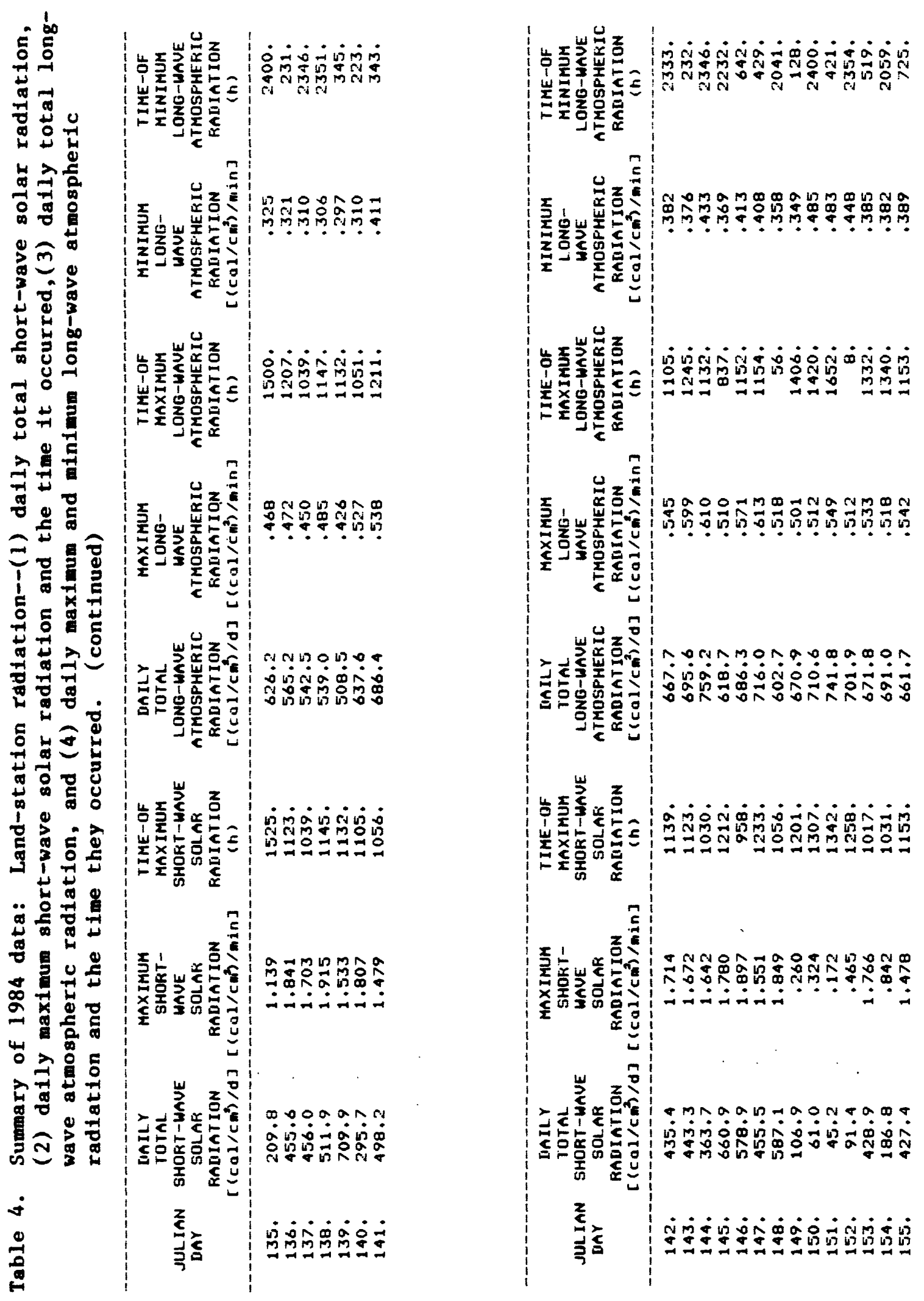

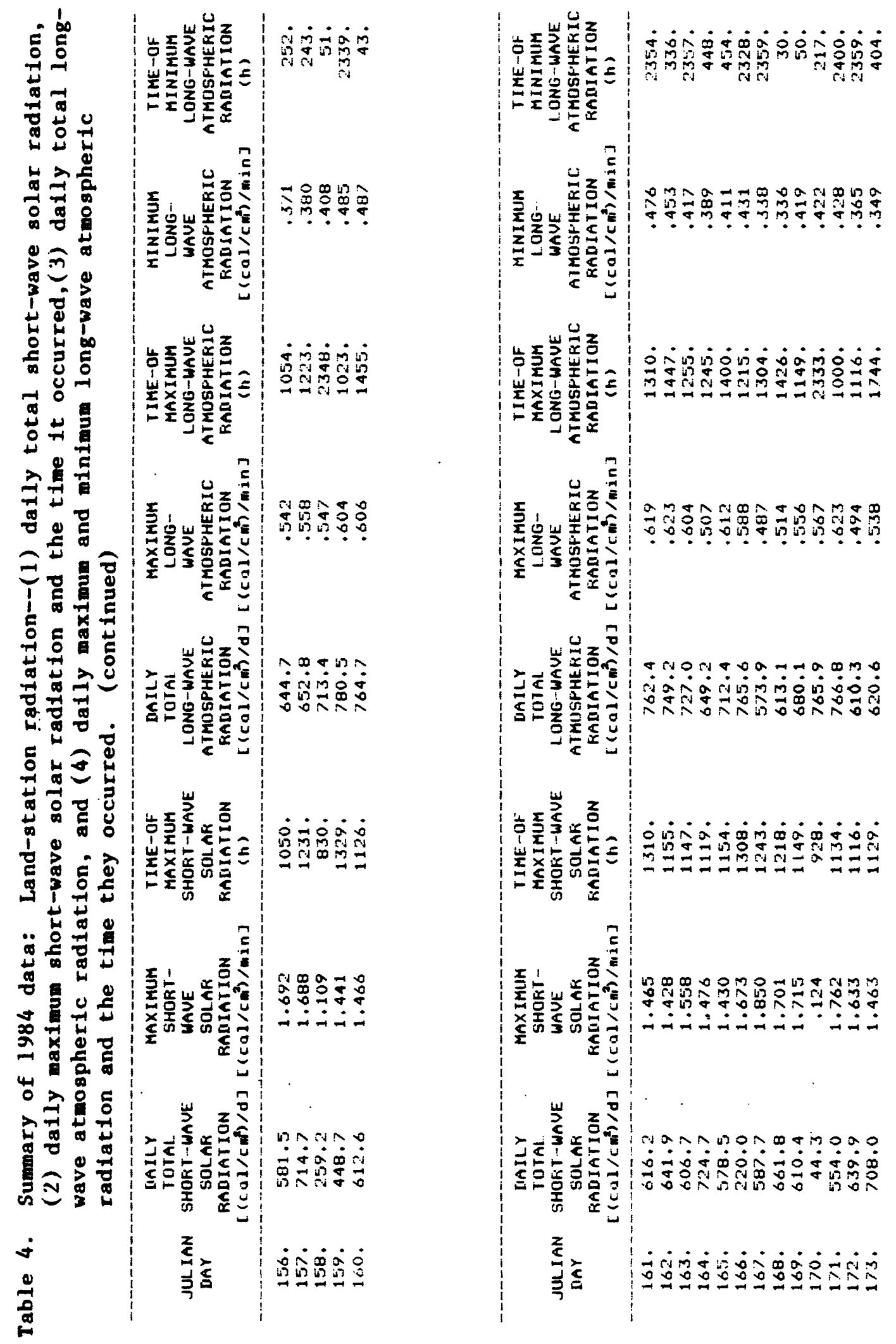

(61) 

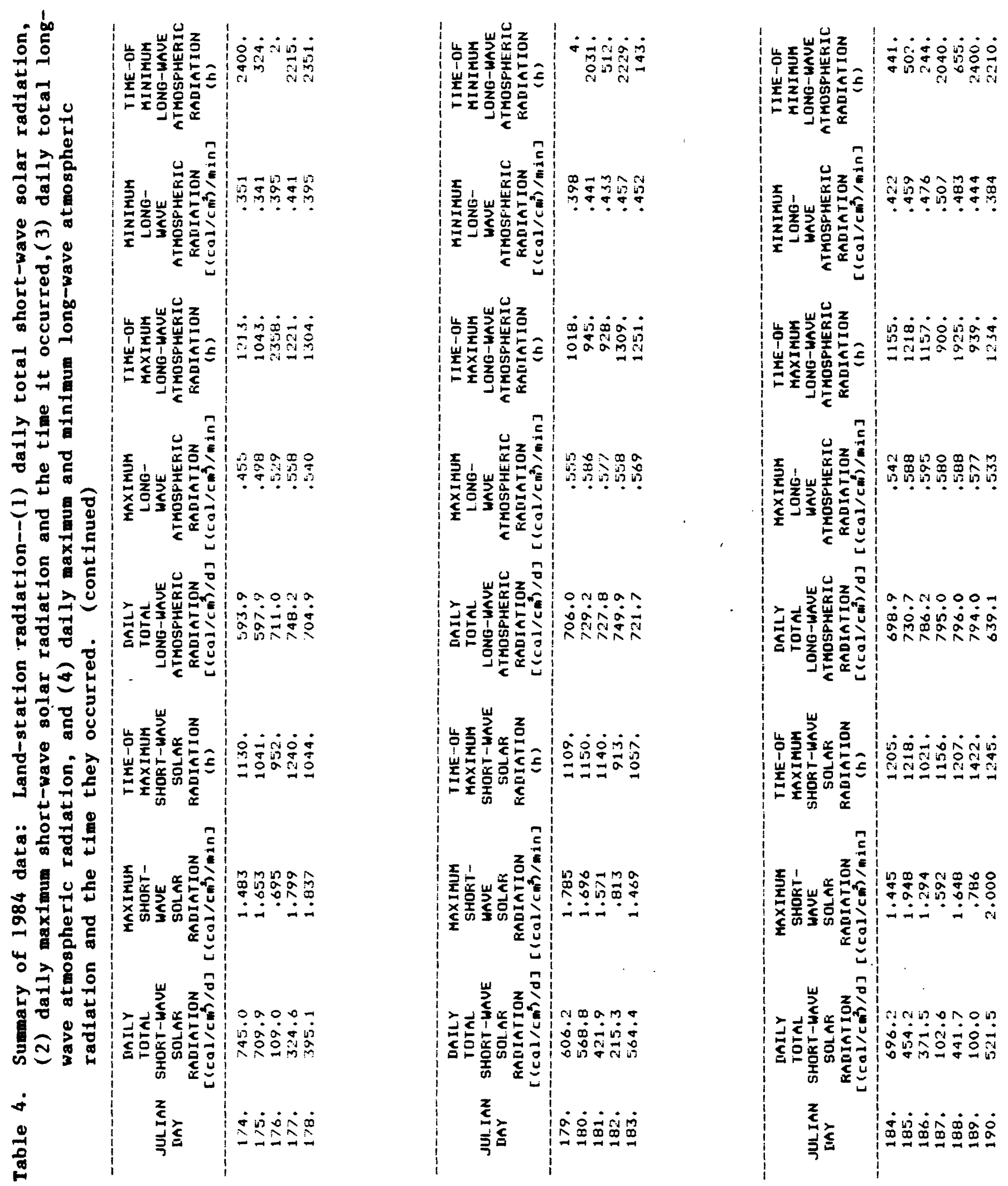

(62) 

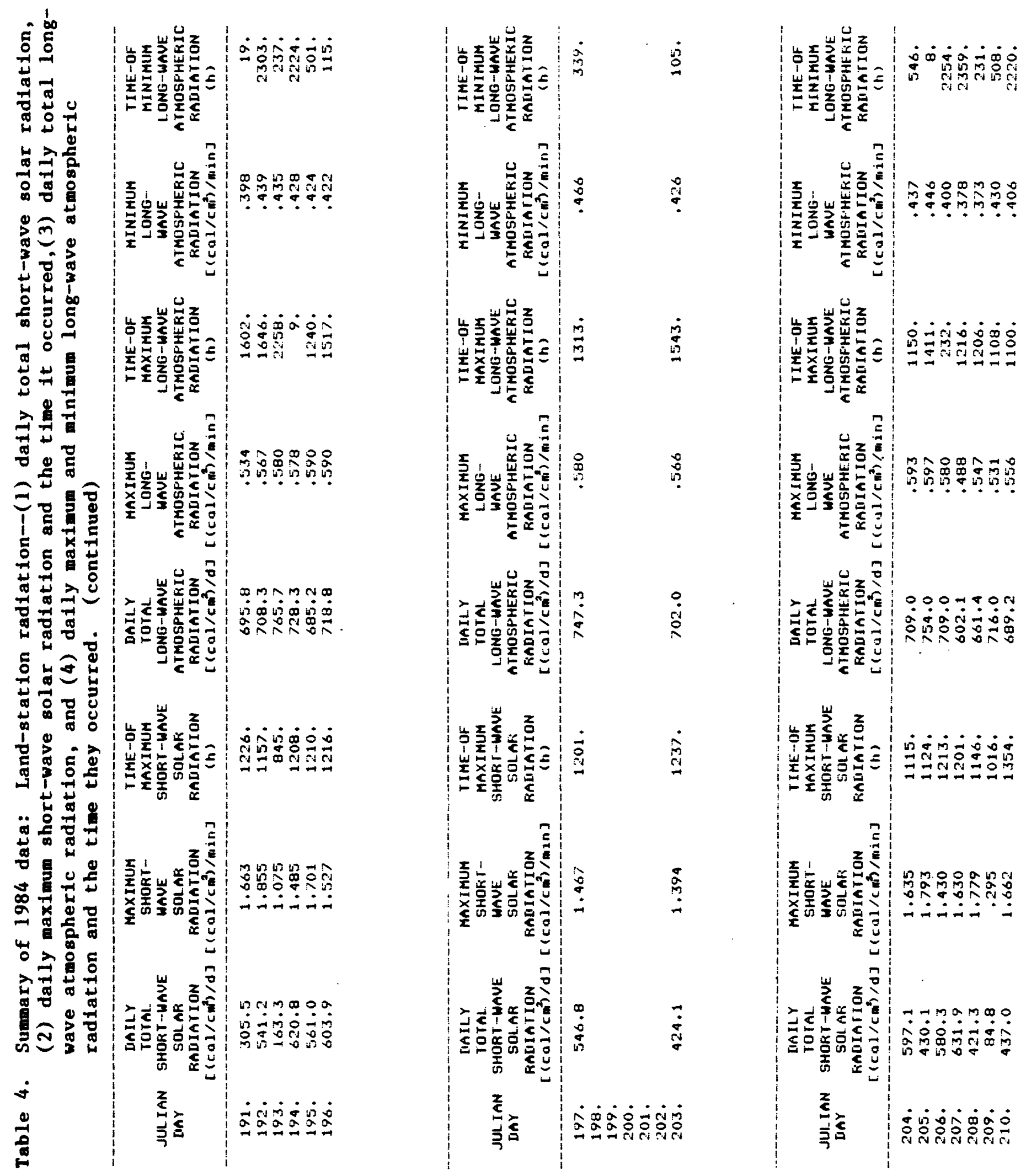

(63) 


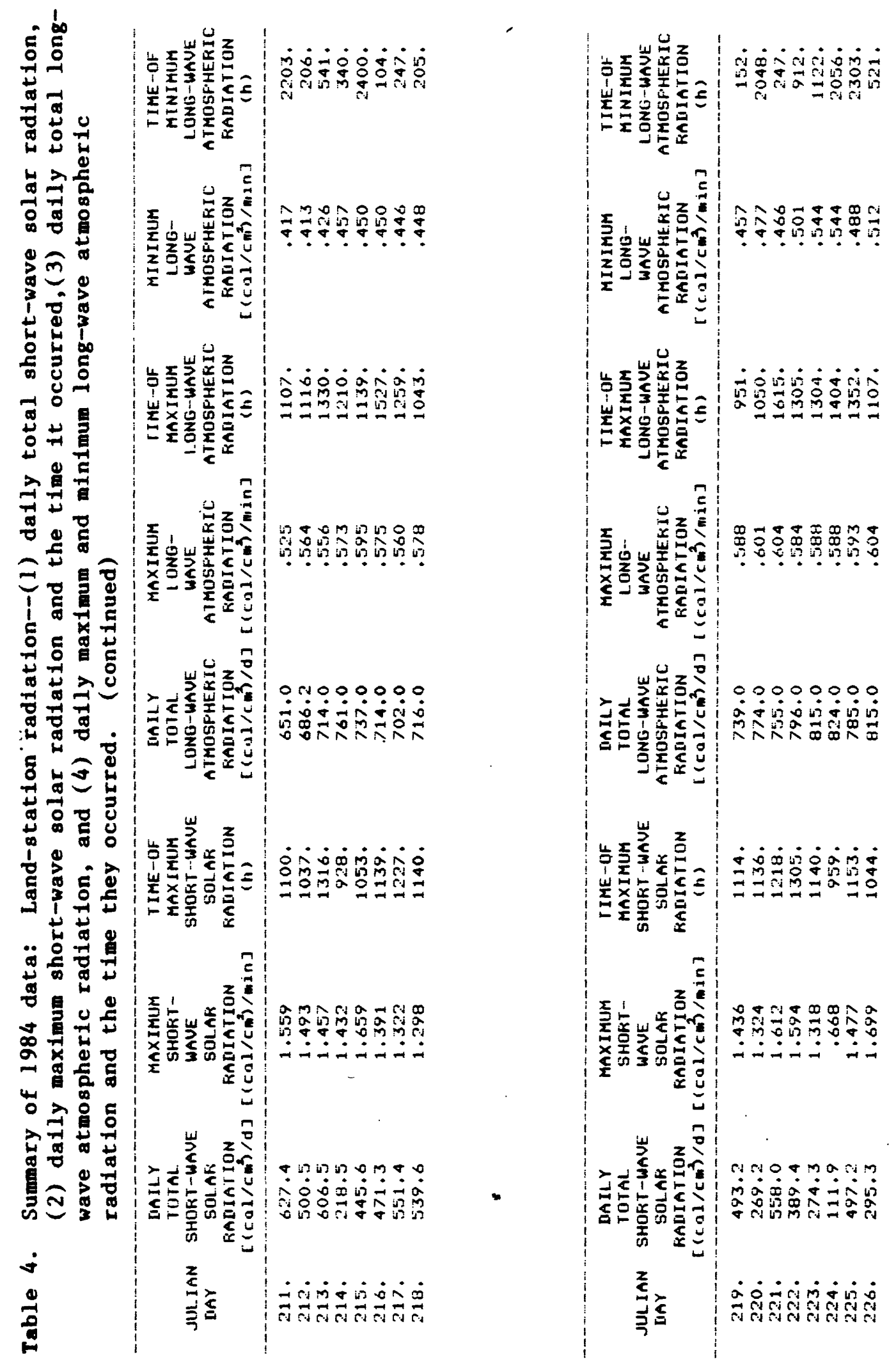



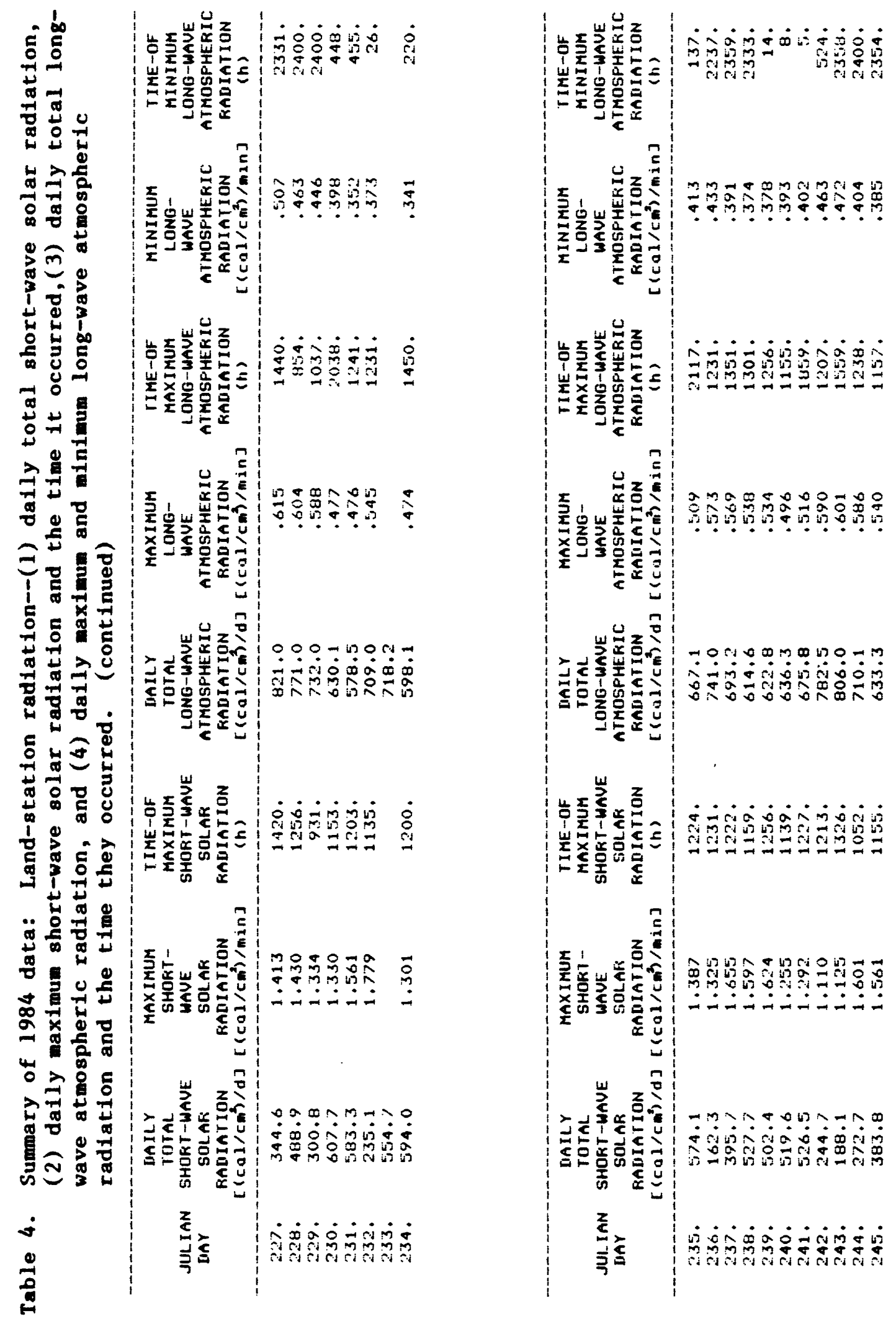

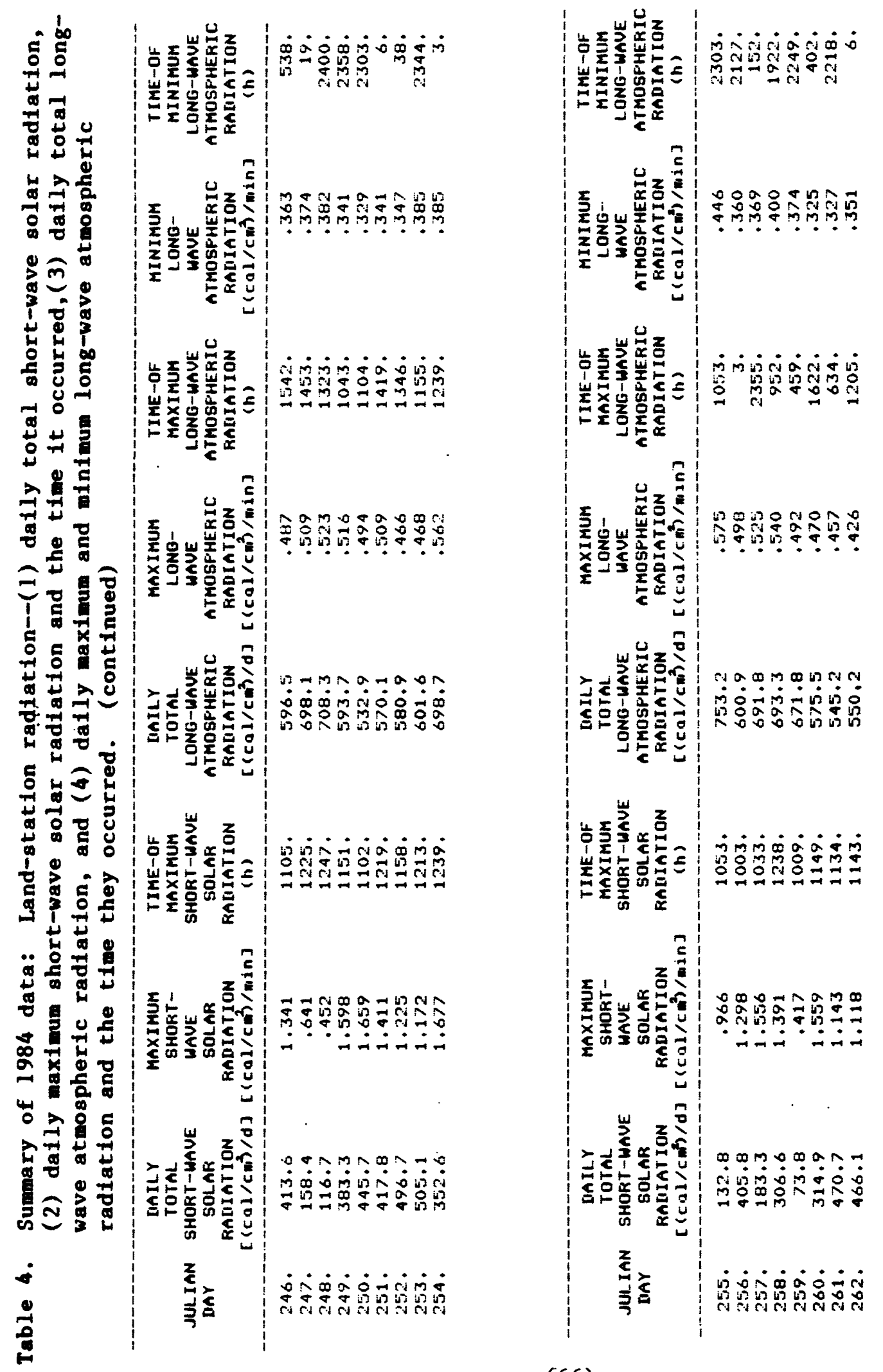

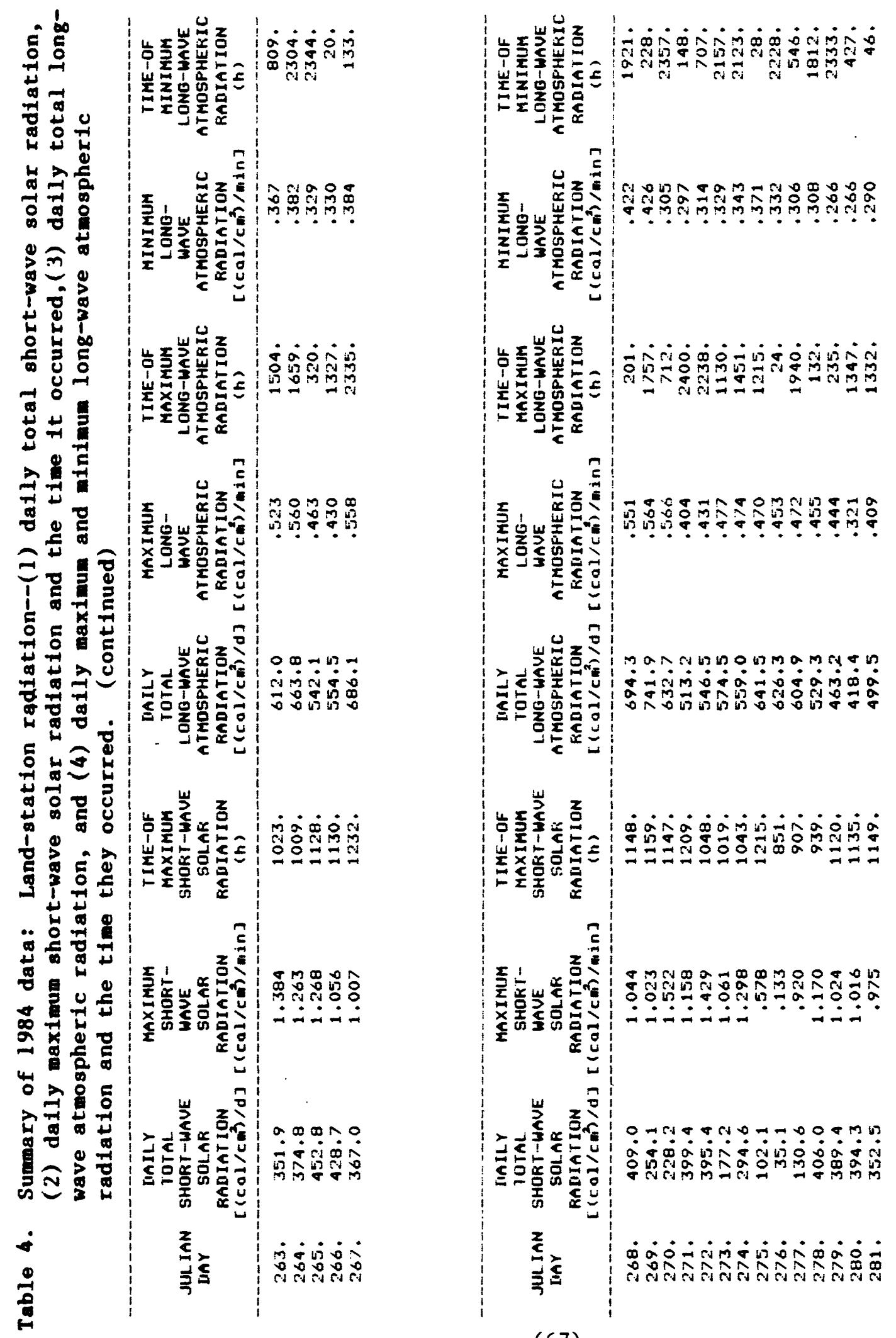

(67) 

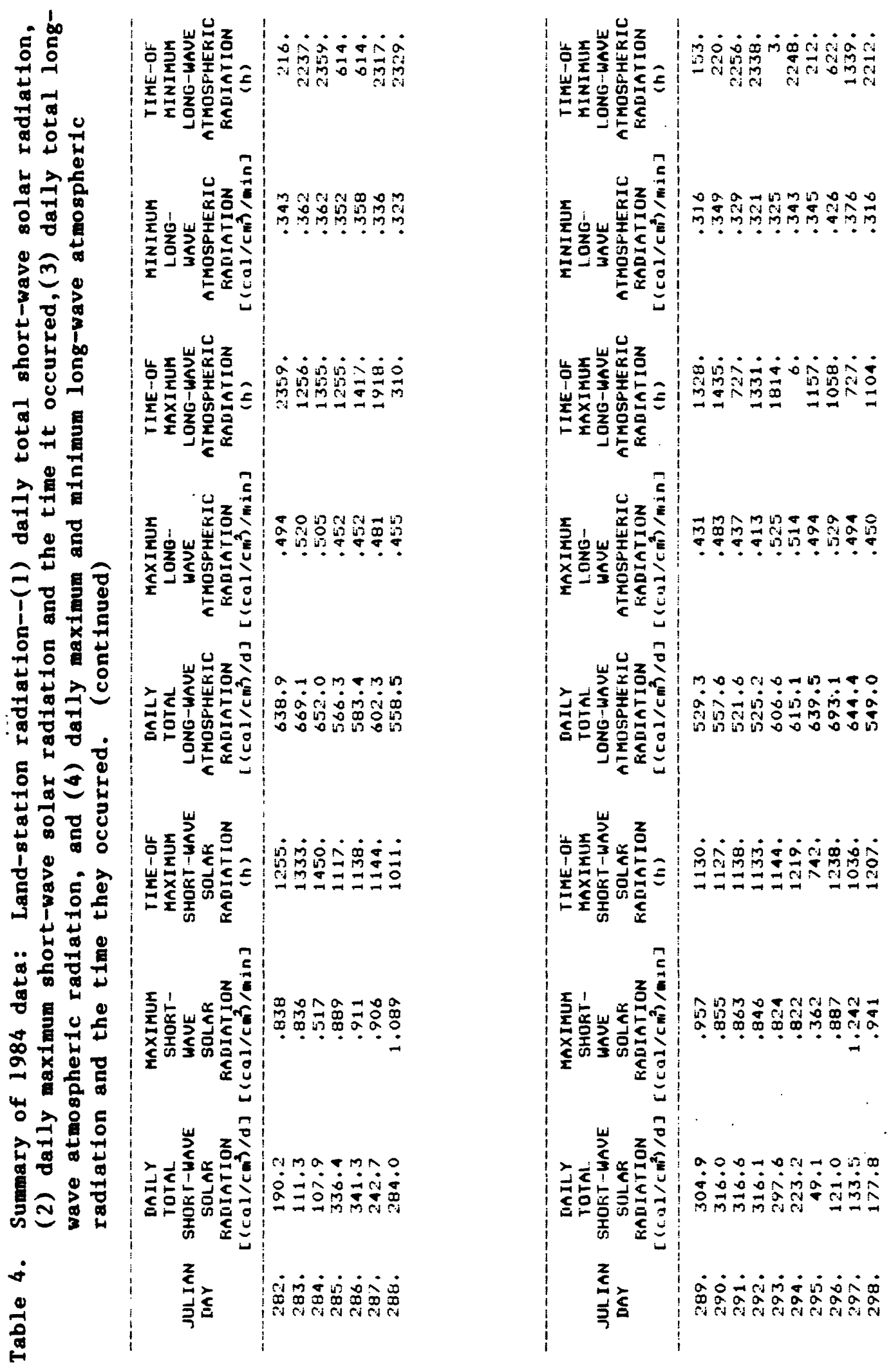

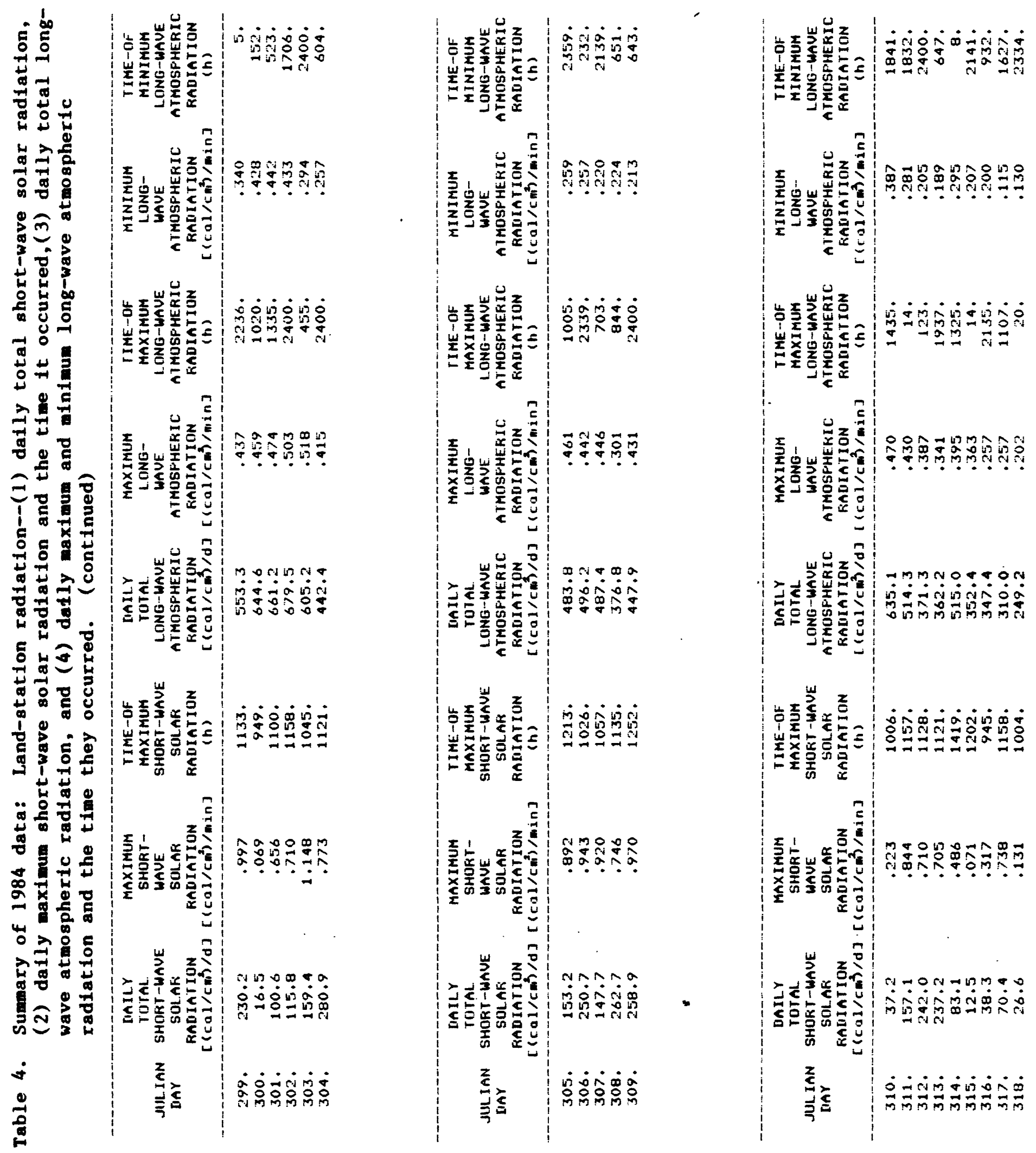

(69) 